\title{
Vertex operator algebras and the Verlinde conjecture
}

\author{
Yi-Zhi Huang
}

\begin{abstract}
We prove the Verlinde conjecture in the following general form: Let $V$ be a simple vertex operator algebra satisfying the following conditions: (i) $V_{(n)}=0$ for $n<0, V_{(0)}=\mathbb{C} 1$ and $V^{\prime}$ is isomorphic to $V$ as a $V$-module. (ii) Every $\mathbb{N}$-gradable weak $V$-module is completely reducible. (iii) $V$ is $C_{2}$-cofinite. (In the presence of Condition (i), Conditions (ii) and (iii) are equivalent to a single condition, namely, that every weak $V$-module is completely reducible.) Then the matrices formed by the fusion rules among the irreducible $V$-modules are diagonalized by the matrix given by the action of the modular transformation $\tau \mapsto-1 / \tau$ on the space of characters of irreducible $V$-modules. Using this result, we obtain the Verlinde formula for the fusion rules. We also prove that the matrix associated to the modular transformation $\tau \mapsto-1 / \tau$ is symmetric.
\end{abstract}

\section{Introduction}

In the present paper, we formulate and prove a general version of the Verlinde conjecture and prove the Verlinde formula for fusion rules using the representation theory of vertex operator algebras.

The Verlinde conjecture [V] in conformal field theory states that the action of the modular transformation $\tau \mapsto-1 / \tau$ on the space of characters of a rational conformal field theory diagonalizes the fusion rules. Except for some particular examples (see below), the general Verlinde conjecture has been an open problem for twenty years. In [MS1, Moore and Seiberg showed on a physical level of rigor that this conjecture follows from the axioms for rational conformal field theories (see [K], [S1], [S2] and [S3] for axioms for conformal 
field theories and [MS2] for axioms and assumptions for rational conformal field theories on a physical level of rigor). Axioms for rational conformal field theories are in fact much stronger than statements such as the Verlinde conjecture. The work MS1] MS2 of Moore and Seiberg greatly advanced our understanding of the structure of conformal field theories. However, it is a very hard problem to actually construct theories satisfying these axioms mathematically and therefore the existence of rational conformal field theories is a very strong assumption. In fact, the construction of full rational conformal field theories, especially the hard part of verifying the axioms, need, among many other things, the Verlinde conjecture (see [HK1] and [HK2]).

Assuming that the axioms for higher-genus rational conformal field theories are satisfied, the Verlinde conjecture leads to a Verlinde formula [V] for the dimensions of the spaces of conformal blocks on higher-genus Riemann surfaces. In the special case of the conformal field theories associated to affine Lie algebras (the Wess-Zumino-Novikov-Witten models), this Verlinde formula gives a surprising formula for the dimensions of the spaces of sections of the "generalized theta divisors" and has given rise to a great deal of excitement and new mathematics. See the works [TUY] by Tsuchiya-UenoYamada, BL] by Beauville-Laszlo, [F] by Faltings and [KNR] by KumarNarasimhan-Ramanathan for details and proofs of this particular case of the Verlinde formulas.

In this paper, using the results on the duality and modular invariance of genus-zero and genus-one correlation functions, especially those obtained recently in [H6] and [H7], we formulate and prove a general version of the Verlinde conjecture in the framework of the theory of vertex operator algebras, which were first introduced and studied in mathematics by Borcherds [B] and Frenkel-Lepowsky-Meurman [FLM]. Our theorem assumes only that the vertex operator algebra we consider satisfies certain natural grading, finiteness and reductivity properties (see below). This result is part of a program to mathematically construct conformal field theories in the sense of Kontsevich [K] and Segal [S1] [S2] S3] from representations of vertex operator algebras. It will be a main step towards a mathematical proof of the Verlinde formula for the dimensions of conformal blocks on higher-genus Riemann surfaces for conformal field theories other than the Wess-Zumino-Novikov-Witten models.

To state our main result, we need to discuss briefly matrices formed by fusion rules and modular transformations. Let $V$ be a simple vertex operator algebra satisfying the following conditions: (i) $V_{(n)}=0$ for $n<0, V_{(0)}=\mathbb{C} \mathbf{1}$ 
and $V^{\prime}$ is isomorphic to $V$ as a $V$-module. (ii) Every $\mathbb{N}$-gradable weak $V$ module is completely reducible. (iii) $V$ is $C_{2}$-cofinite (that is, $\operatorname{dim} V / C_{2}(V)<$ $\infty$ where $C_{2}(V)$ is the subspace of $V$ spanned by $u_{-2} v$ for $u, v \in V$. By results of $\mathrm{Li}$ [Li] and Abe-Buhl-Dong [ABD], Conditions (ii) and (iii) are equivalent to a single condition that every weak $V$-module is completely reducible. Let $\mathcal{A}$ be the set of equivalence classes of irreducible $V$-modules. For $a \in \mathcal{A}$, we choose a representative $W^{a}$ in $a$ such that $W^{e}=V$ where $e$ is the equivalence class containing $V$.

For $a_{1}, a_{2}, a_{3} \in \mathcal{A}$, let $N_{a_{1} a_{2}}^{a_{3}}=N_{W^{a_{1}} W^{a_{2}}}^{W_{3}}$ be the corresponding fusion rules, that is, the dimensions of the spaces of intertwining operators of types $\left(\begin{array}{c}W^{a_{3}} \\ W^{a_{1}} W^{a_{2}}\end{array}\right)$ (see [FHL]). For $a \in \mathcal{A}$, let $\mathcal{N}(a)$ be the matrix whose entries are $N_{a a_{1}}^{a_{2}}$ for $a_{1}, a_{2} \in \mathcal{A}$, that is,

$$
\mathcal{N}(a)=\left(N_{a a_{1}}^{a_{2}}\right)
$$

For $a \in \mathcal{A}$, we have the characters $\operatorname{Tr}_{W^{a}} q_{\tau}^{L(0)-\frac{c}{24}}$, also called shifted graded dimensions, where $q_{\tau}=e^{2 \pi \tau}$ and $\tau \in \mathbb{H}$. In [Z], Zhu proved under certain conditions that the maps given by

$$
u \mapsto \operatorname{Tr}_{W^{a}} Y_{W^{a}}\left(e^{2 \pi z L(0)} u, e^{2 \pi z}\right) q_{\tau}^{L(0)-\frac{c}{24}}
$$

for $u \in V$, where $a \in \mathcal{A}$, are linearly independent and there exist $S_{a_{1}}^{a_{2}} \in \mathbb{C}$ for $a_{1}, a_{2} \in \mathcal{A}$ such that

$$
\begin{array}{r}
\operatorname{Tr}_{W^{a_{1}}} Y_{W^{a_{1}}}\left(e^{-\frac{2 \pi z}{\tau} L(0)}\left(-\frac{1}{\tau}\right) u, e^{-\frac{2 \pi z}{\tau}}\right) q_{-\frac{1}{\tau}}^{L(0)-\frac{c}{24}} \\
=\sum_{a_{2} \in \mathcal{A}} S_{a_{1}}^{a_{2}} \operatorname{Tr}_{W^{a_{2}}} Y_{W^{a_{2}}}\left(e^{2 \pi z L(0)} u, e^{2 \pi z}\right) q_{\tau}^{L(0)-\frac{c}{24}}
\end{array}
$$

in [DLM], Dong, Li and Mason improved Zhu's results above by showing that they also hold under the conditions (slightly weaker than what) we assume in our paper. In partiuclar, when $u=\mathbf{1}$, we have

$$
\operatorname{Tr}_{W^{a_{1}}} q_{-\frac{1}{\tau}}^{L(0)-\frac{c}{24}}=\sum_{a_{2} \in \mathcal{A}} S_{a_{1}}^{a_{2}} \operatorname{Tr}_{W^{a_{2}}} q_{\tau}^{L(0)-\frac{c}{24}}
$$

Now we can state the main result of the present paper: The matrix $S=\left(S_{a_{1}}^{a_{2}}\right)$ diagonalizes $\mathcal{N}(a)$ for $a \in \mathcal{A}$. See Theorem 5.2 for a more complete and precise statement. Using this result, we obtain the Verlinde formula for 
the fusion rules for such a vertex operator algebra. We also prove that the matrix $S$ is symmetric.

Note that fusion rules and modular transformations of characters were already defined mathematically for suitable vertex operator algebras by FrenkelHuang-Lepowsky in [FHL] using intertwining operators and by Zhu in [Z] using his modular invariance theorem, respectively. The $C_{2}$-cofiniteness condition was also introduced in [Z]. These are already enough for the mathematical formulation of the general version of the Verlinde conjecture proved in this paper. Further results on intertwining operators and modular invariance were obtained in [HL1]-HL4 by Huang-Lepowsky, in [H1, [H2] and [H5] by the author, in [DLM] by Dong-Li-Mason and in [M] by Miyamoto. However, the proof of the Verlinde conjecture in the present paper requires much stronger results than these. We need the duality and modular invariance properties for genus-zero and genus-one multi-point correlation functions constructed from intertwining operators for a vertex operator algebra satisfying the conditions above. These properties have been proved recently in [H6] and [H7] by the author so that the proof of the Verlinde conjecture in the present paper has now become possible.

The main content of the present paper is to establish mathematically certain formulas needed in the proof of the Verlinde conjecture. Most of the formulas were first obtained on a physical level of rigor by Moore and Seiberg [MS1] [MS2] using the (assumed) axioms for rational conformal field theories. In the present paper, our formulations and proofs are based on the results obtained in the theory of vertex operator algebras. We use only those results which have been established mathematically and we do not assume that all axioms for rational conformal field theories hold. In [MS1] and [MS2], Moore and Seiberg discussed many of the subtle technical details using examples such as the minimal models. Many of these discussions cannot be generalized to general cases. For example, in [MS1] and [MS2], spaces of chiral vertex operators (intertwining operators) are identified with tensor products of spaces of lowest weight vectors in modules. This identification allows them to give an $S_{3}$ action easily on the direct sum of these spaces and the formulas obtained in [MS1] and [MS2] depend heavily on this action. It is known that in general spaces of intertwining operators cannot be identified with tensor products of spaces of lowest weight vectors and thus, even if we assume that all axioms for rational conformal field theories hold, the method based on particular examples in [MS1] and [MS2] cannot be directly adopted to establish the formulas we need. In the present paper, we define 
this action of $S_{3}$ using the skew-symmetry for intertwining operators and the contragredient intertwining operators in [FHL] and [HL2] and prove all the results and formulas needed. There are other examples similar to this one in the present paper. In this sense, even if we assume that all axioms for rational conformal field theories hold, the formulas and results stated in [MS1] and MS2] are actually conjectures and in the present paper we give mathematical proofs.

The results of the present paper have been used in [H10] to prove the rigidity and modularity of the tensor category of modules for a vertex operator algebra satisfying the conditions mentioned above. They have also been used in the constructions of genus-zero and genus-one full conformal field theories in [HK1] and [HK2]. The results of the present paper have been announced in [H8] and [H9]. See also [Le] for an exposition.

We assume that the reader is familiar with the basic theory of vertex operator algebras as presented in [FLM], FHL] and [LL]. We also assume that the reader has some basic knowledge in the theories of intertwining operators, tensor products, composition-invertible formal series and the Virasoro algebra, and the modular invariance, as developed in, for example, [DLM], [HL1]-[HL4], [H1]-[H7], [M] and [Z].

The present paper is organized as follows: In Section 1, we state our basic assumptions and we discuss intertwining operators and genus-zero correlation functions constructed from them. An action of $S_{3}$ on the direct sum of spaces of intertwining operators among irreducible modules are also given in this section. In section 2, we discuss geometrically-modified intertwining operators and genus-one correlation functions constructed from these operators. The modular transformation associated to $\tau \mapsto-1 / \tau$ is recalled in this section. Section 3 is devoted to the proof of three formulas for braiding and fusing matrices. Using all the results obtained in Sections 1, 2 and 3, we prove two formulas derived first by Moore and Seiberg from the axioms for rational conformal field theories in Section 4. Finally in Section 5, we prove the Verlinde conjecture, the Verlinde formula for fusion rules and that the matrix associated to the modular transformation $\tau \mapsto-1 / \tau$ is symmetric.

Notations In this paper, $i$ is either $\sqrt{-1}$ or an index, and it should be easy to tell which is which. The symbols $\mathbb{N}, \mathbb{Z}_{+}, \mathbb{Z}, \mathbb{Q}, \mathbb{C}, \mathbb{C}^{\times}$and $\mathbb{H}$ denote the nonnegative integers, positive integers, integers, rational numbers, complex numbers, nonzero complex numbers and the upper half plane, respectively. 
We shall use $x, y, \ldots$ to denote commuting formal variables and $z, z_{1}, z_{2}, \ldots$ to denote complex numbers or complex variables.

Acknowledgment This research is supported in part by NSF grants DMS0070800 and DMS-0401302. I am grateful to J. Lepowsky, L. Kong and the referee for their comments.

\section{Intertwining operators and genus-zero cor- relation functions}

Let $V$ be a simple vertex operator algebra and $C_{2}(V)$ the subspace of $V$ spanned by $u_{-2} v$ for $u, v \in V$. In the present paper, we shall always assume that $V$ satisfies the following conditions:

1. $V_{(n)}=0$ for $n<0, V_{(0)}=\mathbb{C} 1$ and $V^{\prime}$ is isomorphic to $V$ as a $V$-module.

2. Every $\mathbb{N}$-gradable weak $V$-module is completely reducible.

3. $V$ is $C_{2}$-cofinite, that is, $\operatorname{dim} V / C_{2}(V)<\infty$.

Note that when $V_{(n)}=0$ for $n<0, V_{(0)}=\mathbb{C} \mathbf{1}, V^{\prime}$ is isomorphic to $V$ as a $V$-module if for any irreducible $V$-module not isomorphic to $V, W_{(0)}=0$. So the results of the present paper still hold if Condition 1 above is replaced by the condition $V_{(n)}=0$ for $n<0, V_{(0)}=\mathbb{C} \mathbf{1}$, and $W_{(0)}=0$ for any irreducible $V$-module not isomorphic to $V$. As we have mentioned in the abstract and introduction, by results of Li [Li] and Abe-Buhl-Dong [ABD], Conditions (ii) and (iii) are equivalent to a single condition that every weak $V$-module is completely reducible.

From [DLM], we know that there are only finitely many inequivalent irreducible $V$-modules. Let $\mathcal{A}$ be the set of equivalence classes of irreducible $V$-modules. We denote the equivalence class containing $V$ by $e$. Note that the contragredient module of an irreducible module is also irreducible (see [FHL]). So we have a map

$$
\begin{aligned}
\prime: \mathcal{A} & \rightarrow \mathcal{A} \\
a & \mapsto a^{\prime} .
\end{aligned}
$$

For $a \in \mathcal{A}$, if $a \neq a^{\prime}$, we choose representatives $W^{a}$ and $W^{a^{\prime}}$ of $a$ and $a^{\prime}$ such that $\left(W^{a}\right)^{\prime}=W^{a^{\prime}}$ and, after we identify $\left(W^{a}\right)^{\prime \prime}$ with $W^{a}$, we also 
have $\left(W^{a^{\prime}}\right)^{\prime}=W^{a}$. If $a=a^{\prime} \neq e$, we choose any nondegenerate symmetric bilinear invariant form on $W^{a}$ and using this form, we can identify $\left(W^{a}\right)^{\prime}$ with $W^{a^{\prime}}$ (and $\left(W^{a^{\prime}}\right)^{\prime}$ with $\left.W^{a}\right)$. For $a=e$, we choose the nondegenerate symmetric bilinear invariant form $(\cdot, \cdot)$ normalized by $(\mathbf{1}, \mathbf{1})=1$. Since the results of the present paper involve only elements of $\mathcal{A}$, not representatives of these elements, it is convenient to identify $V$-modules and their double contragredient modules and to identify $\left(W^{a}\right)^{\prime}$ with $W^{a^{\prime}}$ and $\left(W^{a^{\prime}}\right)^{\prime}$ with $W^{a}$ using the chosen nondegenerate bilinear invariant forms. After these identifications, we see that we can find a representative $W^{a}$ of $a$ for each $a \in \mathcal{A}$ such that $W^{e}=V$ and $\left(W^{a}\right)^{\prime}=W^{a^{\prime}}$. In this paper, for simplicity, we fix such a choice. From [AM] and [DLM], we know that irreducible $V$ modules are in fact graded by rational numbers. Thus for $a \in \mathcal{A}$, there exists $h_{a} \in \mathbb{Q}$ such that $W^{a}=\coprod_{n \in h_{a}+\mathbb{N}} W_{(n)}$ and $W_{\left(h_{a}\right)} \neq 0$.

For $a_{1}, a_{2}, a_{3} \in \mathcal{A}$, let $\mathcal{V}_{a_{1} a_{2}}^{a_{3}}$ be the space of intertwining operators of type $\left(\begin{array}{c}W^{a_{3}} \\ W^{a_{1}} W^{a_{2}}\end{array}\right)$. For any $\mathcal{Y} \in \mathcal{V}_{a_{1} a_{2}}^{a_{3}}$, we know from [FHL that for $w_{a_{1}} \in W^{a_{1}}$ and $w_{a_{2}} \in W^{a_{2}}$

$$
\mathcal{Y}\left(w_{a_{1}}, x\right) w_{a_{2}} \in x^{\Delta(\mathcal{Y})} W^{a_{3}}\left[\left[x, x^{-1}\right]\right]
$$

where

$$
\Delta(\mathcal{Y})=h_{a_{3}}-h_{a_{1}}-h_{a_{2}} .
$$

In the present paper, we shall use the following conventions: For $z \in \mathbb{C}^{\times}$, $\log z=\log |z|+i \arg z$, where $0 \leq \arg z<2 \pi$. For $z \in \mathbb{C}^{\times}$and $r \in \mathbb{C}$, $z^{r}=e^{r \log z}$. For $n \in \mathbb{Z}$ and $s \in \mathbb{C},\left(e^{n \pi i}\right)^{s}=e^{s n \pi i}$. Similarly, for $z \in \mathbb{C}^{\times}$and $O$ an linear operator, $z^{O}=e^{(\log z) O}$, if $e^{(\log z) O}$ is well-defined. For $n \in \mathbb{Z}$ and $O$ a linear operator, $\left(e^{n \pi i}\right)^{O}=e^{n \pi i O}$, if $e^{n \pi i O}$ is well defined. For $a_{1}, a_{2}, a_{3} \in \mathcal{A}$, $z \in \mathbb{C}^{\times}, r \in \mathbb{C}, \mathcal{Y} \in \mathcal{V}_{a_{1} a_{2}}^{a_{3}}, w_{a_{1}} \in W^{a_{1}}$ and $w_{a_{2}} \in W^{a_{2}}, \mathcal{Y}\left(w_{a_{1}}, z\right) w_{a_{2}}$ is $\left.\mathcal{Y}\left(w_{a_{1}}, x\right) w_{a_{2}}\right|_{x^{n}=e^{n \log z}, n \in \mathbb{C}}$ and $\mathcal{Y}\left(w_{a_{1}}, e^{r} x\right) w_{a_{2}}$ is $\left.\mathcal{Y}\left(w_{a_{1}}, y\right) w_{a_{2}}\right|_{y^{n}=e^{n r} x^{n}, n \in \mathbb{C}}$ by the conventions above.

We also know that the fusion rules $N_{a_{1} a_{2}}^{a_{3}}=N_{W^{a_{1} W^{a_{2}}}}^{a_{3}}$ for $a_{1}, a_{2}, a_{3} \in \mathcal{A}$ are all finite (see [GN], [Li], [AN], [H6]). For $a_{1}, a_{2}, a_{3} \in \mathcal{A}$, we have isomorphisms $\Omega_{-r}: \mathcal{V}_{a_{1} a_{2}}^{a_{3}} \rightarrow \mathcal{V}_{a_{2} a_{1}}^{a_{3}}$ and $A_{-r}: \mathcal{V}_{a_{1} a_{2}}^{a_{3}} \rightarrow \mathcal{V}_{a_{1} a_{3}^{\prime}}^{a_{2}^{\prime}}$ for $r \in \mathbb{Z}$, defined in Section 7 of [HL2]. Using these isomorphisms, we define a left action of the symmetric group $S_{3}$ on

$$
\mathcal{V}=\coprod_{a_{1}, a_{2}, a_{3} \in \mathcal{A}} \mathcal{V}_{a_{1} a_{2}}^{a_{3}}
$$

as follows: For $a_{1}, a_{2}, a_{3} \in \mathcal{A}, \mathcal{Y} \in \mathcal{V}_{a_{1} a_{2}}^{a_{3}}$, we define

$$
\sigma_{12}(\mathcal{Y})=e^{\pi i \Delta(\mathcal{Y})} \Omega_{-1}(\mathcal{Y})
$$




$$
\begin{aligned}
& =e^{-\pi i \Delta(\mathcal{Y})} \Omega_{0}(\mathcal{Y}) \\
\sigma_{23}(\mathcal{Y}) & =e^{\pi i h_{a_{1}}} A_{-1}(\mathcal{Y}) \\
& =e^{-\pi i h_{a_{1}}} A_{0}(\mathcal{Y}) .
\end{aligned}
$$

Proposition 1.1 The actions $\sigma_{12}$ and $\sigma_{23}$ of (12) and (23) on $\mathcal{V}$ defined above generate an action of $S_{3}$ on $\mathcal{V}$.

Proof. We need only prove the relations which must be satisfied by $\sigma_{12}$ and $\sigma_{23}$. Propositions 7.1 and 7.3 in [HL2] are actually equivalent to $\sigma_{12}^{2}=1$ and $\sigma_{23}^{2}=1$, respectively. So the only relation we need to prove is

$$
\sigma_{12} \sigma_{23} \sigma_{12} \sigma_{23}=\sigma_{23} \sigma_{12}
$$

Let $\mathcal{Y}$ be an element of $\mathcal{V}_{a_{1} a_{2}}^{a_{3}}$. Then for $w_{a_{1}} \in W^{a_{1}}, w_{a_{2}} \in W_{a_{2}}$ and $w_{a_{3}}^{\prime} \in W_{a_{3}^{\prime}}$,

$$
\begin{aligned}
& \left\langle\sigma_{23}\left(\sigma_{12}(\mathcal{Y})\right)\left(w_{a_{2}}, x\right) w_{a_{3}}^{\prime}, w_{a_{1}}\right\rangle \\
& \quad=e^{\pi i h_{a_{2}}}\left\langle w_{a_{3}}^{\prime}, \sigma_{12}(\mathcal{Y})\left(e^{x L(1)}\left(e^{-\pi i} x^{-2}\right)^{L(0)} w_{a_{2}}, x^{-1}\right) w_{a_{1}}\right\rangle \\
& \quad=e^{\pi i\left(h_{a_{3}}-h_{a_{1}}\right)}\left\langle w_{a_{3}}^{\prime}, e^{x^{-1} L(-1)} \mathcal{Y}\left(w_{a_{1}}, e^{-\pi i} x^{-1}\right) e^{x L(1)}\left(e^{-\pi i} x^{-2}\right)^{L(0)} w_{a_{2}}\right\rangle .
\end{aligned}
$$

On the other hand,

$$
\begin{aligned}
& \left\langle\sigma_{12}\left(\sigma_{23}\left(\sigma_{12}\left(\sigma_{23}(\mathcal{Y})\right)\right)\right)\left(w_{a_{2}}, x\right) w_{a_{3}}^{\prime}, w_{a_{1}}\right\rangle \\
& =e^{\pi i\left(h_{a_{1}}-h_{a_{2}}-h_{a_{3}}\right)}\left\langle e^{x L(-1)} \sigma_{23}\left(\sigma_{12}\left(\sigma_{23}(\mathcal{Y})\right)\right)\left(w_{a_{3}}^{\prime}, e^{-\pi i} x\right) w_{a_{2}}, w_{a_{1}}\right\rangle \\
& =e^{\pi i\left(h_{a_{1}}-h_{a_{2}}\right)} \text {. } \\
& \cdot\left\langle w_{a_{2}}, \sigma_{12}\left(\sigma_{23}(\mathcal{Y})\right)\left(e^{-x L(1)}\left(e^{-\pi i}\left(e^{-\pi i} x\right)^{-2}\right)^{L(0)} w_{a_{3}}^{\prime}, e^{\pi i} x^{-1}\right) e^{x L(1)} w_{a_{1}}\right\rangle \\
& =e^{-\pi i h_{a_{3}}}\left\langle w_{a_{2}}, e^{-x^{-1} L(-1)} \sigma_{23}(\mathcal{Y})\left(e^{x L(1)} w_{a_{1}}, x^{-1}\right) e^{-x L(1)}\left(e^{\pi i} x^{-2}\right)^{L(0)} w_{a_{3}}^{\prime}\right\rangle \\
& =e^{\pi i\left(h_{a_{1}}-h_{a_{3}}\right)}\left\langle\mathcal{Y}\left(e^{x^{-1} L(1)}\left(e^{-\pi i}\left(x^{-1}\right)^{-2}\right)^{L(0)} e^{x L(1)} w_{a_{1}}, x\right) e^{-x^{-1} L(1)} w_{a_{2}},\right. \\
& \left.e^{-x L(1)}\left(e^{\pi i} x^{-2}\right)^{L(0)} w_{a_{3}}^{\prime}\right\rangle \\
& =e^{\pi i\left(h_{a_{1}}-h_{a_{3}}\right)}\left\langle w_{a_{3}}^{\prime},\left(e^{\pi i} x^{-2}\right)^{L(0)} e^{-x L(-1)} .\right. \\
& \left.\cdot \mathcal{Y}\left(e^{x^{-1} L(1)}\left(e^{-\pi i} x^{2}\right)^{L(0)} e^{x L(1)} w_{a_{1}}, x\right) e^{-x^{-1} L(1)} w_{a_{2}}\right\rangle \\
& =e^{\pi i\left(h_{a_{1}}-h_{a_{3}}\right)}\left\langle w_{a_{3}}^{\prime}, e^{x^{-1} L(-1)}\left(e^{\pi i} x^{-2}\right)^{L(0)}\right. \text {. } \\
& \left.\cdot \mathcal{Y}\left(e^{x^{-1} L(1)}\left(e^{-\pi i} x^{2}\right)^{L(0)} e^{x L(1)} w_{a_{1}}, x\right) e^{-x^{-1} L(1)} w_{a_{2}}\right\rangle \\
& =e^{\pi i\left(h_{a_{1}}-h_{a_{3}}\right)}\left\langle w_{a_{3}}^{\prime}, e^{x^{-1} L(-1)}\right. \text {. }
\end{aligned}
$$




$$
\begin{array}{r}
\mathcal{Y}\left(\left(e^{\pi i} x^{-2}\right)^{L(0)} e^{x^{-1} L(1)}\left(e^{-\pi i} x^{2}\right)^{L(0)} e^{x L(1)} w_{a_{1}}, e^{\pi i} x^{-1}\right) . \\
\left.\cdot\left(e^{\pi i} x^{-2}\right)^{L(0)} e^{-x^{-1} L(1)} w_{a_{2}}\right\rangle \\
=e^{\pi i\left(h_{a_{3}}-h_{a_{1}}\right)}\left\langle w_{a_{3}}^{\prime}, e^{x^{-1} L(-1)} \mathcal{Y}\left(w_{a_{1}}, e^{-\pi i} x^{-1}\right) e^{x L(1)}\left(e^{-\pi i} x^{-2}\right)^{L(0)} w_{a_{2}}\right\rangle .
\end{array}
$$

From (1.3) and (1.4), we obtain

$$
\left\langle\sigma_{23}\left(\sigma_{12}(\mathcal{Y})\right)\left(w_{a_{2}}, x\right) w_{a_{3}}^{\prime}, w_{a_{1}}\right\rangle=\left\langle\sigma_{12}\left(\sigma_{23}\left(\sigma_{12}\left(\sigma_{23}(\mathcal{Y})\right)\right)\right)\left(w_{a_{2}}, x\right) w_{a_{3}}^{\prime}, w_{a_{1}}\right\rangle .
$$

Since $a_{1}, a_{2}, a_{3}, w_{a_{1}}, w_{a_{2}}, w_{a_{3}}^{\prime}$ and $\mathcal{Y}$ are arbitrary, we see that (1.2) holds.

For $p=1, \ldots, 6$ and $a_{1}, a_{2}, a_{3} \in \mathcal{A}$, let $\mathcal{Y}_{a_{1} a_{2} ; i}^{a_{3} ; p}, i=1, \ldots, N_{a_{1} a_{2}}^{a_{3}}$, be bases of $\mathcal{V}_{a_{1} a_{2}}^{a_{3}}$. From Thereom 3.9 in [H6], the space $\coprod_{a \in \mathcal{A}} W^{a}$ has a natural structure of an intertwining operator algebra in the sense of [H4] and [H5]. In particular, we have the associativity of intertwining operators (see (14.55) in [H1, Condition (vii) in Definition 3.1 in [H4] and Axiom 3 in Definition 2.1 in [H5]). Thus there exist

$$
F\left(\mathcal{Y}_{a_{1} a_{5} ; i}^{a_{4} ;(1)} \otimes \mathcal{Y}_{a_{2} a_{3} ; j}^{a_{5} ;(2)} ; \mathcal{Y}_{a_{6} a_{3} ; l}^{a_{4} ;(3)} \otimes \mathcal{Y}_{a_{1} a_{2} ; k}^{a_{6} ;(4)}\right) \in \mathbb{C}
$$

for $a_{1}, \ldots, a_{6} \in \mathcal{A}, i=1, \ldots, N_{a_{1} a_{5}}^{a_{4}}, j=1, \ldots, N_{a_{2} a_{3}}^{a_{5}}, k=1, \ldots, N_{a_{1} a_{2}}^{a_{6}}$, $l=1, \ldots, N_{a_{6} a_{3}}^{a_{4}}$, such that

$$
\begin{aligned}
&\left\langle w_{a_{4}^{\prime}}, \mathcal{Y}_{a_{1} a_{5} ; i}^{a_{4} ;(1)}\left(w_{a_{1}}, z_{1}\right) \mathcal{Y}_{a_{2} a_{3} ; j}^{a_{5} ;(2)}\left(w_{a_{2}}, z_{2}\right) w_{a_{3}}\right\rangle \\
&=\sum_{a_{6} \in \mathcal{A}} \sum_{k=1}^{N_{a_{6}} a_{2}} \sum_{l=1}^{N_{a_{6}}^{a_{4} a_{3}}} F\left(\mathcal{Y}_{a_{1} a_{5} ; i}^{a_{4} ;(1)} \otimes \mathcal{Y}_{a_{2} a_{3} ; j}^{a_{5} ;(2)} ; \mathcal{Y}_{a_{6} a_{3} ; l}^{a_{4} ;(3)} \otimes \mathcal{Y}_{a_{1} a_{2} ; k}^{a_{6} ;(4)}\right) . \\
& \quad \cdot\left\langle w_{a_{4}^{\prime}}, \mathcal{Y}_{a_{6} a_{3} ; l}^{a_{4} ;(3)}\left(\mathcal{Y}_{a_{1} a_{2} ; k}^{a_{6} ;(4)}\left(w_{a_{1}}, z_{1}-z_{2}\right) w_{a_{2}}, z_{2}\right) w_{a_{3}}\right\rangle
\end{aligned}
$$

when $\left|z_{1}\right|>\left|z_{2}\right|>\left|z_{1}-z_{2}\right|>0$, for $a_{1}, \ldots, a_{5} \in \mathcal{A}, w_{a_{1}} \in W^{a_{1}}, w_{a_{2}} \in W^{a_{2}}$, $w_{a_{3}} \in W^{a_{3}}, w_{a_{4}^{\prime}} \in W^{a_{4}^{\prime}}=\left(W^{a_{4}}\right)^{\prime}, i=1, \ldots, N_{a_{1} a_{5}}^{a_{4}}$ and $j=1, \ldots, N_{a_{2} a_{3}}^{a_{5}}$. Note that here we have used our convention on the choices of values of intertwining operators. The numbers

$$
F\left(\mathcal{Y}_{a_{1} a_{5} ; i}^{a_{4} ;(1)} \otimes \mathcal{Y}_{a_{2} a_{3} ; j}^{a_{5} ;(2)} ; \mathcal{Y}_{a_{6} a_{3} ; l}^{a_{4} ;(3)} \otimes \mathcal{Y}_{a_{1} a_{2} ; k}^{a_{6} ;(4)}\right)
$$

are matrix elements of the fusing isomorphism. In [H5], when these four bases are chosen to be the same, these matrix elements are denoted as $F_{a_{5} ; a_{6}}^{i, j ; k l}\left(a_{1}, a_{2}, a_{3} ; a_{4}\right)$. In the present paper, since we want to emphasize their 
dependence on the bases and since we shall need matrix elements of the fusing isomorphism under different bases, we have to instead use the notation above.

The fusing isomorphism is invertible. Thus for any bases as above, there exist

$$
F^{-1}\left(\mathcal{Y}_{a_{6} a_{3} ; l}^{a_{4} ;(1)} \otimes \mathcal{Y}_{a_{1} a_{2} ; k}^{a_{6} ;(2)} ; \mathcal{Y}_{a_{1} a_{5} ; i}^{a_{4} ;(3)} \otimes \mathcal{Y}_{a_{2} a_{3} ; j}^{a_{5} ;(4)}\right) \in \mathbb{C}
$$

for $a_{1}, \ldots, a_{6} \in \mathcal{A}, i=1, \ldots, N_{a_{1} a_{5}}^{a_{4}}, j=1, \ldots, N_{a_{2} a_{3}}^{a_{5}}, k=1, \ldots, N_{a_{1} a_{2}}^{a_{6}}$, $l=1, \ldots, N_{a_{6} a_{3}}^{a_{4}}$, such that

$$
\begin{gathered}
\left\langle w_{a_{4}^{\prime}}, \mathcal{Y}_{a_{6} a_{3} ; l}^{a_{4} ;(1)}\left(\mathcal{Y}_{a_{1} a_{2} ; k}^{a_{6} ;(2)}\left(w_{a_{1}}, z_{1}-z_{2}\right) w_{a_{2}}, z_{2}\right) w_{a_{3}}\right\rangle \\
=\sum_{a_{5} \in \mathcal{A}} \sum_{i=1}^{N_{a_{1} a_{5}}^{a_{4}}} \sum_{j=1}^{N_{a_{2} a_{3}}^{a_{2}}} F^{-1}\left(\mathcal{Y}_{a_{6} a_{3} ; l}^{a_{4} ;(1)} \otimes \mathcal{Y}_{a_{1} a_{2} ; k}^{a_{6} ;(2)} ; \mathcal{Y}_{a_{1} a_{5} ; i}^{a_{4} ;(3)} \otimes \mathcal{Y}_{a_{2} a_{3} ; j}^{a_{5} ;(4)}\right) \\
\cdot\left\langle w_{a_{4}^{\prime}}, \mathcal{Y}_{a_{1} a_{5} ; i}^{a_{4} ;(3)}\left(w_{a_{1}}, z_{1}\right) \mathcal{Y}_{a_{2} a_{3} ; j}^{a_{5} ;(4)}\left(w_{a_{2}}, z_{2}\right) w_{a_{3}}\right\rangle
\end{gathered}
$$

when $\left|z_{1}\right|>\left|z_{2}\right|>\left|z_{1}-z_{2}\right|>0$, for $a_{1}, \ldots, a_{4}, a_{6} \in \mathcal{A}, w_{a_{1}} \in W^{a_{1}}, w_{a_{2}} \in W^{a_{2}}$, $w_{a_{3}} \in W^{a_{3}}, w_{a_{4}^{\prime}} \in W^{a_{4}^{\prime}}=\left(W^{a_{4}}\right)^{\prime}, k=1, \ldots, N_{a_{1} a_{2}}^{a_{6}}$ and $l=1, \ldots, N_{a_{6} a_{3}}^{a_{4}}$. These numbers are matrix elements of the inverse of the fusing isomorphism.

By Lemma 4.1 in [H5] or by the differential equations given by Theorem 1.4 in [H6], we know that

$$
\begin{gathered}
\left\langle w_{a_{4}^{\prime}}, \mathcal{Y}_{a_{1} a_{5} ; i}^{a_{4} ;(1)}\left(w_{a_{1}}, z_{1}\right) \mathcal{Y}_{a_{2} a_{3} ; j}^{a_{5} ;(2)}\left(w_{a_{2}}, z_{2}\right) w_{a_{3}}\right\rangle, \\
\left.\left\langle w_{a_{4}^{\prime}}, \mathcal{Y}_{a_{6} a_{3} ; l}^{a_{4} ;(3)}\left(\mathcal{Y}_{a_{1} a_{2} ; k}^{a_{6} ;(4)}\left(w_{a_{1}}, z_{1}-z_{2}\right) w_{a_{2}}, z_{2}\right) w_{a_{3}}\right\rangle\right\rangle, \\
\left\langle w_{a_{4}^{\prime}}, \mathcal{Y}_{a_{2} a_{6} ; k}^{a_{4} ;(5)}\left(w_{a_{2}}, z_{2}\right) \mathcal{Y}_{a_{1} a_{3} ; l}^{a_{6} ;(6)}\left(w_{a_{1}}, z_{1}\right) w_{a_{3}}\right\rangle
\end{gathered}
$$

can all be analytically extended to multi-valued analytic functions on

$$
M^{2}=\left\{\left(z_{1}, z_{2}\right) \in \mathbb{C}^{2} \mid z_{1}, z_{2} \neq 0, z_{1} \neq z_{2}\right\} .
$$

We can lift multi-valued analytic functions on $M^{2}$ to single-valued analytic functions on the universal covering $\widetilde{M^{2}}$ of $M^{2}$. However, these single-valued liftings are not unique. To obtain a unique lifting, we have to give singlevalued branches of the multi-valued analytic functions in simply-connected subsets of $M^{2}$.

Consider the following three regions:

$$
\begin{array}{r}
M^{2}\left(\left|z_{1}\right|>\left|z_{2}\right|>0,0 \leq \arg z_{1}, \arg z_{2}<2 \pi\right) \\
M^{2}\left(\left|z_{2}\right|>\left|z_{1}\right|>0,0 \leq \arg z_{1}, \arg z_{2}<2 \pi\right) \\
M^{2}\left(\left|z_{2}\right|>\left|z_{1}-z_{2}\right|>0,0 \leq \arg z_{2}, \arg \left(z_{1}-z_{2}\right)<2 \pi\right) .
\end{array}
$$


These are, respectively, the region $\left|z_{1}\right|>\left|z_{2}\right|>0$ with cuts along the real lines in the $z_{1}$ - and $z_{2}$-planes; the region $\left|z_{2}\right|>\left|z_{1}\right|>0$ with cuts along the real lines in the $z_{1}$ - and $z_{2}$-planes; the region $\left|z_{2}\right|>\left|z_{1}-z_{2}\right|>0$ with cuts along the real lines in the $z_{2}$ - and $z_{1}-z_{2}$-planes. The multi-valued analytic extensions of (1.7) and (1.8) have single-valued branches (1.7) and (1.8), respectively, on (1.10) and (1.12), respectively. Thus we have the corresponding single-valued analytic functions on $\widetilde{M}^{2}$. We denote these analytic functions by

$$
E\left(\left\langle w_{a_{4}^{\prime}}, \mathcal{Y}_{a_{1} a_{5} ; i}^{a_{4} ;(1)}\left(w_{a_{1}}, x_{1}\right) \mathcal{Y}_{a_{2} a_{3} ; j}^{a_{5} ;(2)}\left(w_{a_{2}}, x_{2}\right) w_{a_{3}}\right\rangle\right)
$$

and

$$
E\left(\left\langle w_{a_{4}^{\prime}}, \mathcal{Y}_{a_{6} a_{3} ; l}^{a_{4} ;(3)}\left(\mathcal{Y}_{a_{1} a_{2} ; k}^{a_{6} ;(4)}\left(w_{a_{1}}, z_{1}-z_{2}\right) w_{a_{2}}, z_{2}\right) w_{a_{3}}\right\rangle\right)
$$

respectively.

Then from (1.5) and (1.6), we immediately obtain

$$
\begin{aligned}
& E\left(\left\langle w_{a_{4}^{\prime}}, \mathcal{Y}_{a_{1} a_{5} ; i}^{a_{4} ;(1)}\left(w_{a_{1}}, z_{1}\right) \mathcal{Y}_{a_{2} a_{3} ; j}^{a_{5} ;(2)}\left(w_{a_{2}}, z_{2}\right) w_{a_{3}}\right\rangle\right) \\
&=\sum_{a_{6} \in \mathcal{A}} \sum_{k=1}^{N_{a_{1} a_{2}}^{a_{2}}} \sum_{l=1}^{N_{a_{6} a_{3}}^{a_{4}}} F\left(\mathcal{Y}_{a_{1} a_{5} ; i}^{a_{4} ;(1)} \otimes \mathcal{Y}_{a_{2} a_{3} ; j}^{a_{5} ;(2)} ; \mathcal{Y}_{a_{6} a_{3} ; l}^{a_{4} ;(3)} \otimes \mathcal{Y}_{a_{1} a_{2} ; k}^{a_{6} ;(4)}\right) \\
& \cdot E\left(\left\langle w_{a_{4}^{\prime}}, \mathcal{Y}_{a_{6} a_{3} ; l}^{a_{4} ;(3)}\left(\mathcal{Y}_{a_{1} a_{2} ; k}^{a_{6} ;(4)}\left(w_{a_{1}}, z_{1}-z_{2}\right) w_{a_{2}}, z_{2}\right) w_{a_{3}}\right\rangle\right)
\end{aligned}
$$

and

$$
\begin{gathered}
E\left(\left\langle w_{a_{4}^{\prime}}, \mathcal{Y}_{a_{6} a_{3} ; l}^{a_{4} ;(1)}\left(\mathcal{Y}_{a_{1} a_{2} ; k}^{a_{6} ;(2)}\left(w_{a_{1}}, z_{1}-z_{2}\right) w_{a_{2}}, z_{2}\right) w_{a_{3}}\right\rangle\right) \\
=\sum_{a_{5} \in \mathcal{A}} \sum_{i=1}^{N_{a_{1} a_{5}}^{a_{4} a_{5}}} \sum_{j=1}^{N_{a_{2} a_{3}}^{a_{2}}} F^{-1}\left(\mathcal{Y}_{a_{6} a_{3} ; l}^{a_{4} ;(1)} \otimes \mathcal{Y}_{a_{1} a_{2} ; k}^{a_{6} ;(2)} ; \mathcal{Y}_{a_{1} a_{5} ; i}^{a_{4} ;(3)} \otimes \mathcal{Y}_{a_{2} a_{3} ; j}^{a_{5} ;(4)}\right) \\
\cdot E\left(\left\langle w_{a_{4}^{\prime}}, \mathcal{Y}_{a_{1} a_{5} ; i}^{a_{4} ;(3)}\left(w_{a_{1}}, z_{1}\right) \mathcal{Y}_{a_{2} a_{3} ; j}^{a_{5} ;(4)}\left(w_{a_{2}}, z_{2}\right) w_{a_{3}}\right\rangle\right) .
\end{gathered}
$$

From a single-valued analytic function $f$ on $\widetilde{M^{2}}$, we can construct other single-valued analytic functions on $\widetilde{M}^{2}$ such that they and $f$ correspond to the same multi-valued analytic functions on $M^{2}$. One particularly interesting construction is given by a braiding operation. To give this construction, we need only give a single-valued branch of the multi-valued analytic function on $M^{2}$ corresponding to $f$ on a simply-connected region in $M^{2}$. We can view the region (1.11) as a simply-connected region in $\widetilde{M}^{2}$. Thus $f$ gives a singlevalued function on this region. For any $r \in \mathbb{Z}$, we now give a single-valued 
analytic functions on the region (1.10) in the following way: Consider the path

$$
t \mapsto\left(\frac{3}{2}-\frac{e^{(2 r+1) \pi i t}}{2}, \frac{3}{2}+\frac{e^{(2 r+1) \pi i t}}{2}\right)
$$

from the point $(1,2)$ in the region (1.11) to the point $(2,1)$ in the region (1.10). This path and the restriction of $f$ to the region (1.11) gives a unique single-valued analytic function on the region (1.10). This single-valued analytic function on this simply-connected region determines uniquely a singlevalued analytic function $B^{(r)}(f)$ on $\widetilde{M}^{2}$ such that $f$ and $B^{(r)}(f)$ correspond to the same multi-valued analytic function on $M^{2}$.

We now consider (1.9). From the discussion above, we have a singlevalued analytic function

$$
E\left(\left\langle w_{a_{4}^{\prime}}, \mathcal{Y}_{a_{2} a_{6} ; k}^{a_{4} ;(5)}\left(w_{a_{2}}, z_{2}\right) \mathcal{Y}_{a_{1} a_{3} ; l}^{a_{6} ;(6)}\left(w_{a_{1}}, z_{1}\right) w_{a_{3}}\right\rangle\right)
$$

on $\widetilde{M^{2}}$. Apply our construction above, we obtain another single-valued analytic function

$$
B^{(r)}\left(E\left(\left\langle w_{a_{4}^{\prime}}, \mathcal{Y}_{a_{2} a_{6} ; k}^{a_{4} ;(3)}\left(w_{a_{2}}, z_{2}\right) \mathcal{Y}_{a_{1} a_{3} ; l}^{a_{6} ;(4)}\left(w_{a_{1}}, z_{1}\right) w_{a_{3}}\right\rangle\right)\right)
$$

on $\widetilde{M}^{2}$. In terms of these functions, the commutativity for intertwining operators (see Theorem 3.1 in [H2] and Proposition 2.2 [H5]) can be written as

$$
\begin{gathered}
B^{(r)}\left(E\left(\left\langle w_{a_{4}^{\prime}}, \mathcal{Y}_{a_{1} a_{5} ; i}^{a_{4} ;(1)}\left(w_{a_{1}}, z_{2}\right) \mathcal{Y}_{a_{2} a_{3} ; j}^{a_{5} ;(2)}\left(w_{a_{2}}, z_{1}\right) w_{a_{3}}\right\rangle\right)\right) \\
=\sum_{a_{6} \in \mathcal{A}} \sum_{k=1}^{N_{a_{2} a_{6}}^{a_{4}}} \sum_{l=1}^{N_{a_{1} a_{3}}^{a_{3}}} B^{(r)}\left(\mathcal{Y}_{a_{1} a_{5} ; i}^{a_{4} ;(1)} \otimes \mathcal{Y}_{a_{2} a_{3} ; j}^{a_{5} ;(2)} ; \mathcal{Y}_{a_{2} a_{6} ; k}^{a_{4} ;(3)} \otimes \mathcal{Y}_{a_{1} a_{3} ; l}^{a_{6} ;(4)}\right) \\
\cdot E\left(\left\langle w_{a_{4}^{\prime}}, \mathcal{Y}_{a_{2} a_{6} ; k}^{a_{4} ;(3)}\left(w_{a_{2}}, z_{1}\right) \mathcal{Y}_{a_{1} a_{3} ; l}^{a_{6} ;(4)}\left(w_{a_{1}}, z_{2}\right) w_{a_{3}}\right\rangle\right),
\end{gathered}
$$

where the numbers

$$
B^{(r)}\left(\mathcal{Y}_{a_{1} a_{5} ; i}^{a_{4} ;(1)} \otimes \mathcal{Y}_{a_{2} a_{3} ; j}^{a_{5} ;(2)} ; \mathcal{Y}_{a_{2} a_{6} ; k}^{a_{4} ;(3)} \otimes \mathcal{Y}_{a_{1} a_{3} ; l}^{a_{6} ;(4)}\right)
$$

are the matrix elements of the braiding isomorphism.

From the construction above, it is easy to see that the square $\left(B^{(r)}\right)^{2}$ of $B^{(r)}$ is actually the map sending a single-valued analytic function $f$ on $\widetilde{M}^{2}$ 
to another one $\left(B^{(r)}\right)^{2}(f)$ corresponding to the same multi-valued analytic functions on $M^{2}$ in the following way: Consider the path

$$
t \mapsto\left(\frac{3}{2}+\frac{e^{2(2 r+1) \pi i t}}{2}, \frac{3}{2}-\frac{e^{2(2 r+1) \pi i t}}{2}\right)
$$

from the point $(2,1)$ to itself. This path and the restriction of $f$ to the region (1.10) gives another single-valued analytic function on the same region. This new single-valued analytic function on the region (1.10) gives a single-valued analytic function $\left(B^{(r)}\right)^{2}(f)$ on $\widetilde{M}^{2}$. From this description of $\left(B^{(r)}\right)^{2}(f)$, we see that $\left(B^{(r)}\right)^{2}(f)$ is in fact the monodromy of the multi-valued function corresponding to $f$ given by $\log \left(z_{1}-z_{2}\right) \mapsto \log \left(z_{1}-z_{2}\right)+2(2 r+1) \pi i$.

We shall also use similar notations to denote the matrix elements of the square $\left(B^{(r)}\right)^{2}$ of $B^{(r)}$ under the bases above as

$$
\left(B^{(r)}\right)^{2}\left(\mathcal{Y}_{a_{1} a_{5} ; i}^{a_{4} ;(1)} \otimes \mathcal{Y}_{a_{2} a_{3} ; j}^{\left.a_{5} ; 2\right)} ; \mathcal{Y}_{a_{1} a_{6} ; i}^{a_{4} ;(3)} \otimes \mathcal{Y}_{a_{2} a_{3} ; j}^{a_{6} ;(4)}\right)
$$

that is,

$$
\begin{gathered}
\left(B^{(r)}\right)^{2}\left(E\left(\left\langle w_{a_{4}^{\prime}}, \mathcal{Y}_{a_{1} a_{5} ; i}^{a_{4} ;(1)}\left(w_{a_{1}}, z_{1}\right) \mathcal{Y}_{a_{2} a_{3} ; j}^{a_{5} ;(2)}\left(w_{a_{2}}, z_{2}\right) w_{a_{3}}\right\rangle\right)\right) \\
=\sum_{a_{6} \in \mathcal{A}} \sum_{k=1}^{N_{a_{2} a_{6}}^{a_{4}}} \sum_{l=1}^{N_{a_{1} a_{3}}^{a_{6}}}\left(B^{(r)}\right)^{2}\left(\mathcal{Y}_{a_{1} a_{5} ; i}^{a_{4} ;(1)} \otimes \mathcal{Y}_{a_{2} a_{3} ; j}^{a_{5} ;(2)} ; \mathcal{Y}_{a_{1} a_{6} ; i}^{a_{4} ;(3)} \otimes \mathcal{Y}_{a_{2} a_{3} ; j}^{a_{6} ;(4)}\right) \\
\cdot E\left(\left\langle w_{a_{4}^{\prime}}, \mathcal{Y}_{a_{1} a_{6} ; i}^{a_{4} ;(1)}\left(w_{a_{1}}, z_{1}\right) \mathcal{Y}_{a_{2} a_{3} ; j}^{a_{6} ;(2)}\left(w_{a_{2}}, z_{2}\right) w_{a_{3}}\right\rangle\right) .
\end{gathered}
$$

As in the case of correlation functions constructed from products and iterates of intertwining operators above, if we have a series $\varphi$ which is absolutely convergent in a region in $M^{2}$ and can be analytically extended to a multi-valued analytic function on $M^{2}$, then we obtain a unique single-valued analytic function $E(\varphi)$ on $\widetilde{M}^{2}$. For example, we know that for $z \in \mathbb{C}$,

$$
\left\langle w_{a_{4}^{\prime}}, \mathcal{Y}_{a_{1} a_{5} ; i}^{a_{4} ;(1)}\left(w_{a_{1}}, z_{1}\right) e^{z L(-1)} \mathcal{Y}_{a_{2} a_{3} ; j}^{a_{5} ;(2)}\left(w_{a_{2}}, z_{2}-z\right) e^{-z L(-1)} w_{a_{3}}\right\rangle
$$

is absolutely convergent in the region given by $\left|z_{1}\right|>\left|z_{2}\right|>0$ and $\left|z_{2}-z\right|>|z|$ and it can be analytically extended to a multi-valued analytic function on $M^{2}$. Thus we have a single-valued analytic function

$$
E\left(\left\langle w_{a_{4}^{\prime}}, \mathcal{Y}_{a_{1} a_{5} ; i}^{a_{4} ;(1)}\left(w_{a_{1}}, z_{1}\right) e^{z L(-1)} \mathcal{Y}_{a_{2} a_{3} ; j}^{a_{5} ;(2)}\left(w_{a_{2}}, z_{2}-z\right) e^{-z L(-1)} w_{a_{3}}\right\rangle\right)
$$


on $\widetilde{M}^{2}$. All the other properties of intertwining operators, for example, the skew-symmetry, the contragredient intertwining operators and so on, can all be expressed using equalities for such single-valued analytic functions on $\widetilde{M^{2}}$.

We shall need the following result:

Proposition 1.2 For $a_{1}, a_{2}, a_{3}, a_{4} \in \mathcal{A}$, the maps from $W^{a_{4}^{\prime}} \otimes W^{a_{1}} \otimes W^{a_{2}} \otimes$ $W^{a_{3}}$ to the space of single-valued analytic functions on $\widetilde{M}^{2}$ given by

$$
w_{a_{4}^{\prime}} \otimes w_{a_{1}} \otimes w_{a_{2}} \otimes w_{a_{3}} \mapsto E\left(\left\langle w_{a_{4}^{\prime}}, \mathcal{Y}_{a_{1} a_{5} ; i}^{a_{4} ;(1)}\left(w_{a_{1}}, z_{1}\right) \mathcal{Y}_{a_{2} a_{3} ; j}^{a_{5} ;(2)}\left(w_{a_{2}}, z_{2}\right) w_{a_{3}}\right\rangle\right),
$$

$a_{5} \in \mathcal{A}, i=1, \ldots, N_{a_{1} a_{5}}^{a_{4}}, j=1, \ldots, N_{a_{2} a_{3}}^{a_{5}}$, are linearly independent. Similarly, for $a_{1}, a_{2}, a_{3}, a_{4} \in \mathcal{A}$, the maps from $W^{a_{4}^{\prime}} \otimes W^{a_{1}} \otimes W^{a_{2}} \otimes W^{a_{3}}$ to the space of single-valued analytic functions on $\widetilde{M}^{2}$ given by

$$
w_{a_{4}^{\prime}} \otimes w_{a_{1}} \otimes w_{a_{2}} \otimes w_{a_{3}} \mapsto E\left(\left\langle w_{a_{4}^{\prime}}, \mathcal{Y}_{a_{6} a_{3} ; k}^{a_{4} ;(3)}\left(\mathcal{Y}_{a_{1} a_{2} ; l}^{\left.a_{6} ; 4\right)}\left(w_{a_{1}}, z_{1}-z_{2}\right) w_{a_{2}}, z_{2}\right) w_{a_{3}}\right\rangle\right),
$$

$a_{6} \in \mathcal{A}, k=1, \ldots, N_{a_{6} a_{3}}^{a_{4}}, l=1, \ldots, N_{a_{1} a_{2}}^{a_{6}}$, are linearly independent.

Proof. We prove only the linear independence of the maps obtained from products of intertwining operators. For iterates, the proof is similar.

Since analytic extensions are unique, we need only prove that the maps from $W^{a_{4}^{\prime}} \otimes W^{a_{1}} \otimes W^{a_{2}} \otimes W^{a_{3}}$ to the space of the single-valued analytic functions on the region (1.10) given by

$$
w_{a_{4}^{\prime}} \otimes w_{a_{1}} \otimes w_{a_{2}} \otimes w_{a_{3}} \mapsto\left\langle w_{a_{4}^{\prime}}, \mathcal{Y}_{a_{1} a_{5} ; i}^{a_{4} ;(1)}\left(w_{a_{1}}, z_{1}\right) \mathcal{Y}_{a_{2} a_{3} ; j}^{a_{5} ;(2)}\left(w_{a_{2}}, z_{2}\right) w_{a_{3}},\right\rangle,
$$

$a_{5} \in \mathcal{A}, i=1, \ldots, N_{a_{1} a_{5}}^{a_{4}}, j=1, \ldots, N_{a_{2} a_{3}}^{a_{5}}$, are linearly independent. Assume that

$$
\sum_{a_{5} \in \mathcal{A}} \sum_{i=1}^{N_{a_{1} a_{5}}^{a_{4} a_{5}}} \sum_{j=1}^{N_{a_{2} a_{3}}^{a_{5}}} \lambda_{a_{5}, i, j}\left\langle w_{a_{4}^{\prime}}, \mathcal{Y}_{a_{1} a_{5} ; i}^{a_{4} ;(1)}\left(w_{a_{1}}, z_{1}\right) \mathcal{Y}_{a_{2} a_{3} ; j}^{a_{5} ;(2)}\left(w_{a_{2}}, z_{2}\right) w_{a_{3}}\right\rangle=0
$$

Since (1.16) holds for all $z_{1}$ and $z_{2}$ satisfying $\left|z_{1}\right|>\left|z_{2}\right|>0$, we obtain the following equation in formal variables:

$$
\sum_{a_{5} \in \mathcal{A}} \sum_{i=1}^{N_{a_{1} a_{5}}^{a_{4}}} \sum_{j=1}^{N_{a_{2} a_{3}}^{a_{5} a_{3}}} \lambda_{a_{5}, i, j}\left\langle w_{a_{4}^{\prime}}, \mathcal{Y}_{a_{1} a_{5} ; i}^{a_{4} ;(1)}\left(w_{a_{1}}, x_{1}\right) \mathcal{Y}_{a_{2} a_{3} ; j}^{a_{5} ;(2)}\left(w_{a_{2}}, x_{2}\right) w_{a_{3}}\right\rangle=0
$$

We want to show that $\lambda_{a_{5}, i, j}=0$ for $a_{5} \in \mathcal{A}, i=1, \ldots, N_{a_{1} a_{5}}^{a_{4}}$ and $j=$ $1, \ldots, N_{a_{2} a_{3}}^{a_{5}}$. 
From the tensor product theory in [HL1] and [HL4], we know that the tensor product module $W^{a_{2}} \bigotimes_{P\left(z_{2}\right)} W^{a_{3}}$ is isomorphic to $\oplus_{a_{5} \in \mathcal{A}} N_{a_{2} a_{3}}^{a_{5}} W^{a_{5}}$. For $a_{5} \in \mathcal{A}$ and $j=1, \ldots, N_{a_{2} a_{3}}^{a_{5}}$, let $\pi_{a_{5} ; j}$ be the projections from $\oplus_{a_{5} \in \mathcal{A}} N_{a_{2} a_{3}}^{a_{5}} W^{a_{5}}$ to the $j$-th copy of $W^{a_{5}}$. Let $f: W^{a_{2}} \bigotimes_{P\left(z_{2}\right)} W^{a_{3}} \rightarrow \oplus_{a_{5} \in \mathcal{A}} N_{a_{2} a_{3}}^{a_{5}} W^{a_{5}}$ be the isomorphism such that

$$
\bar{\pi}_{a_{5} ; j}\left(\bar{f}\left(w_{a_{2}} \bigotimes_{P\left(z_{2}\right)} w_{a_{3}}\right)\right)=\mathcal{Y}_{a_{2} a_{3} ; j}^{a_{5} ;(2)}\left(w_{a_{2}}, z_{2}\right) w_{a_{3}}
$$

for $w_{a_{2}} \in W^{a_{2}}$ and $w_{a_{3}} \in W^{a_{3}}$, where

$$
\bar{\pi}_{a_{5} ; j}: \overline{\oplus_{a_{5} \in \mathcal{A}} N_{a_{2} a_{3}}^{a_{5}} W^{a_{5}}} \rightarrow \bar{W}^{a_{5}}
$$

and

$$
\bar{f}: \overline{W^{a_{2}} \bigotimes_{P\left(z_{2}\right)} W^{a_{3}}} \rightarrow \overline{\oplus_{a_{5} \in \mathcal{A}} N_{a_{2} a_{3}}^{a_{5}} W^{a_{5}}}
$$

are the natural extensions of $\pi_{a_{5} ; j}$ and $f$. By the universal property for the tensor product module, such $f$ indeed exists. Let $\mathcal{Y}_{2}$ be the intertwining operator corresponding to the intertwining map $\otimes_{P\left(z_{2}\right)}: W^{a_{2}} \otimes W^{a_{3}} \rightarrow$ $W^{a_{2}} \bigotimes_{P\left(z_{2}\right)} W^{a_{3}}$ (see [HL1] and [HL4]). Then we have

$$
\pi_{a_{5} ; j}\left(f\left(\mathcal{Y}_{2}\left(w_{a_{2}}, x\right) w_{a_{3}}\right)\right)=\mathcal{Y}_{a_{2} a_{3} ; j}^{a_{5} ;(2)}\left(w_{a_{2}}, x\right) w_{a_{3}}
$$

for $w_{a_{2}} \in W_{W^{a_{4}}}^{a_{2}}$ and $w_{a_{3}} \in W^{a_{3}}$. Let $\mathcal{Y}_{1}$ be the intertwining operator of type

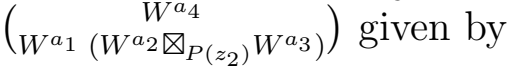

$$
\mathcal{Y}_{1}\left(w_{a_{1}}, x\right) w=\sum_{a_{5} \in \mathcal{A}} \sum_{i=1}^{N_{a_{1} a_{5}}^{a_{4}}} \sum_{j=1}^{N_{a_{2} a_{3}}^{a_{5}}} \lambda_{a_{5}, i, j} \mathcal{Y}_{a_{1} a_{5} ; i}^{a_{4} ;(1)}\left(w_{a_{1}}, x\right) \pi_{a_{5} ; j}(f(w))
$$

for $w_{a_{1}} \in W^{a_{1}}$ and $w \in W^{a_{2}} \otimes_{P\left(z_{2}\right)} W^{a_{3}}$.

By (1.18) and (1.19), the left-hand side of (1.17) is equal to

$$
\begin{gathered}
\sum_{a_{5} \in \mathcal{A}} \sum_{i=1}^{N_{a_{1} a_{5}}^{a_{4}}} \sum_{j=1}^{N_{a_{2} a_{3}}^{a_{5}}} \lambda_{a_{5}, i, j}\left\langle w_{a_{4}^{\prime}}, \mathcal{Y}_{a_{1} a_{5} ; i}^{a_{4} ;(1)}\left(w_{a_{1}}, x_{1}\right) \pi_{a_{5} ; j}\left(f\left(\mathcal{Y}_{2}\left(w_{a_{2}}, x_{2}\right) w_{a_{3}}\right)\right)\right\rangle \\
=\left\langle w_{a_{4}^{\prime}}, \mathcal{Y}_{1}\left(w_{a_{1}}, x_{1}\right) \mathcal{Y}_{2}\left(w_{a_{2}}, x_{2}\right) w_{a_{3}}\right\rangle .
\end{gathered}
$$

Thus we have

$$
\left\langle w_{a_{4}^{\prime}}, \mathcal{Y}_{1}\left(w_{a_{1}}, x_{1}\right) \mathcal{Y}_{2}\left(w_{a_{2}}, x_{2}\right) w_{a_{3}}\right\rangle=0
$$

for $w_{a_{4}^{\prime}} \in W^{a_{4}^{\prime}}, w_{a_{1}} \in W^{a_{1}}, w_{a_{2}} \in W^{a_{2}}, w_{a_{3}} \in W^{a_{3}}$. Since the homogeneous components of $w_{a_{2}} \otimes_{P\left(z_{2}\right)} w_{a_{3}}$ or equivalently the $x_{2}$-coefficients of 
$\mathcal{Y}_{2}\left(w_{a_{2}}, x_{2}\right) w_{a_{3}}$ for $w_{a_{2}} \in W^{a_{2}}$ and $w_{a_{3}} \in W^{a_{3}} \operatorname{span} W^{a_{2}} \bigotimes_{P\left(z_{2}\right)} W^{a_{3}}$ (see [H1]), we obtain from (1.20) that

$$
\left\langle w_{a_{4}^{\prime}}, \mathcal{Y}\left(w_{a_{1}}, x_{1}\right) w\right\rangle=0 .
$$

for $w_{a_{4}^{\prime}} \in W^{a_{4}^{\prime}}, w_{a_{1}} \in W^{a_{1}}$ and $w \in W^{a_{2}} \nabla_{P\left(z_{2}\right)} W^{a_{3}}$.

Now take $w$ to be an element of $W^{a_{2}} \bigotimes_{P\left(z_{2}\right)} W^{a_{3}}$ such that $f(w)$ is in the $j$-th copy of $W^{a_{5}}$ in $\oplus_{a_{5} \in \mathcal{A}} N_{a_{2} a_{3}}^{a_{5}} W^{a_{5}}$, that is, take $w$ such that $\pi_{a_{5} ; j}(f(w))=$ $f(w), \pi_{a_{5} ; m}(f(w))=0$ for $m \neq j$ and $\pi_{a ; m}(f(w))=0$ for $a \neq a_{5}$. Then we have

$$
\sum_{i=1}^{N_{a_{1} a_{5}}^{a_{4}}} \lambda_{a_{5}, i, j}\left\langle w_{a_{4}^{\prime}}, \mathcal{Y}_{a_{1} a_{5} ; i}^{a_{4} ;(1)}\left(w_{a_{1}}, x_{1}\right) f(w)\right\rangle=0 .
$$

Since $w_{a_{4}^{\prime}}, w_{a_{1}}, w$ are arbitrary elements of $W^{a_{4}^{\prime}}, W^{a_{1}}$ and the $j$-th copy of $W^{a_{5}}$ in $\oplus_{a_{5} \in \mathcal{A}} N_{a_{2} a_{3}}^{a_{5}} W^{a_{5}}$, respectively, we obtain

$$
\sum_{i=1}^{N_{a_{4} a_{5}}^{a_{4}}} \lambda_{a_{5}, i, j} \mathcal{Y}_{a_{1} a_{5} ; i}^{a_{4} ;(1)}=0
$$

Since $\mathcal{Y}_{a_{1} a_{5} ; i}^{a_{4} ;(1)}$ for $i=1, \ldots, N_{a_{1} a_{5}}^{a_{4}}$ are linearly independent, we obtain $\lambda_{a_{5}, i, j}=$ 0 for $a_{5} \in \mathcal{A}, i=1, \ldots, N_{a_{1} a_{5}}^{a_{4}}$ and $j=1, \ldots, N_{a_{2} a_{3}}^{a_{5}}$.

Since

$$
\left\langle w_{a_{2}^{\prime}}, \mathcal{Y}_{a_{2} ; 1}^{a_{2}}\left(w_{a_{2}}^{1}, z_{1}\right) \mathcal{Y}_{a_{3}^{\prime} a_{3} ; 1}^{e}\left(w_{a_{3}^{\prime}}, z_{2}\right) \mathcal{Y}_{a_{1} a_{2} ; i}^{a_{3}}\left(w_{a_{1}}, z_{3}\right) w_{a_{2}}^{2}\right\rangle
$$

and

$$
\left\langle w_{a_{2}^{\prime}}, \mathcal{Y}_{a_{4} a_{3} ; k}^{a_{2}}\left(\mathcal{Y}_{a_{2} a_{3}^{\prime} ; j}^{a_{4}}\left(w_{a_{2}}^{1}, z_{1}-z_{2}\right) w_{a_{3}^{\prime}}, z_{2}\right) \mathcal{Y}_{a_{1} a_{2} ; i}^{a_{3}}\left(w_{a_{1}}, z_{3}\right) w_{a_{2}}^{2}\right\rangle
$$

satisfy a system of differential equations of regular singular points with coefficients in

$$
\mathbb{C}\left[z_{1}, z_{2}^{-1}, z_{2}, z_{2}^{-1}, z_{3}, z_{3}^{-1},\left(z_{1}-z_{2}\right)^{-1},\left(z_{1}-z_{3}\right)^{-1},\left(z_{2}-z_{3}\right)^{-1}\right]
$$

(see [H6]), they are absolutely convergent in the region given by $\left|z_{1}\right|>\left|z_{2}\right|>$ $\left|z_{3}\right|>0$ and in the region given by $\left|z_{2}\right|>\left|z_{1}-z_{2}\right|>0,\left|z_{2}\right|>\left|z_{3}\right|>0$, $\left|z_{2}-z_{3}\right|>\left|z_{1}-z_{2}\right|>0$, respectively. Using also these differential equations, 
we see that (1.21) and (1.22) can also be analytically extended to multi-valued analytic functions on

$$
M^{3}=\left\{\left(z_{1}, z_{2}, z_{3}\right) \in \mathbb{C}^{3} \mid z_{1}, z_{2}, z_{3} \neq 0, z_{1} \neq z_{2}, z_{2} \neq z_{3}, z_{1} \neq z_{3}\right\} .
$$

We can also choose single valued branches of these multi-valued analytic functions on the region given by $\left|z_{1}\right|>\left|z_{2}\right|>\left|z_{3}\right|>0$ with cuts along the real lines in the $z_{1^{-}}, z_{2^{-}}$and $z_{3}$-planes and on the region $\left|z_{2}\right|>\left|z_{1}-z_{2}\right|>0$, $\left|z_{2}\right|>\left|z_{3}\right|>0,\left|z_{2}-z_{3}\right|>\left|z_{1}-z_{2}\right|>0$ with cuts along the real lines in the $z_{2^{-}}, z_{1}-z_{2^{-}}$and $z_{3^{-}}$-planes.

Similarly to the case of two intertwining operators above, we also have the single-valued analytic functions

$$
E\left(\left\langle w_{a_{2}^{\prime}}, \mathcal{Y}_{a_{2} e ; 1}^{a_{2}}\left(w_{a_{2}}^{1}, z_{1}\right) \mathcal{Y}_{a_{3}^{\prime} a_{3} ; 1}^{e}\left(w_{a_{3}^{\prime}}, z_{2}\right) \mathcal{Y}_{a_{1} a_{2} ; i}^{a_{3}}\left(w_{a_{1}}, z_{3}\right) w_{a_{2}}^{2}\right\rangle\right)
$$

and

$$
E\left(\left\langle w_{a_{2}^{\prime}}, \mathcal{Y}_{a_{4} a_{3} ; k}^{a_{2}}\left(\mathcal{Y}_{a_{2} a_{3}^{\prime} ; j}^{a_{4}}\left(w_{a_{2}}^{1}, z_{1}-z_{2}\right) w_{a_{3}^{\prime}}, z_{2}\right) \mathcal{Y}_{a_{1} a_{2} ; i}^{a_{3}}\left(w_{a_{1}}, z_{3}\right) w_{a_{2}}^{2}\right\rangle\right)
$$

on the universal covering $\widetilde{M}^{3}$ of $M^{3}$. The associativity can also be written using these single valued analytic functions. Similarly we have other single valued analytic functions on $\bar{M}^{3}$, for example,

$$
\begin{aligned}
& E\left(\left\langle w_{a_{2}^{\prime}}, \mathcal{Y}_{a_{4} a_{3} ; k}^{a_{2}}\left(\mathcal{Y}_{a_{2} a_{3}^{\prime} ; j}^{a_{4}}\left(w_{a_{2}}^{1}, z_{1}-z_{2}\right) w_{a_{3}^{\prime}}, z_{2}\right) \mathcal{Y}_{a_{1} a_{2} ; i}^{a_{3}}\left(w_{a_{1}}, z_{3}\right) w_{a_{2}}^{2}\right\rangle\right), \\
& B_{12}^{(r)}\left(E\left(\left\langle w_{a_{2}^{\prime}}, \mathcal{Y}_{a_{2} e ; 1}^{a_{2}}\left(w_{a_{2}}^{1}, z_{1}\right) \mathcal{Y}_{a_{3}^{\prime} a_{3} ; 1}^{e}\left(w_{a_{3}^{\prime}}, z_{2}\right) \mathcal{Y}_{a_{1} a_{2} ; i}^{a_{3}}\left(w_{a_{1}}, z_{3}\right) w_{a_{2}}^{2}\right\rangle\right)\right), \\
& B_{23}^{(r)}\left(E\left(\left\langle w_{a_{2}^{\prime}}, \mathcal{Y}_{a_{2} e ; 1}^{a_{2}}\left(w_{a_{2}}^{1}, z_{1}\right) \mathcal{Y}_{a_{3}^{\prime} a_{3} ; 1}^{e}\left(w_{a_{3}^{\prime}}, z_{2}\right) \mathcal{Y}_{a_{1} a_{2} ; i}^{a_{3}}\left(w_{a_{1}}, z_{3}\right) w_{a_{2}}^{2}\right\rangle\right)\right)
\end{aligned}
$$

and so on, where the subscripts 12 and 23 in $B_{12}^{(r)}$ and $B_{23}^{(r)}$, respectively, mean that they corresponding to braiding isomorphisms which braid the first two and the last two intertwining operators, respectively.

Similarly to the case of two intertwining operators, we also have the following result:

Proposition 1.3 For $a_{1}, a_{2}, a_{3}, a_{4}, a_{5} \in \mathcal{A}$, the maps from $W^{a_{5}^{\prime}} \otimes W^{a_{1}} \otimes$ $W^{a_{2}} \otimes W^{a_{3}} \otimes W^{a_{4}}$ to the space of single-valued analytic functions on $\widetilde{M}^{3}$ given by

$$
\begin{aligned}
& w_{a_{5}^{\prime}} \otimes w_{a_{1}} \otimes w_{a_{2}} \otimes w_{a_{3}} \otimes w_{a_{4}} \mapsto \\
& E\left(\left\langle w_{a_{4}^{\prime}}, \mathcal{Y}_{a_{6} a_{4} ; k}^{a_{5} ;(1)}\left(\mathcal{Y}_{a_{7} a_{3} ; l}^{a_{6} ;(2)}\left(\mathcal{Y}_{a_{1} a_{2} ; m}^{a_{7} ;(3)}\left(w_{a_{1}}, z_{1}-z_{2}\right) w_{a_{2}}, z_{2}-z_{3}\right) w_{a_{3}}, z_{3}\right) w_{a_{4}}\right\rangle\right)
\end{aligned}
$$


for $a_{6}, a_{7} \in \mathcal{A}, k=1, \ldots, N_{a_{6} a_{4}}^{a_{5}}, l=1, \ldots, N_{a_{7} a_{3}}^{a_{6}}$ and $m=1, \ldots, N_{a_{1} a_{2}}^{a_{7}}$ are linearly independent. Similarly, $a_{1}, a_{2}, a_{3}, a_{4}, a_{5} \in \mathcal{A}$, the maps from $W^{a_{5}^{\prime}} \otimes W^{a_{1}} \otimes W^{a_{2}} \otimes W^{a_{3}} \otimes W^{a_{4}}$ to the space of single-valued analytic functions on $\widetilde{M}^{3}$ given by the lifting to the universal covering of analytic extensions of products or combinations of products and iterates of bases of intertwining operators are linearly independent.

The proof of this proposition is similar to the proof of Proposition 1.2 and is omitted.

\section{Geometrically-modified intertwining oper- ators and genus-one correlation functions}

We now discuss genus-one correlation functions. In the present paper, we need only one or two point functions. Genus-one correlation functions are defined using what we called geometrically-modified intertwining operators (see [H7]). We shall first discuss these operators and prove some important properties needed in this paper.

Let $A_{j}, j \in \mathbb{Z}_{+}$, be the complex numbers defined by

$$
\frac{1}{2 \pi i} \log (1+2 \pi i y)=\left(\exp \left(\sum_{j \in \mathbb{Z}_{+}} A_{j} y^{j+1} \frac{\partial}{\partial y}\right)\right) y \text {. }
$$

For any $V$-module $W$, let

$$
\mathcal{U}(x)=(2 \pi i x)^{L(0)} e^{-L^{+}(A)} \in(\text { End } W)\{x\}
$$

where $L_{+}(A)=\sum_{j \in \mathbb{Z}_{+}} A_{j} L(j)$ and $L(j)$ for $j \in \mathbb{Z}$ are the Virasoro operators on $W$. Given an intertwining operator $\mathcal{Y}$ of type $\left(\begin{array}{c}W^{a_{3}} \\ W^{a_{1}} W^{a_{2}}\end{array}\right)$, the map $W^{a_{1}} \otimes W^{a_{2}} \rightarrow W^{a_{3}}\{x\}$ defined by $w_{a_{1}} \otimes w_{a_{2}} \mapsto \mathcal{Y}\left(\mathcal{U}(x) w_{a_{1}}, x\right) w_{a_{2}}$ is the corresponding geometrically-modified intertwining operator. We need the following property of geometrically-modified intertwining operators:

Proposition 2.1 Let $\mathcal{Y}$ be an intertwining operator of type $\left(\begin{array}{c}W^{a_{3}} \\ W^{a_{1}} W^{a_{2}}\end{array}\right)$. Then for $w_{a_{1}} \in W^{a_{1}}, w_{a_{1}} \in W^{a_{1}}$ and $w_{a_{3}}^{\prime} \in W^{a_{3}^{\prime}}$, we have

$$
\begin{aligned}
\left\langle w_{a_{3}}^{\prime}, \sigma_{23}(\mathcal{Y})\left(\mathcal{U}(x) w_{a_{1}}, x\right) w_{a_{2}}\right\rangle & =e^{\pi i h_{a_{1}}}\left\langle\mathcal{Y}\left(\mathcal{U}\left(x^{-1}\right) e^{-\pi i L(0)} w_{a_{1}}, x^{-1}\right) w_{a_{3}}^{\prime}, w_{a_{2}}\right\rangle \\
& =e^{-\pi i h_{a_{1}}}\left\langle\mathcal{Y}\left(\mathcal{U}\left(x^{-1}\right) e^{\pi i L(0)} w_{a_{1}}, x^{-1}\right) w_{a_{3}}^{\prime}, w_{a_{2}}\right\rangle
\end{aligned}
$$


Proof. We first prove the first equality in (2.2). By the definition of $\sigma_{23}(\mathcal{Y})$, we have

$$
\begin{aligned}
& \left\langle w_{a_{3}}^{\prime}, \sigma_{23}(\mathcal{Y})\left(\mathcal{U}(x) w_{a_{1}}, x\right) w_{a_{2}}\right\rangle \\
& \quad=e^{\pi i h_{a_{1}}}\left\langle\mathcal{Y}\left(e^{x L(1)}\left(e^{-\pi i} x^{-2}\right)^{L(0)} \mathcal{U}(x) w_{a_{1}}, x^{-1}\right) w_{a_{3}}^{\prime}, w_{a_{2}}\right\rangle .
\end{aligned}
$$

We now calculate $e^{x L(1)}\left(e^{-\pi i} x^{-2}\right)^{L(0)} \mathcal{U}(x) w$. The equality

$$
\frac{1}{2 \pi i} \log \left(1-\frac{x}{y^{-1}+x}\right)=-\frac{1}{2 \pi i} \log (1+x y)
$$

gives

$$
\begin{aligned}
& e^{-x y^{2} \frac{\partial}{\partial y}}\left(e^{-\pi i} x^{-2}\right)-y \frac{\partial}{\partial y}(2 \pi i x)-y \frac{\partial}{\partial y} \sum_{e^{j \in \mathbb{Z}_{+}}} A_{j} y^{j+1} \frac{\partial}{\partial y} y \\
& =\left(2 \pi i x^{-1}\right)-y \frac{\partial}{\partial y} \sum_{e^{j \in \mathbb{Z}_{+}}} A_{j} y^{j+1} \frac{\partial}{\partial y} e^{\pi i y \frac{\partial}{\partial y}} y .
\end{aligned}
$$

Using the method developed and results obtained in Chapters 4 and 5 of [H3], we obtain

$$
e^{x L(1)}\left(e^{-\pi i} x^{-2}\right)^{L(0)}(2 \pi i x)^{L(0)} e^{-L_{+}(A)}=\left(2 \pi i x^{-1}\right)^{L(0)} e^{-L_{+}(A)} e^{-\pi i L(0)},
$$

that is,

$$
e^{x L(1)}\left(e^{-\pi i} x^{-2}\right)^{L(0)} \mathcal{U}(x)=\mathcal{U}\left(x^{-1}\right) e^{-\pi i L(0)},
$$

Thus the right-hand side of $(2.3)$ is equal to

$$
e^{\pi i h_{a_{1}}}\left\langle\mathcal{Y}\left(\mathcal{U}\left(x^{-1}\right) e^{-\pi i L(0)} w_{a_{1}}, x^{-1}\right) w_{a_{3}}^{\prime}, w_{a_{2}}\right\rangle,
$$

proving the first equality in (2.2).

The second equality in (2.2) follows immediately from the first equality and the equality

$$
\begin{aligned}
e^{-\pi L(0)} w_{a_{1}} & =e^{\pi L(0)} e^{-2 \pi L(0)} w_{a_{1}} \\
& =e^{-2 \pi h_{a_{1}}} e^{\pi L(0)} w_{a_{1}}
\end{aligned}
$$


As in the preceding section, For $a_{1}, a_{2}, a_{3} \in \mathcal{A}$ and $p=1,2,3,4,5,6$, let $\mathcal{Y}_{a_{1} a_{2} ; i}^{a_{3}(;)}, i=1, \ldots, N_{a_{1} a_{2}}^{a_{3}}$, be bases of $\mathcal{V}_{a_{1} a_{2}}^{a_{3}}$. Let $q_{\tau}=e^{2 \pi i \tau}$ for $\tau \in \mathbb{H}$. We consider $q_{\tau}$-traces of geometrically-modified intertwining operators of the following form:

$$
\operatorname{Tr}_{W^{a_{2}}} \mathcal{Y}_{a_{1} a_{2} ; i}^{a_{2} ;(1)}\left(\mathcal{U}\left(e^{2 \pi i z}\right) w_{a_{1}}, e^{2 \pi i z}\right) q_{\tau}^{L(0)-\frac{c}{24}}
$$

for $a_{1}, a_{2} \in \mathcal{A}, i=1, \ldots, N_{a_{1} a_{2}}^{a_{2}}$. In [M] and [H7], it was shown that these $q$-traces are independent of $z$, are absolutely convergent when $0<\left|q_{\tau}\right|<1$ and can be analytically extended to analytic functions of $\tau$ in the upper-half plane. We shall denote the analytic extension of (2.4) by

$$
E\left(\operatorname{Tr}_{W^{a_{2}}} \mathcal{Y}_{a_{1} a_{2} ; i}^{a_{2} ;(1)}\left(\mathcal{U}\left(e^{2 \pi i z}\right) w_{a_{1}}, e^{2 \pi i z}\right) q_{\tau}^{L(0)-\frac{c}{24}}\right) .
$$

These are genus-one one-point correlation functions. In [M] and [H7], the following modular invariance property is also proved: For

$$
\left(\begin{array}{ll}
a & b \\
c & d
\end{array}\right) \in S L(2, \mathbb{Z}),
$$

let $\tau^{\prime}=\frac{a \tau+b}{c \tau+d}$. Then for fixed $a_{1} \in \mathcal{A}$, there exist unique $A_{a_{2}}^{a_{3}} \in \mathbb{C}$ for $a_{2}, a_{3} \in \mathcal{A}$ such that

$$
\begin{gathered}
\operatorname{Tr}_{W^{a_{2}}} \mathcal{Y}_{a_{1} a_{2} ; i}^{a_{2} ;(1)}\left(\mathcal{U}\left(e^{2 \pi i \frac{z}{c \tau+d}}\right)\left(\frac{1}{c \tau+d}\right)^{L(0)} w_{a_{1}}, e^{2 \pi i \frac{z}{c \tau+d}}\right) q_{\tau^{\prime}}^{L(0)-\frac{c}{24}} \\
=\sum_{j=1}^{N_{a_{1} a_{3}}^{a_{3}}} \sum_{a_{3} \in \mathcal{A}} A_{a_{2} ; i}^{a_{3} ; j} \operatorname{Tr}_{W^{a_{3}}} \mathcal{Y}_{a_{1} a_{3} ; j}^{a_{3} ;(1)}\left(\mathcal{U}\left(e^{2 \pi i z}\right) w_{a_{1}}, e^{2 \pi i z}\right) q_{\tau}^{L(0)-\frac{c}{24}}
\end{gathered}
$$

for $w_{a_{1}} \in W^{a_{1}}$ and $i=1, \ldots, N_{a_{1} a_{2}}^{a_{2}}$. This modular invariance property can be written in terms of the analytic extensions as follows:

$$
\begin{gathered}
E\left(\operatorname{Tr}_{W^{a_{2}}} \mathcal{Y}_{a_{1} a_{2} ; i}^{a_{2} ;(1)}\left(\mathcal{U}\left(e^{\frac{2 \pi i z}{c \tau+d}}\right)\left(\frac{1}{c \tau+d}\right)^{L(0)} w_{a_{1}}, e^{\frac{2 \pi i z}{c \tau+d}}\right) q_{\tau^{\prime}}^{L(0)-\frac{c}{24}}\right) \\
=\sum_{j=1}^{N_{a_{1} a_{3}}^{a_{3}}} \sum_{a_{3} \in \mathcal{A}} A_{a_{2}}^{a_{3}} E\left(\operatorname{Tr}_{W^{a_{3}}} \mathcal{Y}_{a_{1} a_{3} ; i}^{a_{3} ;(1)}\left(\mathcal{U}\left(e^{2 \pi i z}\right) w_{a_{1}}, e^{2 \pi i z}\right) q_{\tau}^{L(0)-\frac{c}{24}}\right) .
\end{gathered}
$$

We consider the space $\mathcal{F}_{1 ; 1}^{e}$ spanned by linear maps of the form

$$
\begin{aligned}
\Psi_{a}: V & \rightarrow \mathbb{G}_{1 ; 1}^{e} \\
u & \mapsto \Psi_{a}(u ; \tau)
\end{aligned}
$$


for $a \in \mathcal{A}$, where

$$
\Psi_{a}(u ; \tau)=E\left(\operatorname{Tr}_{W^{a}} \mathcal{Y}_{e a ; 1}^{a ;(1)}\left(\mathcal{U}\left(e^{2 \pi i z}\right) u, e^{2 \pi i z}\right) q_{\tau}^{L(0)-\frac{c}{24}}\right)
$$

and $\mathbb{G}_{1 ; 1}^{e}$ is the space spanned by functions of $\tau$ of the form $\Psi_{a}(u ; \tau)$.

We also need genus-one two-point correlation functions. They are constructed from the $q_{\tau}$-traces of products or iterates of two geometricallymodified intertwining operators as follows: For $a_{1}, a_{2}, a_{3}, a_{4} \in \mathcal{A}, i=1, \ldots$, $N_{a_{1} a_{3}}^{a_{4}}, j=1, \ldots, N_{a_{2} a_{4}}^{a_{3}}$, consider

$$
\operatorname{Tr}_{W^{a_{4}}} \mathcal{Y}_{a_{1} a_{3} ; i}^{a_{4} ;(1)}\left(\mathcal{U}\left(e^{2 \pi i z_{1}}\right) w_{a_{1}}, e^{2 \pi i z_{1}}\right) \mathcal{Y}_{a_{2} a_{4} ; j}^{a_{3} ;(2)}\left(\mathcal{U}\left(e^{2 \pi i z_{2}}\right) w_{a_{2}}, e^{2 \pi i z_{2}}\right) q_{\tau}^{L(0)-\frac{c}{24}} .
$$

In [H7], it was shown that these $q_{\tau}$-traces are absolutely convergent when $0<\left|q_{\tau}\right|<\left|e^{2 \pi i z_{1}}\right|<\left|e^{2 \pi i z_{2}}\right|<1$ and can be analytically extended to multivalued analytic functions on

$$
M_{1}^{2}=\left\{\left(z_{1}, z_{2}, \tau\right) \in \mathbb{C}^{3} \mid z_{1} \neq z_{2}+p \tau+q \text { for } p, q \in \mathbb{Z}, \tau \in \mathbb{H}\right\} .
$$

(In fact, these multi-valued analytic functions depend only on $z_{1}-z_{2}$ and $\tau$ by the $L(-1)$-derivative property. See [H7].) These multi-valued analytic functions on $M_{1}^{2}$ are genus-one two-point correlation functions. They can be lifted to single-valued analytic functions on the universal covering $\widetilde{M}_{1}^{2}$. As in the case of genus-zero correlation functions, these liftings are not unique. To obtain a unique single-valued analytic function on $\widetilde{M}_{1}^{2}$ from a multi-valued analytic functions $f$ on $M_{1}^{2}$, we have to give a single valued branch of $f$ on some simply connected region in $M_{1}^{2}$ or values of $f$ on some simplyconnected subset of $M_{1}^{2}$. In fact, (2.5) gives a single valued branch of its analytic extension in the region given by $0<\left|q_{\tau}\right|<\left|e^{2 \pi i z_{1}}\right|<\left|e^{2 \pi i z_{2}}\right|<1$. We denote this single-valued analytic function on $\widetilde{M}_{1}^{2}$ by

$$
E\left(\operatorname{Tr}_{W^{a_{4}}} \mathcal{Y}_{a_{1} a_{3} ; i}^{a_{4} ;(1)}\left(\mathcal{U}\left(e^{2 \pi i z_{1}}\right) w_{a_{1}}, e^{2 \pi i z_{1}}\right) \mathcal{Y}_{a_{2} a_{4} ; j}^{a_{3} ;(2)}\left(\mathcal{U}\left(e^{2 \pi i z_{2}}\right) w_{a_{2}}, e^{2 \pi i z_{2}}\right) q_{\tau}^{L(0)-\frac{c}{24}}\right) .
$$

Similarly, these genus-one two-point correlation functions can also be constructed from $q_{\tau}$-traces of iterates of two intertwining operators as follows: For $a_{1}, a_{2}, a_{5}, a_{6} \in \mathcal{A}, k=1, \ldots, N_{a_{3} a_{4}}^{a_{4}}, l=1, \ldots, N_{a_{1} a_{2}}^{a_{3}}$, consider

$$
\operatorname{Tr}_{W^{a_{6}}} \mathcal{Y}_{a_{5} a_{6} ; k}^{a_{6} ;(3)}\left(\mathcal{U}\left(e^{2 \pi i z_{1}}\right) \mathcal{Y}_{a_{1} a_{2} ; l}^{a_{5} ;(4)}\left(w_{a_{1}}, z_{1}-z_{2}\right) w_{a_{2}}, e^{2 \pi i z_{2}}\right) q_{\tau}^{L(0)-\frac{c}{24}}
$$

In [H7], it was shown that these $q_{\tau}$-traces are absolutely convergent when $0<\left|q_{\tau}\right|<\left|e^{2 \pi i z_{2}}\right|<1$ and $0<\left|e^{2 \pi i\left(z_{1}-z_{2}\right)}-1\right|<1$ and can be analytically extended to multi-valued analytic functions on $M_{1}^{2}$. (Again, these multi-valued 
analytic functions depend only on $z_{1}-z_{2}$ and $\tau$ by the $L(-1)$-derivative property.) These multi-valued analytic functions on $M_{1}^{2}$ are also genus-one two-point correlation functions. The multi-valued analytic extension of (2.7) can also be lifted uniquely to a single-valued analytic function on $\widetilde{M}_{1}^{2}$ using the single-valued branch (2.7). We denote it by

$$
E\left(\operatorname{Tr}_{W^{a_{6}}} \mathcal{Y}_{a_{5} a_{6} ; k}^{a_{6} ;(3)}\left(\mathcal{U}\left(e^{2 \pi i z_{1}}\right) \mathcal{Y}_{a_{1} a_{2} ; l}^{a_{5} ;(4)}\left(w_{a_{1}}, z_{1}-z_{2}\right) w_{a_{2}}, e^{2 \pi i z_{2}}\right) q_{\tau}^{L(0)-\frac{c}{24}}\right) .
$$

In [H7], an associativity property for geometrically-modified intertwining operators is proved. This associativity together with the convergence property of $q_{\tau}$-traces of gives

$$
\begin{aligned}
& E\left(\operatorname{Tr}_{W^{a_{4}}} \mathcal{Y}_{a_{1} a_{3} ; i}^{a_{4} ;(1)}\left(\mathcal{U}\left(e^{2 \pi i z_{1}}\right) w_{a_{1}}, e^{2 \pi i z_{1}}\right) \mathcal{Y}_{a_{2} a_{4} ; j}^{a_{3} ;(2)}\left(\mathcal{U}\left(e^{2 \pi i z_{2}}\right) w_{a_{2}}, e^{2 \pi i z_{2}}\right) q_{\tau}^{L(0)-\frac{c}{24}}\right)
\end{aligned}
$$

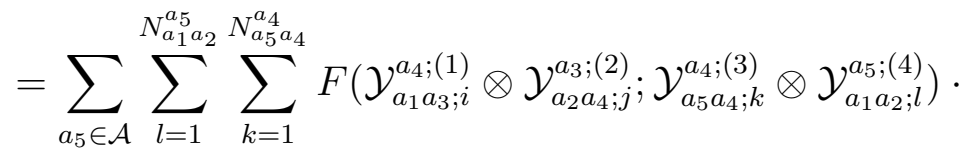

$$
\begin{aligned}
& \cdot E\left(\operatorname{Tr}_{W^{a_{4}}} \mathcal{Y}_{a_{5} a_{4} ; k}^{a_{4} ;(3)}\left(\mathcal{U}\left(e^{2 \pi i z_{1}}\right) \mathcal{Y}_{a_{1} a_{2} ; l}^{a_{5} ;(4)}\left(w_{a_{1}}, z_{1}-z_{2}\right) w_{a_{2}}, e^{2 \pi i z_{2}}\right) q_{\tau}^{L(0)}-\frac{c}{\left(2^{4}\right.} \cdot 9\right)
\end{aligned}
$$

and

$$
\begin{gathered}
E\left(\operatorname{Tr}_{W^{a_{4}}} \mathcal{Y}_{a_{3} a_{4} ; k}^{a_{4} ;(3)}\left(\mathcal{U}\left(e^{2 \pi i z_{1}}\right) \mathcal{Y}_{a_{1} a_{2} ; l}^{\left.a_{3} ; 4\right)}\left(w_{a_{1}}, z_{1}-z_{2}\right) w_{a_{2}}, e^{2 \pi i z_{2}}\right) q_{\tau}^{L(0)-\frac{c}{24}}\right. \\
=\sum_{a_{5} \in \mathcal{A}} \sum_{i=1}^{N_{a_{1} a_{2}}^{a_{2}}} \sum_{j=1}^{N_{a_{6} a_{3}}^{a_{4}}} F^{-1}\left(\mathcal{Y}_{a_{3} a_{4} ; k}^{a_{4} ;(3)} \otimes \mathcal{Y}_{a_{1} a_{2} ; l}^{a_{3} ;(4)} ; \mathcal{Y}_{a_{1} a_{5} ; i}^{a_{4} ;(1)} \otimes \mathcal{Y}_{a_{2} a_{4} ; j}^{a_{5} ;(2)}\right) \cdot \\
\cdot E\left(\operatorname{Tr}_{W^{a_{4}}{ }_{4}} \mathcal{Y}_{a_{1} a_{5} ; i}^{a_{4} ;(1)}\left(\mathcal{U}\left(e^{2 \pi i z_{1}}\right) w_{a_{1}}, e^{2 \pi i z_{1}}\right) \cdot\right. \\
\cdot \mathcal{Y}_{a_{2} a_{4} ; j}^{a_{5} ;(2)}\left(\mathcal{U}\left(e^{2 \pi i z_{2}}\right) w_{a_{2}}, e^{2 \pi i z_{2}}\right) q_{\tau}^{L(0)-\frac{c}{24}} \gamma_{2} .10
\end{gathered}
$$

In particular, the space of all single-valued analytic functions on $\widetilde{M}_{1}^{2}$ spanned by functions of the form (2.6) and the space of all single-valued analytic functions on $\widetilde{M}_{1}^{2}$ spanned by functions of the form (2.8) are the same. We shall denote this space by $\mathbb{G}_{1 ; 2}^{e}$.

We need the following result:

Proposition 2.2 For $a_{1}, a_{2} \in \mathcal{A}$, the maps from $W^{a_{1}} \otimes W^{a_{2}}$ to $\mathbb{G}_{1 ; 2}^{e}$ given by

$$
\begin{aligned}
& w_{a_{1}} \otimes w_{a_{2}} \mapsto \\
& \quad E\left(\operatorname{Tr}_{W^{a_{4}}} \mathcal{Y}_{a_{1} a_{3} ; i}^{a_{4} ;(1)}\left(\mathcal{U}\left(e^{2 \pi i z_{1}}\right) w_{a_{1}}, e^{2 \pi i z_{1}}\right) \mathcal{Y}_{a_{2} a_{4} ; j}^{a_{3} ;(2)}\left(\mathcal{U}\left(e^{2 \pi i z_{2}}\right) w_{a_{2}}, e^{2 \pi i z_{2}}\right) q_{\tau}^{L(0)-\frac{c}{24}}\right),
\end{aligned}
$$


$a_{3}, a_{4} \in \mathcal{A}, i=1, \ldots, N_{a_{1} a_{3}}^{a_{4}}, j=1, \ldots, N_{a_{2} a_{4}}^{a_{3}}$, are linearly independent. Similarly, for $a_{1}, a_{2} \in \mathcal{A}$, the maps from $W^{a_{1}} \otimes W^{a_{2}}$ to $\mathbb{G}_{1 ; 2}^{e}$ given by

$w_{a_{1}} \otimes w_{a_{2}} \mapsto E\left(\operatorname{Tr}_{W^{a_{4}}} \mathcal{Y}_{a_{3} a_{4} ; k}^{a_{4} ;(3)}\left(\mathcal{U}\left(e^{2 \pi i z_{2}}\right) \mathcal{Y}_{a_{1} a_{2} ; l}^{a_{3} ;(4)}\left(w_{a_{1}}, z_{1}-z_{2}\right) w_{a_{2}}, e^{2 \pi i z_{2}}\right) q_{\tau}^{L(0)-\frac{c}{24}}\right)$, $a_{3}, a_{4} \in \mathcal{A}, k=1, \ldots, N_{a_{3} a_{4}}^{a_{3}}, l=1, \ldots, N_{a_{1} a_{2}}^{a_{3}}$, are linearly independent.

Proof. We prove only the linear independence of the maps obtained from $q_{\tau}$-traces of iterates of intertwining operators. For the linear independence of the maps obtained from traces of products of intertwining operators, the proof is similar.

Since analytic extensions are unique, we need only prove that the maps given by

$$
w_{a_{1}} \otimes w_{a_{2}} \mapsto \operatorname{Tr}_{W^{a_{4}}} \mathcal{Y}_{a_{3} a_{4} ; k}^{a_{4} ;(3)}\left(\mathcal{U}\left(e^{2 \pi i z_{1}}\right) \mathcal{Y}_{a_{1} a_{2} ; l}^{a_{3} ;(4)}\left(w_{a_{1}}, z_{1}-z_{2}\right) w_{a_{2}}, e^{2 \pi i z_{2}}\right) q_{\tau}^{L(0)-\frac{c}{24}},
$$

$a_{3}, a_{4} \in \mathcal{A}, k=1, \ldots, N_{a_{3} a_{4}}^{a_{3}}, l=1, \ldots, N_{a_{1} a_{2}}^{a_{3}}$, are linearly independent. Assume

$$
\begin{aligned}
& \sum_{a_{3}, a_{4} \in \mathcal{A}} \sum_{k=1}^{N_{a_{3} a_{4}}^{a_{4}}} \sum_{l=1}^{N_{a_{1} a_{4}}^{a_{2}}} \lambda_{a_{3}, a_{4}, k, l} \\
& \quad=0 \\
& \quad \operatorname{Tr}_{W^{a_{4}} \mathcal{Y}_{a_{3} a_{4} ; k}} \mathcal{Y}^{a_{4} ;(3)}\left(\mathcal{U}\left(e^{2 \pi i z_{2}}\right) \mathcal{Y}_{a_{1} a_{2} ; l}^{a_{3} ;(4)}\left(w_{a_{1}}, z_{1}-z_{2}\right) w_{a_{2}}, e^{2 \pi i z_{2}}\right) q_{\tau}^{L(0)-\frac{c}{24}}
\end{aligned}
$$

for $w_{a_{1}} \in W^{a_{1}}$ and $w_{a_{2}} \in W^{a_{2}}$. Since (2.11) holds for all $z_{1}$ and $z_{2}$ satisfying $0<\left|q_{\tau}\right|<\left|e^{2 \pi i z_{2}}\right|<1$ and $0<\left|e^{2 \pi i\left(z_{1}-z_{2}\right)}-1\right|<1$, we obtain the following equation in which the variable $z_{1}-z_{2}$ is replaced by a formal variable $x_{0}$ :

$$
\begin{aligned}
& \sum_{a_{3}, a_{4} \in \mathcal{A}} \sum_{k=1}^{N_{a_{3} a_{4}}^{a_{4}}} \sum_{l=1}^{N_{a_{1} a_{4}}^{a_{2}}} \lambda_{a_{3}, a_{4}, k, l} \\
& \quad=0 \\
& \quad \operatorname{Tr}_{W^{a_{4}} \mathcal{Y}_{a_{3} a_{4} ; k}^{a_{4} ;(3)}\left(\mathcal{U}\left(e^{2 \pi i z_{2}}\right) \mathcal{Y}_{a_{1} a_{2} ; l}^{a_{3} ;(4)}\left(w_{a_{1}}, x_{0}\right) w_{a_{2}}, e^{2 \pi i z_{2}}\right) q_{\tau}^{L(0)-\frac{c}{24}}}
\end{aligned}
$$

for $w_{a_{1}} \in W^{a_{1}}$ and $w_{a_{2}} \in W^{a_{2}}$. We want to show that $\lambda_{a_{3}, a_{4}, k, l}=0$ for $a_{3}, a_{4} \in \mathcal{A}, k=1, \ldots, N_{a_{3} a_{4}}^{a_{3}}$ and $l=1, \ldots, N_{a_{1} a_{2}}^{a_{3}}$.

As in the proof of Proposition 1.2, there exists an isomorphism

$$
f: W^{a_{1}} \bigotimes_{P\left(z_{1}-z_{2}\right)} W^{a_{2}} \rightarrow \oplus_{a_{3} \in \mathcal{A}} N_{a_{1} a_{2}}^{a_{3}} W^{a_{3}}
$$


such that for $w_{a_{1}} \in W^{a_{1}}$ and $w_{a_{2}} \in W^{a_{2}}$,

$$
\bar{\pi}_{a_{3} ; l}\left(\bar{f}\left(w_{a_{1}} \otimes_{P\left(z_{z_{1}-z_{2}}\right)} w_{a_{2}}\right)\right)=\mathcal{Y}_{a_{1} a_{2} ; l}^{a_{3} ;(4)}\left(w_{a_{1}}, z_{1}-z_{2}\right) w_{a_{2}},
$$

where $\pi_{a_{3} ; l}$ is the projections from $\oplus_{a_{3} \in \mathcal{A}} N_{a_{1} a_{2}}^{a_{3}} W^{a_{3}}$ to the l-th copy of $W^{a_{3}}$ and

$$
\bar{\pi}_{a_{3} ; l}: \overline{\oplus_{a_{3} \in \mathcal{A}} N_{a_{1} a_{2}}^{a_{3}} W^{a_{3}}} \rightarrow \bar{W}^{a_{3}}
$$

and

$$
\bar{f}: \overline{W^{a_{1}} \bigotimes_{P\left(z_{1}-z_{2}\right)} W^{a_{2}}} \rightarrow \overline{\oplus_{a_{3} \in \mathcal{A}} N_{a_{1} a_{2}}^{a_{3} W^{a_{3}}}}
$$

are the natural extension of $\pi_{a_{3} ; l}$ and $f$, respectively. Let $\mathcal{Y}_{2}$ be the intertwining operator corresponding to the intertwining map $\bigotimes_{P\left(z_{2}\right)}: W^{a_{1}} \otimes W^{a_{2}} \rightarrow$ $W^{a_{1}} \bigotimes_{P\left(z_{1}-z_{2}\right)} W^{a_{2}}$ (see [HL1] and [HL4]). Then we have

$$
\pi_{a_{3} ; l}\left(f\left(\mathcal{Y}_{2}\left(w_{a_{1}}, x\right) w_{a_{2}}\right)\right)=\mathcal{Y}_{a_{1} a_{2} ; j}^{a_{3} ;(4)}\left(w_{a_{1}}, x\right) w_{a_{2}}
$$

for $w_{a_{1}} \in W^{a_{1}}$ and $w_{a_{2}} \in W^{a_{2}}$. For $a_{4} \in \mathcal{A}$, let $\mathcal{Y}_{a_{4}}$ be the intertwining

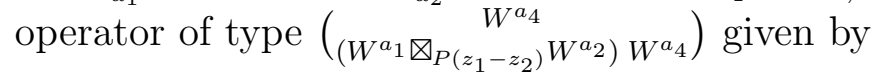

$$
\mathcal{Y}_{a_{4}}(w, x) w_{a_{4}}=\sum_{a_{3} \in \mathcal{A}} \sum_{k=1}^{N_{a_{3} a_{4}}^{a_{4}}} \sum_{l=1}^{N_{a_{1} a_{4}}^{a_{2}}} \lambda_{a_{3}, a_{4}, k, l} \mathcal{Y}_{a_{3} a_{4} ; k}^{a_{4} ;(3)}\left(\pi_{a_{3} ; l}(f(w)), x\right) w_{a_{4}}
$$

for $w \in W^{a_{1}} \bigotimes_{P\left(z_{1}-z_{2}\right)} W^{a_{2}}$ and $w_{a_{4}} \in W^{a_{4}}$. Then the left-hand side of (2.12) is equal to

$$
\begin{aligned}
& \sum_{a_{3}, a_{4} \in \mathcal{A}} \sum_{k=1}^{N_{a_{3} a_{4}}^{a_{4} a_{4}}} \sum_{l=1}^{N_{a_{1} a_{2}}^{a_{3}}} \lambda_{a_{3}, a_{4}, k, l} \\
& \quad \cdot \operatorname{Tr}_{W^{a_{4}}{ }} \mathcal{Y}_{a_{3} a_{4} ; k}^{a_{4} ;(3)}\left(\mathcal{U}\left(e^{2 \pi i z_{2}}\right) \pi_{a_{3} ; l}\left(f\left(\mathcal{Y}_{2}\left(w_{a_{1}}, x_{0}\right) w_{a_{2}}\right)\right), e^{2 \pi i z_{2}}\right) q_{\tau}^{L(0)-\frac{c}{24}} \\
& =\sum_{a_{4} \in \mathcal{A}} \operatorname{Tr}_{W^{a_{4}}} \mathcal{Y}_{a_{4}}\left(\mathcal{U}\left(e^{2 \pi i z_{2}}\right) \mathcal{Y}_{2}\left(w_{a_{1}}, x_{0}\right) w_{a_{2}}, e^{2 \pi i z_{2}}\right) q_{\tau}^{L(0)-\frac{c}{24}}
\end{aligned}
$$

By (2.12) and (2.14), we have

$$
\sum_{a_{4} \in \mathcal{A}} \operatorname{Tr}_{W^{a_{4}}} \mathcal{Y}_{a_{4}}\left(\mathcal{U}\left(e^{2 \pi i z_{2}}\right) \mathcal{Y}_{2}\left(w_{a_{1}}, x_{0}\right) w_{a_{2}}, e^{2 \pi i z_{2}}\right) q_{\tau}^{L(0)-\frac{c}{24}}=0
$$


for $w_{a_{1}} \in W^{a_{1}}$ and $w_{a_{2}} \in W^{a_{2}}$. Since the coefficients of $\mathcal{Y}_{2}\left(w_{a_{1}}, x_{0}\right) w_{a_{2}}$ for $w_{a_{1}} \in W^{a_{1}}$ and $w_{a_{2}} \in W^{a_{2}}$ span $W^{a_{1}} \bigotimes_{P\left(z_{1}-z_{2}\right)} W^{a_{2}}$, we obtain from (2.15) that

$$
\sum_{a_{4} \in \mathcal{A}} \operatorname{Tr}_{W^{a_{4}}} \mathcal{Y}_{a_{4}}\left(\mathcal{U}\left(e^{2 \pi i z_{2}}\right) w, e^{2 \pi i z_{2}}\right) q_{\tau}^{L(0)-\frac{c}{24}}=0
$$

for $w \in W^{a_{1}} \bigotimes_{P\left(z_{1}-z_{2}\right)} W^{a_{2}}$ and $w_{a_{3}} \in W^{a_{3}}$.

Since $W^{a_{4}}$ for $a_{4} \in \mathcal{A}$ are irreducible $V$-modules, we have $T\left(W^{a_{4}}\right)=$ $W_{\left(h_{a_{4}}\right)}^{a_{4}}$, where

$$
T\left(W^{a_{4}}\right)=\left\{w \in W^{a_{4}} \mid u_{n} w=0, u \in V, \text { wt } u-n-1<0\right\}
$$

(see [H7]). Since $\tau \in \mathbb{H}$ is arbitrary, from (2.16), we have

$$
\sum_{a_{4} \in \mathcal{A}} \operatorname{Tr}_{T\left(W^{a_{4}}\right)} O \mathcal{Y}_{a_{4}}(\mathcal{U}(1) w)=0
$$

for $w \in W^{a_{1}} \bigotimes_{P\left(z_{1}-z_{2}\right)} W^{a_{2}}$, where

$$
o \mathcal{Y}_{a_{4}}(\tilde{w})=\left(\mathcal{Y}_{a_{4}}\right)_{\mathrm{Wt} \hat{w}-1}(\hat{w})
$$

for homogeneous $\tilde{w} \in W^{a_{1}} \bigotimes_{P\left(z_{1}-z_{2}\right)} W^{a_{2}}$ (see Chapter 6 of [H7] for more details). Since $W^{a_{4}}$ for $a_{4} \in \mathcal{A}$ are inequivalent irreducible $V$-modules, $T\left(W^{a_{4}}\right)$ for $a_{4} \in \mathcal{A}$ are inequivalent irreducible $\tilde{A}(V)$-modules by Proposition 6.5 in [H7]. Thus $\operatorname{Tr}_{T\left(W^{a_{4}}\right)}$ for $a_{4} \in \mathcal{A}$ are linearly independent. So from (2.17), we obtain

$$
o_{\mathcal{Y}_{4}}(\mathcal{U}(1) w)=0
$$

for $w \in W^{a_{1}} \bigotimes_{P\left(z_{1}-z_{2}\right)} W^{a_{2}}$. Thus $\rho\left(\mathcal{Y}_{a_{4}}\right)=0$ where

$$
\rho\left(\mathcal{Y}_{a_{4}}\right): \tilde{A}\left(W^{a_{1}} \otimes_{P\left(z_{1}-z_{2}\right)} W^{a_{2}}\right) \otimes_{\tilde{A}(V)} T\left(W^{a_{4}}\right) \rightarrow T\left(W^{a_{4}}\right)
$$

is given by

$$
\rho\left(\mathcal{Y}_{a_{4}}\right)\left(\left(w+\tilde{O}\left(W^{a_{1}} \bigotimes_{P\left(z_{1}-z_{2}\right)} W^{a_{2}}\right)\right) \otimes w_{a_{4}}\right)=o \mathcal{Y}_{a_{4}}(\mathcal{U}(1) w) w_{a_{4}}
$$

for $w \in W^{a_{1}} \bigotimes_{P\left(z_{1}-z_{2}\right)} W^{a_{2}}$ and $w_{a_{4}} \in T\left(W^{a_{4}}\right)$ (see chapter 6 of [H7]). By Theorem 6.9 in [H7], $\rho\left(\mathcal{Y}_{a_{4}}\right)=0$ is equivalent to $\mathcal{Y}_{a_{4}}=0$ for $a_{4} \in \mathcal{A}$, that is

$$
\mathcal{Y}_{a_{4}}(w, x)=0
$$

for $a_{4} \in \mathcal{A}$ and $w \in W^{a_{1}} \bigotimes_{P\left(z_{1}-z_{2}\right)} W^{a_{2}}$. 
For $a_{3}, a_{4} \in \mathcal{A}$ and $l=1, \ldots, N_{a_{1} a_{2}}^{a_{3}}$, take $w$ to be an element of the tensor product module $W^{a_{1}} \bigotimes_{P\left(z_{1}-z_{2}\right)} W^{a_{2}}$ such that $f(w)$ is in the $l$-th copy of $W^{a_{3}}$ in $\oplus_{a_{3} \in \mathcal{A}} N_{a_{1} a_{2}}^{a_{3}} W^{a_{3}}$, that is, $\pi_{a_{3} ; l}(f(w))=f(w), \pi_{a_{3} ; m}(f(w))=0$ for $m \neq l$ and $\pi_{a ; m}(f(w))=0$ for $a \neq a_{3}$. Then by (2.19) and the definition of the intertwining operator $\mathcal{Y}_{a_{4}}$, we have

$$
\sum_{k=1}^{N_{a_{3} a_{4}}^{a_{4}}} \lambda_{a_{3}, a_{4}, k, l} \mathcal{Y}_{a_{3} a_{4} ; k}^{a_{4} ;(3)}(f(w), x)=0 .
$$

Since $f(w)$ is an arbitrary element of the $l$-th copy of $W^{a_{3}}$ in $\oplus_{a_{3} \in \mathcal{A}} N_{a_{1} a_{2}}^{a_{3}} W^{a_{3}}$, we obtain

$$
\sum_{k=1}^{N_{a_{3} a_{4}}^{a_{4}}} \lambda_{a_{3}, a_{4}, k, l} \mathcal{Y}_{a_{3} a_{4} ; k}^{a_{4} ;(3)}=0
$$

for $a_{3}, a_{4} \in \mathcal{A}$ and $l=1, \ldots, N_{a_{1} a_{2}}^{a_{3}}$. Since $\mathcal{Y}_{a_{3} a_{4} ; k}^{a_{4} ;(3)}$ for $k=1, \ldots, N_{a_{3} a_{4}}^{a_{4}}$ are linearly independent, we obtain $\lambda_{a_{3}, a_{4}, k, l}=0$ for $a_{3}, a_{4} \in \mathcal{A}, k=1, \ldots, N_{a_{3} a_{4}}^{a_{4}}$ and $l=1, \ldots, N_{a_{1} a_{2}}^{a_{3}}$.

We now introduce a space $\mathcal{F}_{1 ; 2}$ spanned by linear maps of the form

$$
\begin{aligned}
\Psi_{a_{1}, a_{2}, a_{3}}^{k, l}: \coprod_{a \in \mathcal{A}} W^{a} \otimes W^{a^{\prime}} & \rightarrow \mathbb{G}_{1 ; 2}^{e} \\
w_{a} \otimes w_{a^{\prime}} & \mapsto \Psi_{a_{1}, a_{2}, a_{3}}\left(w_{a}, w_{a^{\prime}} ; z_{1}, z_{2} ; \tau\right)
\end{aligned}
$$

for $a_{1}, a_{2}, a_{3} \in \mathcal{A}, k=1, \ldots, N_{a_{3} a_{1}}^{a_{1}}, l=1, \ldots, N_{a_{2} a_{2}^{\prime}}^{a_{3}}$, where

$$
\Psi_{a_{1}, a_{2}, a_{3}}^{k, l}\left(w_{a}, w_{a^{\prime}} ; z_{1}, z_{2} ; \tau\right)=0
$$

when $a \neq a_{2}$ and

$$
\begin{aligned}
& \Psi_{a_{1}, a_{2}, a_{3}}^{k, l}\left(w_{a_{2}}, w_{a_{2}^{\prime}} ; z_{1}, z_{2} ; \tau\right) \\
& \quad=E\left(\operatorname{Tr}_{W^{a_{1}}} \mathcal{Y}_{a_{3} a_{1} ; k}^{a_{1} ;(1)}\left(\mathcal{U}\left(e^{2 \pi i z_{2}}\right) \mathcal{Y}_{a_{2} a_{2}^{\prime} ; l}^{a_{3} ;(2)}\left(w_{a_{2}}, z_{1}-z_{2}\right) w_{a_{2}^{\prime}}, e^{2 \pi i z_{2}}\right) q_{\tau}^{L(0)-\frac{c}{24}}\right) .
\end{aligned}
$$

Let $\mathcal{F}_{1 ; 2}^{e}$ be the subspace of $\mathcal{F}_{1 ; 2}$ spanned by maps of the form $\Psi_{a_{1}, a_{2}, e}^{1,1}$ for $a_{1}, a_{2} \in \mathcal{A}$ and let $\mathcal{F}_{1 ; 2}^{n e}$ be the subspace of $F_{1 ; 2}$ spanned by maps of the form $\Psi_{a_{1}, a_{2}, a_{3}}^{k, l}$ for $a_{1}, a_{2}, a_{3} \in \mathcal{A}, a_{3} \neq e, k=1, \ldots, N_{a_{3} a_{1}}^{a_{1}}$ and $l=1, \ldots, N_{a_{2} a_{2}^{\prime}}^{a_{3}}$. We have: 
Proposition 2.3 The intersection of $\mathcal{F}_{1 ; 2}^{e}$ and $\mathcal{F}_{1 ; 2}^{n e}$ is 0 . In particular,

$$
F_{1 ; 2}=\mathcal{F}_{1 ; 2}^{e} \oplus \mathcal{F}_{1 ; 2}^{n e}
$$

and there exist a projection $\pi: \mathcal{F}_{1 ; 2} \rightarrow \mathcal{F}_{1 ; 2}^{e}$.

Proof. By Proposition 2.2, $\Psi_{a_{1}, a_{2}, a_{3}}^{k, l}$ for $a_{1}, a_{2}, a_{3} \in \mathcal{A}, k=1, \ldots, N_{a_{3} a_{1}}^{a_{1}}$ and $l=1, \ldots, N_{a_{2} a_{2}^{\prime}}^{a_{3}}$ are linearly independent. Thus the intersection of the space spanned by $\Psi_{a_{1}, a_{2}, e}^{1,1}$ for $a_{1}, a_{2} \in \mathcal{A}$ and the space spanned by $\Psi_{a_{1}, a_{2}, a_{3}}^{k, l}$ for $a_{1}, a_{2}, a_{3} \in \mathcal{A}, a_{3} \neq e, k=1, \ldots, N_{a_{3} a_{1}}^{a_{1}}$ and $l=1, \ldots, N_{a_{2} a_{2}^{\prime}}^{a_{3}}$ are 0 .

We define $S: \mathcal{F}_{1 ; 1}^{e} \rightarrow \mathcal{F}_{1 ; 1}^{e}$ as follows: For $a \in \mathcal{A}$, let

$$
\begin{aligned}
\left(S\left(\Psi_{a}\right)\right)(u ; \tau) & =\Psi_{a}\left(\left(-\frac{1}{\tau}\right)^{L(0)} u ;-\frac{1}{\tau}\right) \\
& =\operatorname{Tr}_{W^{a}} \mathcal{Y}_{e a ; 1}^{a ;(1)}\left(\mathcal{U}\left(e^{-2 \pi i \frac{z_{2}}{\tau}}\right)\left(-\frac{1}{\tau}\right)^{L(0)} u, e^{-2 \pi i \frac{z_{2}}{\tau}}\right) q_{-\frac{1}{\tau}}^{L(0)-\frac{c}{24}}
\end{aligned}
$$

Here we have used our convention that

$$
\left(-\frac{1}{\tau}\right)^{L(0)}=e^{\left(\log \left(-\frac{1}{\tau}\right)\right) L(0)} .
$$

Note that by the modular invariance of genus-one one-point functions proved in [M] and [H7], $S\left(\Psi_{a}\right)$ is indeed in $\mathcal{F}_{1 ; 1}^{e}$. Thus we do obtain maps $S: \mathcal{F}_{1 ; 1}^{e} \rightarrow$ $\mathcal{F}_{1 ; 1}^{e}$.

Now we define an action of the map $S$ on the space $\mathcal{F}_{1 ; 2}^{e}$ by

$$
\left(S\left(\Psi_{a_{1}, a_{2}, e}^{1,1}\right)\right)\left(w_{a}, w_{a^{\prime}} ; z_{1}, z_{2} ; \tau\right)=0
$$

when $a \neq a_{2}$ and

$$
\begin{aligned}
&\left(S\left(\Psi_{a_{1}, a_{2}, e}^{1,1}\right)\right)\left(w_{a_{2}}, w_{a_{2}^{\prime}} ; z_{1}, z_{2} ; \tau\right) \\
&=E\left(\operatorname { T r } _ { W ^ { a _ { 1 } } } \mathcal { Y } _ { e a _ { 1 } ; 1 } ^ { a _ { 1 } ; ( 1 ) } \left(\mathcal{U}\left(e^{-2 \pi i \frac{z_{2}}{\tau}}\right)\left(-\frac{1}{\tau}\right)^{L(0)} \cdot\right.\right. \\
&\left.\left.\quad \mathcal{Y}_{a_{2} a_{2}^{\prime} ; 1}^{e ;(2)}\left(w_{a_{2}}, z_{1}-z_{2}\right) w_{a_{2}^{\prime}}, e^{-2 \pi i \frac{z_{2}}{\tau}}\right) q_{-\frac{1}{\tau}}^{L(0)-\frac{c}{24}}\right)
\end{aligned}
$$




$$
\begin{aligned}
= & E\left(\operatorname { T r } _ { W ^ { a _ { 1 } } } \mathcal { Y } _ { e a _ { 1 } ; 1 } ^ { a _ { 1 } ; ( 1 ) } \left(\mathcal{U}\left(e^{-2 \pi i \frac{z_{2}}{\tau}}\right) \cdot\right.\right. \\
& \left.\cdot \mathcal{Y}_{a_{2} a_{2}^{\prime} ; 1}^{e ;(2)}\left(\left(-\frac{1}{\tau}\right)^{L(0)} w_{a_{2}},-\frac{1}{\tau} z_{1}-\left(-\frac{1}{\tau} z_{2}\right)\right)\left(-\frac{1}{\tau}\right)^{L(0)} w_{a_{2}^{\prime}}, e^{-2 \pi i \frac{z_{2}}{\tau}}\right) . \\
& \left.\cdot q_{-\frac{1}{\tau}}^{L(0)-\frac{c}{24}}\right) \\
= & \Psi_{a_{1}, a_{2}, e}^{1,1}\left(\left(-\frac{1}{\tau}\right)^{L(0)} w_{a},\left(-\frac{1}{\tau}\right)^{L(0)} w_{a^{\prime}} ;-\frac{1}{\tau} z_{1},-\frac{1}{\tau} z_{2} ;-\frac{1}{\tau}\right) .
\end{aligned}
$$

We shall also need the following result:

Proposition 2.4 For $a \in A, u \in V$, we have

$$
\begin{aligned}
& \operatorname{Tr}_{W^{a}} Y_{W^{a}}\left(\mathcal{U}\left(e^{2 \pi i z}\right) u, e^{2 \pi i z}\right) q_{\tau}^{L(0)-\frac{c}{24}} \\
& \left.\quad=\operatorname{Tr}_{W^{a_{4}^{\prime}}} Y_{W^{a^{\prime}}}\left(\mathcal{U}\left(e^{-2 \pi i z}\right) e^{\pi i L(0)} u, e^{-2 \pi i z}\right) q_{\tau}^{L(0)-\frac{c}{24}}\right) .
\end{aligned}
$$

For $a_{3}, a_{4} \in \mathcal{A}, i=1, \ldots, N_{a_{1} a_{3}}^{a_{4}}, j=1, \ldots, N_{a_{2} a_{4}}^{a_{3}}, w_{a_{1}} \in W^{a_{1}}, w_{a_{2}} \in W^{a_{2}}$, we have

$$
\begin{aligned}
& E\left(\operatorname{Tr}_{W^{a_{4}}} \sigma_{23}\left(\mathcal{Y}_{a_{1} a_{4}^{\prime} ; i}^{\left.a_{3}^{\prime} ; 1\right)}\right)\left(\mathcal{U}\left(e^{2 \pi i z_{1}}\right) w_{a_{1}}, e^{2 \pi i z_{1}}\right)\right. \\
& \left.\cdot \sigma_{23}\left(\mathcal{Y}_{a_{2} a_{3}^{\prime} ; j}^{a_{4}^{\prime} ;(2)}\right)\left(\mathcal{U}\left(e^{2 \pi i z_{2}}\right) w_{a_{2}}, e^{2 \pi i z_{2}}\right) q_{\tau}^{L(0)-\frac{c}{24}}\right) \\
& =e^{-\pi i\left(h_{a_{1}}+h_{a_{2}}\right)} E\left(\operatorname{Tr}_{W^{a_{4}^{\prime}}} \mathcal{Y}_{a_{2} a_{3}^{\prime} ; j}^{a_{4}^{\prime} ;(2)}\left(\mathcal{U}\left(e^{-2 \pi i z_{2}}\right) e^{\pi i L(0)} w_{a_{2}}, e^{-2 \pi i z_{2}}\right)\right. \text {. } \\
& \left.\cdot \mathcal{Y}_{a_{1} a_{4}^{\prime} ; i}^{a_{3}^{\prime} ;(1)}\left(\mathcal{U}\left(e^{-2 \pi i z_{1}}\right) e^{\pi i L(0)} w_{a_{1}}, e^{-2 \pi i z_{1}}\right) q_{\tau}^{L(0)-\frac{c}{24}}\right)
\end{aligned}
$$

and

$$
\begin{aligned}
& E\left(\operatorname{Tr}_{W^{a_{4}^{\prime}}} \sigma_{23}\left(\mathcal{Y}_{a_{3} a_{4} ; k}^{a_{4} ;(3)}\right)\left(\mathcal{U}\left(e^{2 \pi i z_{2}}\right) \mathcal{Y}_{a_{1} a_{2} ; l}^{a_{3} ;(4)}\left(w_{a_{1}}, z_{1}-z_{2}\right) w_{a_{2}}, e^{2 \pi i z_{2}}\right) q_{\tau}^{L(0)-\frac{c}{24}}\right) \\
& =e^{\pi i h_{a_{3}}} E\left(\operatorname { T r } _ { W ^ { a _ { 4 } } } \mathcal { Y } _ { a _ { 3 } a _ { 4 } ; k } ^ { a _ { 4 } ; ( 3 ) } \left(\mathcal{U}\left(e^{2 \pi i z_{2}}\right) e^{-\pi i L(0)}\right.\right. \text {. } \\
& \left.\left.\cdot \mathcal{Y}_{a_{1} a_{2} ; l}^{a_{3} ;(4)}\left(w_{a_{1}}, z_{1}-z_{2}\right) w_{a_{2}}, e^{2 \pi i z_{2}}\right) q_{\tau}^{L(0)-\frac{c}{24}}\right) .
\end{aligned}
$$

Proof. These formulas follows immediately from (2.2). 


\section{Properties of fusing and braiding matrices}

In the present section, we prove some properties of the fusing and braiding matrices. These properties play important role in the proofs of Moore-Seiberg formulas in the next section and in the proof of the symmetry of the matrix associated to the modular transformation $\tau \mapsto-1 / \tau$ in Section 5 .

In this section, for $p=1,2,3,4,5,6$ and $a_{1}, a_{2}, a_{3} \in \mathcal{A}, \mathcal{Y}_{a_{1} a_{2} ; i}^{\left.a_{3} ; p\right)}, i=$ $1, \ldots, N_{a_{1} a_{2}}^{a_{3}}$, are bases of $\mathcal{V}_{a_{1} a_{2}}^{a_{3}}$.

Proposition 3.1 The following equality expressing the squares of braiding matrices in terms of the fusing matrices and the inverses of fusing matrices holds:

$$
\begin{gathered}
\sum_{a_{7} \in \mathcal{A}} \sum_{k=1}^{N_{a_{1} a_{2}}^{a_{7}}} \sum_{l=1}^{N_{a_{7} a_{3}}^{a_{4}}} F\left(\mathcal{Y}_{a_{1} a_{5} ; i}^{a_{4} ;(1)} \otimes \mathcal{Y}_{a_{2} a_{3} ; j}^{a_{5} ;(2)} ; \mathcal{Y}_{a_{7} a_{3} ; l}^{a_{4} ;(5)} \otimes \mathcal{Y}_{a_{1} a_{2} ; k}^{a_{7} ;(6)}\right) \\
\cdot e^{2(2 r+1) \pi i\left(h_{a_{7}}-h_{a_{1}}-h_{a_{2}}\right)} F^{-1}\left(\mathcal{Y}_{a_{7} a_{3} ; l}^{a_{4} ;(5)} \otimes \mathcal{Y}_{a_{1} a_{2} ; k}^{a_{7} ;(6)} ; \mathcal{Y}_{a_{1} a_{6} ; p}^{a_{4} ;(3)} \otimes \mathcal{Y}_{a_{2} a_{3} ; q}^{a_{6} ;(4)}\right) \\
=\left(B^{(r)}\right)^{2}\left(\mathcal{Y}_{a_{1} a_{5} ; i}^{a_{4} ;(1)} \otimes \mathcal{Y}_{a_{2} a_{3} ; j}^{a_{5} ;(2)} ; \mathcal{Y}_{a_{1} a_{6} ; p}^{a_{4} ;(3)} \otimes \mathcal{Y}_{a_{2} a_{3} ; q}^{a_{6} ;(4)}\right) .
\end{gathered}
$$

Proof. Let $w_{a_{1}} \in W^{a_{1}}, w_{a_{2}} \in W^{a_{2}}, w_{a_{3}} \in W^{a_{3}}$ and $w_{a_{4}^{\prime}} \in W^{a_{4}^{\prime}}$. Then we have

$$
\begin{gathered}
E\left(\left\langle w_{a_{4}^{\prime}}, \mathcal{Y}_{a_{1} a_{5} ; i}^{a_{4} ;(1)}\left(w_{a_{1}}, z_{1}\right) \mathcal{Y}_{a_{2} a_{3} ; j}^{a_{5} ;(2)}\left(w_{a_{2}}, z_{2}\right) w_{a_{3}}\right\rangle\right) \\
=\sum_{a_{7} \in \mathcal{A}} \sum_{k=1}^{N_{a_{1} a_{2}}^{a_{7}}} \sum_{l=1}^{N_{a_{7} a_{3}}^{a_{4}}} F\left(\mathcal{Y}_{a_{1} a_{5} ; i}^{a_{4} ;(1)} \otimes \mathcal{Y}_{a_{2} a_{3} ; j}^{a_{5} ;(2)} ; \mathcal{Y}_{a_{7} a_{3} ; l}^{a_{4} ;(5)} \otimes \mathcal{Y}_{a_{1} a_{2} ; k}^{a_{7} ;(6)}\right) . \\
\cdot E\left(\left\langle w_{a_{4}^{\prime}}, \mathcal{Y}_{a_{7} a_{3} ; l}^{a_{4} ;(5)}\left(\mathcal{Y}_{a_{1} a_{2} ; k}^{a_{7} ;(6)}\left(w_{a_{1}}, z_{1}-z_{2}\right) w_{a_{2}}, z_{2}\right) w_{a_{3}}\right\rangle\right) .
\end{gathered}
$$

Applying $\left(B^{(r)}\right)^{2}$ to both sides of the above formula, we obtain

$$
\begin{aligned}
&\left(B^{(r)}\right)^{2}\left(E\left(\left\langle w_{a_{4}^{\prime}}, \mathcal{Y}_{a_{1} a_{5} ; i}^{a_{4} ;(1)}\left(w_{a_{1}}, z_{1}\right) \mathcal{Y}_{a_{2} a_{3} ; j}^{a_{5} ;(2)}\left(w_{a_{2}}, z_{2}\right) w_{a_{3}}\right\rangle\right)\right) \\
&=\sum_{a_{7} \in \mathcal{A}} \sum_{k=1}^{N_{a_{1} a_{2}}^{a_{1}}} \sum_{l=1}^{N_{a_{7} a_{3}}^{a_{4}}} F\left(\mathcal{Y}_{a_{1} a_{5} ; i}^{a_{4} ;(1)} \otimes \mathcal{Y}_{a_{2} a_{3} ; j}^{a_{5} ;(2)} ; \mathcal{Y}_{a_{7} a_{3} ; l}^{a_{4} ;(5)} \otimes \mathcal{Y}_{a_{1} a_{2} ; k}^{a_{7} ;(6)}\right) . \\
& \cdot\left(B^{(r)}\right)^{2}\left(E\left(\left\langle w_{a_{4}^{\prime}}, \mathcal{Y}_{a_{7} a_{3} ; l}^{a_{4} ;(5)}\left(\mathcal{Y}_{a_{1} a_{2} ; k}^{a_{7} ;(6)}\left(w_{a_{1}}, z_{1}-z_{2}\right) w_{a_{2}}, z_{2}\right) w_{a_{3}}\right\rangle\right)\right) .
\end{aligned}
$$


In Section 1, we have seen that $\left(B^{(r)}\right)^{2}$ is the monodromy given by

$$
\log \left(z_{1}-z_{2}\right) \mapsto \log \left(z_{1}-z_{2}\right)+2(2 r+1) \pi i .
$$

Using this fact and

$$
\begin{aligned}
& \left.\mathcal{Y}_{a_{1} a_{2} ; k}^{a_{7} ;(6)}\left(w_{a_{1}}, x\right)\right|_{x^{n}=e^{n\left(\log \left(z_{1}-z_{2}\right)+2(2 r+1) \pi i\right)}, n \in \mathbb{C}} \\
& \quad=\left.e^{2(2 r+1) \pi i\left(h_{a_{7}}-h_{a_{1}}-h_{a_{2}}\right)} \mathcal{Y}_{a_{1} a_{2} ; k}^{a_{7} ;(6)}\left(w_{a_{1}}, x\right)\right|_{x^{n}=e^{n \log \left(z_{1}-z_{2}\right)}, n \in \mathbb{C}},
\end{aligned}
$$

we have

$$
\begin{aligned}
& \left(B^{(r)}\right)^{2}\left(E\left(\left\langle w_{a_{4}^{\prime}}, \mathcal{Y}_{a_{7} a_{3} ; l}^{a_{4} ;(5)}\left(\mathcal{Y}_{a_{1} a_{2} ; k}^{\left.a_{7} ; 6\right)}\left(w_{a_{1}}, z_{1}-z_{2}\right) w_{a_{2}}, z_{2}\right) w_{a_{3}}\right\rangle\right)\right) \\
& \quad=e^{2(2 r+1) \pi i\left(h_{a_{7}}-h_{a_{1}}-h_{a_{2}}\right)} E\left(\left\langle w_{a_{4}^{\prime}}, \mathcal{Y}_{a_{7} a_{3} ; l}^{a_{4} ;(5)}\left(\mathcal{Y}_{a_{1} a_{2} ; k}^{a_{7} ;(6)}\left(w_{a_{1}}, z_{1}-z_{2}\right) w_{a_{2}}, z_{2}\right) w_{a_{3}}\right\rangle\right) .
\end{aligned}
$$

Using (3.2), (3.3) and the associativity (1.6) expressed in terms of the matrix elements of the inverse of the fusing isomorphism, we obtain

$$
\begin{aligned}
& \left(B^{(r)}\right)^{2}\left(E\left(\left\langle w_{a_{4}^{\prime}}, \mathcal{Y}_{a_{1} a_{5} ; i}^{a_{4} ;(1)}\left(w_{a_{1}}, z_{1}\right) \mathcal{Y}_{a_{2} a_{3} ; j}^{a_{5} ;(2)}\left(w_{a_{2}}, z_{2}\right) w_{a_{3}}\right\rangle\right)\right)
\end{aligned}
$$

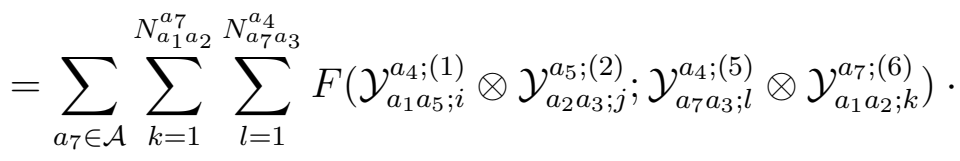

$$
\begin{aligned}
& \cdot e^{2(2 r+1) \pi i\left(h_{a_{7}}-h_{a_{1}}-h_{a_{2}}\right)} \text {. } \\
& \cdot E\left(\left\langle w_{a_{4}^{\prime}}, \mathcal{Y}_{a_{7} a_{3} ; l}^{a_{4} ;(5)}\left(\mathcal{Y}_{a_{1} a_{2} ; k}^{a_{7} ;(6)}\left(w_{a_{1}}, z_{1}-z_{2}\right) w_{a_{2}}, z_{2}\right) w_{a_{3}}\right\rangle\right)
\end{aligned}
$$

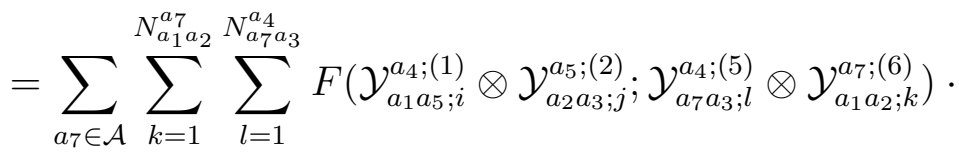

$$
\begin{aligned}
& \cdot e^{2(2 r+1) \pi i\left(h_{a_{7}}-h_{a_{1}}-h_{a_{2}}\right)} \text {. } \\
& \cdot \sum_{a_{6} \in \mathcal{A}} \sum_{p=1}^{N_{a_{1} a_{6}}^{a_{4}}} \sum_{q=1}^{N_{a_{2} a_{3}}^{a_{6}}} F^{-1}\left(\mathcal{Y}_{a_{7} a_{3} ; l}^{a_{4} ;(5)} \otimes \mathcal{Y}_{a_{1} a_{2} ; k}^{a_{7} ;(6)} ; \mathcal{Y}_{a_{1} a_{6} ; p}^{a_{4} ;(3)} \otimes \mathcal{Y}_{a_{2} a_{3} ; q}^{a_{6} ;(4)}\right) \text {. } \\
& \cdot E\left(\left\langle w_{a_{4}^{\prime}}, \mathcal{Y}_{a_{1} a_{6} ; p}^{a_{4} ;(3)}\left(w_{a_{1}}, z_{1}\right) \mathcal{Y}_{a_{2} a_{3} ; q}^{a_{6} ;(4)}\left(w_{a_{2}}, z_{2}\right) w_{a_{3}}\right\rangle\right) .
\end{aligned}
$$

Comparing (3.4) with the definition (1.15) of the matrix elements of $\left(B^{(r)}\right)^{2}$ and using Proposition 1.2, we obtain (3.1). 
Proposition 3.2 The following equality expressing the inverses of the fusing matrices in terms of the fusing matrices holds:

$$
\begin{aligned}
& F^{-1}\left(\mathcal{Y}_{a_{6} a_{3} ; l}^{a_{4} ;(1)} \otimes \mathcal{Y}_{a_{1} a_{2} ; k}^{a_{6} ;(2)} ; \mathcal{Y}_{a_{1} a_{5} ; i}^{a_{4} ;(3)} \otimes \mathcal{Y}_{a_{2} a_{3} ; j}^{a_{5}(4)}\right) \\
& \quad=F\left(\sigma_{12}\left(\mathcal{Y}_{a_{6} a_{3} ; l}^{a_{4} ;(1)}\right) \otimes \sigma_{12}\left(\mathcal{Y}_{a_{1} a_{2} ; k}^{a_{6} ;(2)}\right) ; \sigma_{12}\left(\mathcal{Y}_{a_{1} a_{5} ; i}^{a_{4} ;(3)}\right) \otimes \sigma_{12}\left(\mathcal{Y}_{a_{2} a_{3} ; j}^{a_{5} ;(4)}\right)\right)
\end{aligned}
$$

Proof. Let $w_{a_{1}} \in W^{a_{1}}, w_{a_{2}} \in W^{a_{2}}, w_{a_{3}} \in W^{a_{3}}$ and $w_{a_{4}^{\prime}} \in W^{a_{4}^{\prime}}$. Then using the definition of $\sigma_{12}$, the relation $\sigma_{12}^{2}=1$, the associativity (1.5) and the $L(-1)$-conjugation property (see [FHL]), we have

$$
\begin{aligned}
& E\left(\left\langle w_{a_{4}^{\prime}}, \mathcal{Y}_{a_{6} a_{3} ; l}^{a_{4} ;(1)}\left(\mathcal{Y}_{a_{1} a_{2} ; k}^{a_{6} ;(2)}\left(w_{a_{1}}, z_{1}-z_{2}\right) w_{a_{2}}, z_{2}\right) w_{a_{3}}\right\rangle\right) \\
& =E\left(\left\langle w_{a_{4}^{\prime}}, \sigma_{12}^{2}\left(\mathcal{Y}_{a_{6} a_{3} ; l}^{a_{4} ;()}\right)\left(\sigma_{12}^{2}\left(\mathcal{Y}_{a_{1} a_{2} ; k}^{a_{6} ;(2)}\right)\left(w_{a_{1}}, z_{1}-z_{2}\right) w_{a_{2}}, z_{2}\right) w_{a_{3}}\right\rangle\right) \\
& =e^{\pi i\left(h_{a_{4}}-h_{a_{6}}-h_{a_{3}}\right)} e^{\pi i\left(h_{a_{6}}-h_{a_{1}}-h_{a_{2}}\right)} \text {. } \\
& \cdot E\left(\left\langlew_{a_{4}^{\prime}}, e^{z_{2} L(-1)} \sigma_{12}\left(\mathcal{Y}_{a_{6} a_{3} ; l}^{a_{4} ;(1)}\right)\left(w_{a_{3}}, e^{-\pi i} z_{2}\right) \cdot\right.\right. \\
& \left.\left.\cdot e^{\left(z_{1}-z_{2}\right) L(-1)} \sigma_{12}\left(\mathcal{Y}_{a_{1} a_{2} ; k}^{a_{6} ;(2)}\right)\left(w_{a_{2}}, e^{-\pi i}\left(z_{1}-z_{2}\right)\right) w_{a_{1}}\right\rangle\right) \\
& =e^{\pi i\left(h_{a_{4}}-h_{a_{1}}-h_{a_{2}}-h_{a_{3}}\right)} E\left(\left\langlew_{a_{4}^{\prime}}, e^{z_{1} L(-1)} \sigma_{12}\left(\mathcal{Y}_{a_{6} a_{3} ; l}^{a_{4} ;(1)}\right)\left(w_{a_{3}}, e^{-\pi i} z_{1}\right)\right.\right. \text {. } \\
& \left.\left.\cdot \sigma_{12}\left(\mathcal{Y}_{a_{1} a_{2} ; k}^{a_{6}(2)}\right)\left(w_{a_{2}}, e^{-\pi i}\left(z_{1}-z_{2}\right)\right) w_{a_{1}}\right\rangle\right) \\
& =e^{\pi i\left(h_{a_{4}}-h_{a_{1}}-h_{a_{2}}-h_{a_{3}}\right)} \sum_{a_{5} \in \mathcal{A}} \sum_{i=1}^{N_{a_{1} a_{5}}^{a_{4}}} \sum_{j=1}^{N_{a_{2} a_{3}}^{a_{5}}} \\
& \cdot F\left(\sigma_{12}\left(\mathcal{Y}_{a_{6} a_{3} ; l}^{a_{4} ;(1)}\right) \otimes \sigma_{12}\left(\mathcal{Y}_{a_{1} a_{2} ; k}^{a_{6} ;(2)}\right) ; \sigma_{12}\left(\mathcal{Y}_{a_{1} a_{5} ; i}^{a_{4} ;(3)}\right) \otimes \sigma_{12}\left(\mathcal{Y}_{a_{2} a_{3} ; j}^{a_{5} ;(4)}\right)\right) \text {. } \\
& \cdot E\left(\left\langlew_{a_{4}^{\prime}}, e^{z_{1} L(-1)} \sigma_{12}\left(\mathcal{Y}_{a_{1} a_{5} ; i}^{a_{4} ;(3)}\right)\left(\sigma_{12}\left(\mathcal{Y}_{a_{2} a_{3} ; j}^{a_{5} ;(4)}\right)\left(w_{a_{3}}, e^{-\pi i} z_{2}\right) \cdot\right.\right.\right. \\
& \left.\left.\left.\cdot w_{a_{2}}, e^{-\pi i}\left(z_{1}-z_{2}\right)\right) w_{a_{1}}\right\rangle\right) \\
& =e^{\pi i\left(h_{a_{4}}-h_{a_{1}}-h_{a_{5}}\right)} e^{\pi i\left(h_{a_{5}}-h_{a_{2}}-h_{a_{3}}\right)} \sum_{a_{5} \in \mathcal{A}} \sum_{i=1}^{N_{a_{1} a_{5}}^{a_{4}}} \sum_{j=1}^{N_{a_{2} a_{3}}^{a_{5}}} \\
& \cdot F\left(\sigma_{12}\left(\mathcal{Y}_{a_{6} a_{3} ; l}^{a_{4} ;(1)}\right) \otimes \sigma_{12}\left(\mathcal{Y}_{a_{1} a_{2} ; k}^{a_{6} ;(2)}\right) ; \sigma_{12}\left(\mathcal{Y}_{a_{1} a_{5} ; i}^{a_{4} ;(3)}\right) \otimes \sigma_{12}\left(\mathcal{Y}_{a_{2} a_{3} ; j}^{a_{5} ;(4)}\right)\right) \text {. } \\
& \cdot E\left(\left\langlew_{a_{4}^{\prime}}, e^{z_{1} L(-1)} \sigma_{12}\left(\mathcal{Y}_{a_{1} a_{5} ; i}^{a_{4} ;(3)}\right)\left(e^{z_{2} L(-1)}\right. \text {. }\right.\right. \\
& \left.\left.\left.\cdot \sigma_{12}\left(\mathcal{Y}_{a_{2} a_{3} ; j}^{a_{5} ;(4)}\right)\left(w_{a_{3}}, e^{-\pi i} z_{2}\right) w_{a_{2}}, e^{-\pi i} z_{1}\right) w_{a_{1}}\right\rangle\right) \\
& =\sum_{a_{5} \in \mathcal{A}} \sum_{i=1}^{N_{a_{1} a_{5}}^{a_{4}}} \sum_{j=1}^{N_{a_{2} a_{3}}^{a_{5}}}
\end{aligned}
$$




$$
\begin{aligned}
& F\left(\sigma_{12}\left(\mathcal{Y}_{a_{6} a_{3} ; l}^{a_{4} ;(1)}\right) \otimes \sigma_{12}\left(\mathcal{Y}_{a_{1} a_{2} ; k}^{a_{6} ;(2)}\right) ; \sigma_{12}\left(\mathcal{Y}_{a_{1} a_{5} ; i}^{\left.a_{4} ; 3\right)}\right) \otimes \sigma_{12}\left(\mathcal{Y}_{a_{2} a_{3} ; j}^{a_{5} ;(4)}\right)\right) . \\
& \cdot E\left(\left\langle w_{a_{4}^{\prime}}, \mathcal{Y}_{a_{1} a_{5} ; i}^{a_{4} ;(3)}\left(w_{a_{1}}, z_{1}\right) \mathcal{Y}_{a_{2} a_{3} ; j}^{a_{5} ;(4)}\left(w_{a_{2}}, z_{2}\right) w_{a_{3}}\right\rangle\right) .
\end{aligned}
$$

Comparing (3.6) with (1.14) and using Proposition 1.2, we obtain (3.5).

In the proof of the next property of fusing matrices, we need the following lemma:

Lemma 3.3 For any $z_{1}, z_{2} \in \mathbb{C}^{\times}$satisfying $z_{1} \neq z_{2}$ and any $V$-module $W$, the following equalities for maps from $W$ to $\bar{W}$ holds:

$$
\begin{gathered}
e^{z_{2} L(1)} e^{z_{1}^{-1} L(-1)}=e^{\left(z_{1}-z_{2}\right)^{-1} L(-1)} e^{z_{1}^{-1} z_{2}\left(z_{1}-z_{2}\right) L(1)}\left(z_{1}\left(z_{1}-z_{2}\right)^{-1}\right)^{2 L(0)}, \\
e^{z_{1}^{-1} z_{2}\left(z_{1}-z_{2}\right) L(1)}\left(z_{1}\left(z_{1}-z_{2}\right)^{-1}\right)^{2 L(0)} e^{z_{1} L(1)}\left(e^{-\pi i} z_{1}^{-2}\right)^{L(0)} \\
=e^{\left(z_{1}-z_{2}\right) L(1)}\left(e^{-\pi i}\left(z_{1}-z_{2}\right)^{-2}\right)^{L(0)}
\end{gathered}
$$

Proof. From the identity

$\frac{1}{x^{-1}+z_{2}}-z_{1}^{-1}=\left(z_{1}\left(z_{1}-z_{2}\right)^{-1}\right)^{-2}\left(\frac{1}{\left(x-\left(z_{1}-z_{2}\right)^{-1}\right)^{-1}+z_{1}^{-1} z_{2}\left(z_{1}-z_{2}\right)}\right)$,

we obtain

$$
e^{-z_{2} x^{2} \frac{d}{d x}} e^{-z_{1}^{-1} \frac{d}{d x}} x=e^{-\left(z_{1}-z_{2}\right)^{-1} \frac{d}{d x}} e^{-z_{1}^{-1} z_{2}\left(z_{1}-z_{2}\right) x^{2} \frac{d}{d x}}\left(z_{1}\left(z_{1}-z_{2}\right)^{-1}\right)^{-2 x \frac{d}{d x}} x .
$$

Using (3.9) and the theory developed in Chapters 4 and 5 of [H3], we obtain (3.7).

To prove (3.8), we note that the weight of $L(1)$ is -1 . So we have

$$
\left(z_{1}\left(z_{1}-z_{2}\right)^{-1}\right)^{2 L(0)} L(1)\left(z_{1}\left(z_{1}-z_{2}\right)^{-1}\right)^{-2 L(0)}=z_{1}^{-2}\left(z_{1}-z_{2}\right)^{2} L(1) .
$$

Using (3.10), we obtain

$$
\begin{aligned}
& \left(z_{1}\left(z_{1}-z_{2}\right)^{-1}\right)^{2 L(0)} e^{z_{1} L(1)} \\
& \quad=\left(z_{1}\left(z_{1}-z_{2}\right)^{-1}\right)^{2 L(0)} e^{z_{1} L(1)}\left(z_{1}\left(z_{1}-z_{2}\right)^{-1}\right)^{-2 L(0)}\left(z_{1}\left(z_{1}-z_{2}\right)^{-1}\right)^{2 L(0)} \\
& \quad=e^{z_{1}\left(z_{1}\left(z_{1}-z_{2}\right)^{-1}\right)^{2 L(0)} L(1)\left(z_{1}\left(z_{1}-z_{2}\right)^{-1}\right)^{-2 L(0)}}\left(z_{1}\left(z_{1}-z_{2}\right)^{-1}\right)^{2 L(0)} \\
& \quad=e^{z_{1}^{-1}\left(z_{1}-z_{2}\right)^{2} L(1)}\left(z_{1}\left(z_{1}-z_{2}\right)^{-1}\right)^{2 L(0)}
\end{aligned}
$$

The equality (3.8) follows immediately from (3.11).

Let $\sigma_{123}=\sigma_{12} \sigma_{23}$ and $\sigma_{132}=\sigma_{23} \sigma_{12}$. We have: 
Proposition 3.4 The following equality between fusing matrices holds:

$$
\begin{aligned}
& F\left(\mathcal{Y}_{a_{1} a_{5} ; i}^{a_{4} ;(1)} \otimes \mathcal{Y}_{a_{2} a_{3} ; j}^{a_{5}(2)} ; \mathcal{Y}_{a_{6} a_{3} ; l}^{a_{4} ;(3)} \otimes \mathcal{Y}_{a_{1} a_{2} ; k}^{a_{6} ;(4)}\right) \\
& \quad=F\left(\sigma_{132}\left(\mathcal{Y}_{a_{2} a_{3} ; j}^{a_{5} ;(2)}\right) \otimes \sigma_{123}\left(\mathcal{Y}_{a_{1} a_{5} ; i}^{a_{4} ;(1)}\right) ; \sigma_{123}\left(\mathcal{Y}_{a_{1} a_{2} ; k}^{a_{6} ;(4)}\right) \otimes \sigma_{132}\left(\mathcal{Y}_{a_{6} a_{3} ; l}^{a_{4} ;(3)}\right)\right) .
\end{aligned}
$$

Proof. Let $w_{a_{1}} \in W^{a_{1}}, w_{a_{2}} \in W^{a_{2}}, w_{a_{3}} \in W^{a_{3}}$ and $w_{a_{4}^{\prime}} \in W^{a_{4}^{\prime}}$. Then using the definitions of $\sigma_{12}$ and $\sigma_{23}$ and the relations $\sigma_{12}^{2}=\sigma_{23}^{2}=1$, we obtain

$$
\begin{aligned}
& E\left(\left\langle w_{a_{4}^{\prime}}, \mathcal{Y}_{a_{1} a_{5} ; i}^{a_{4} ;(1)}\left(w_{a_{1}}, z_{1}\right) \mathcal{Y}_{a_{2} a_{3} ; j}^{a_{5} ;(2)}\left(w_{a_{2}}, z_{2}\right) w_{a_{3}}\right\rangle\right) \\
& =E\left(\left\langle w_{a_{4}^{\prime}}, \sigma_{23}^{2}\left(\mathcal{Y}_{a_{1} a_{5} ; i}^{a_{4} ;(1)}\right)\left(w_{a_{1}}, z_{1}\right) \sigma_{12}^{2}\left(\mathcal{Y}_{a_{2} a_{3} ; j}^{a_{5} ;(2)}\right)\left(w_{a_{2}}, z_{2}\right) w_{a_{3}}\right\rangle\right) \\
& =e^{\pi i\left(h_{a_{1}}+h_{a_{5}}-h_{a_{2}}-h_{a_{3}}\right)} \text {. } \\
& \cdot E\left(\left\langle\sigma_{23}\left(\mathcal{Y}_{a_{1} a_{5} ; i}^{a_{4} ;(1)}\right)\left(e^{z_{1} L(1)}\left(e^{-\pi i} z_{1}^{-2}\right)^{L(0)} w_{a_{1}}, z_{1}^{-1}\right) w_{a_{4}^{\prime}},\right.\right. \\
& \left.\left.e^{z_{2} L(-1)} \sigma_{12}\left(\mathcal{Y}_{a_{2} a_{3} ; j}^{\left.a_{5} ; 2\right)}\right)\left(w_{a_{3}}, e^{-\pi i} z_{2}\right) w_{a_{2}}\right\rangle\right) \\
& =e^{\pi i\left(h_{a_{1}}+h_{a_{5}}-h_{a_{2}}-h_{a_{3}}\right)} . \\
& \cdot E\left(\left\langle\sigma_{12}^{2}\left(\sigma_{23}\left(\mathcal{Y}_{a_{1} a_{5} ; i}^{a_{4} ;(1)}\right)\right)\left(e^{z_{1} L(1)}\left(e^{-\pi i} z_{1}^{-2}\right)^{L(0)} w_{a_{1}}, z_{1}^{-1}\right) w_{a_{4}^{\prime}},\right.\right. \\
& \left.e^{z_{2} L(-1)} \sigma_{23}^{2}\left(\sigma_{12}\left(\mathcal{Y}_{a_{2} a_{3} ; j}^{a_{5} ;(2)}\right)\left(w_{a_{3}}, e^{-\pi i} z_{2}\right) w_{a_{2}}\right\rangle\right) \\
& =e^{\pi i\left(2 h_{a_{5}}-h_{a_{2}}-h_{a_{4}}\right)} . \\
& \cdot E\left(\left\langle\sigma_{132}\left(\mathcal{Y}_{a_{2} a_{3} ; j}^{a_{5} ;(2)}\right)\left(e^{-z_{2} L(1)}\left(e^{-\pi i} z_{2}^{-2}\right)^{L(0)} w_{a_{3}}, e^{\pi i} z_{2}^{-1}\right) e^{z_{2} L(1)} e^{z_{1}^{-1} L(-1)} .\right.\right. \\
& \left.\left.\cdot \sigma_{123}\left(\mathcal{Y}_{a_{1} a_{5} ; i}^{a_{4} ;(1)}\right)\left(w_{a_{4}^{\prime}}, e^{-\pi i} z_{1}^{-1}\right) e^{z_{1} L(1)}\left(e^{-\pi i} z_{1}^{-2}\right)^{L(0)} w_{a_{1}}, w_{a_{2}}\right\rangle\right) .
\end{aligned}
$$

By (3.7), (3.8), the $L(-1)-, L(0)$ - and $L(1)$-conjugation formulas (see [FHL]), (1.1) and the associativity, the right-hand side of (4.5) is equal to

$$
\begin{gathered}
e^{\pi i\left(2 h_{a_{5}}-h_{a_{2}}-h_{a_{4}}\right)} \cdot \\
\cdot E\left(\left\langle\sigma_{132}\left(\mathcal{Y}_{a_{2} a_{3} ; j}^{\left.a_{5} ; 2\right)}\right)\left(e^{-z_{2} L(1)}\left(e^{-\pi i} z_{2}^{-2}\right)^{L(0)} w_{a_{3}}, e^{\pi i} z_{2}^{-1}\right) \cdot\right.\right. \\
\cdot e^{\left(z_{1}-z_{2}\right)^{-1} L(-1)} e^{z_{1}^{-1} z_{2}\left(z_{1}-z_{2}\right) L(1)}\left(z_{1}\left(z_{1}-z_{2}\right)^{-1}\right)^{2 L(0)} \\
\left.\left.\cdot \sigma_{123}\left(\mathcal{Y}_{a_{1} a_{5} ; i}^{a_{4} ;(1)}\right)\left(w_{a_{4}^{\prime}}, e^{-\pi i} z_{1}^{-1}\right) e^{z_{1} L(1)}\left(e^{-\pi i} z_{1}^{-2}\right)^{L(0)} w_{a_{1}}, w_{a_{2}}\right\rangle\right) \\
=e^{\pi i\left(2 h_{a_{5}}-h_{a_{2}}-h_{a_{4}}\right)} \cdot \\
\cdot E\left(\left\langlee ^ { ( z _ { 1 } - z _ { 2 } ) ^ { - 1 } L ( - 1 ) } \sigma _ { 1 3 2 } ( \mathcal { Y } _ { a _ { 2 } a _ { 3 } ; j } ^ { a _ { 5 } ; ( 2 ) } ) \left(e^{-z_{2} L(1)}\left(e^{-\pi i} z_{2}^{-2}\right)^{L(0)}\right.\right.\right. \\
\left.\cdot w_{a_{3}}, e^{\pi i} z_{1} z_{2}^{-1}\left(z_{1}-z_{2}\right)\right) e^{z_{1}^{-1} z_{2}\left(z_{1}-z_{2}\right) L(1)}
\end{gathered}
$$




$$
\begin{aligned}
& \cdot \sigma_{123}\left(\mathcal{Y}_{a_{1} a_{5} ; i}^{a_{4} ;(1)}\right)\left(\left(z_{1}\left(z_{1}-z_{2}\right)^{-1}\right)^{2 L(0)} w_{a_{4}^{\prime}}, e^{-\pi i} z_{1}^{-1}\left(z_{1}-z_{2}\right)^{-2}\right) \cdot \\
& \left.\left.\cdot\left(z_{1}\left(z_{1}-z_{2}\right)^{-1}\right)^{2 L(0)} e^{z_{1} L(1)}\left(e^{-\pi i} z_{1}^{-2}\right)^{L(0)} w_{a_{1}}, w_{a_{2}}\right\rangle\right) \\
& =e^{\pi i\left(2 h_{a_{5}}-h_{a_{2}}-h_{a_{4}}\right)} \text {. } \\
& \cdot E\left(\left\langlee ^ { ( z _ { 1 } - z _ { 2 } ) ^ { - 1 } L ( - 1 ) } \sigma _ { 1 3 2 } ( \mathcal { Y } _ { a _ { 2 } a _ { 3 } ; j } ^ { a _ { 5 } ( 2 ) } ) \left(e^{-z_{2} L(1)}\left(e^{-\pi i} z_{2}^{-2}\right)^{L(0)} \cdot\right.\right.\right. \\
& \left.\cdot w_{a_{3}}, e^{\pi i} z_{1} z_{2}^{-1}\left(z_{1}-z_{2}\right)\right) \sigma_{123}\left(\mathcal{Y}_{a_{1} a_{5} ; i}^{\left.a_{4} ; 1\right)}\right)\left(e^{z_{2} L(1)} w_{a_{4}^{\prime}}, e^{-\pi i}\left(z_{1}-z_{2}\right)^{-1}\right) \cdot \\
& \left.\left.\cdot e^{z_{1}^{-1} z_{2}\left(z_{1}-z_{2}\right) L(1)}\left(z_{1}\left(z_{1}-z_{2}\right)^{-1}\right)^{2 L(0)} e^{z_{1} L(1)}\left(e^{-\pi i} z_{1}^{-2}\right)^{L(0)} w_{a_{1}}, w_{a_{2}}\right\rangle\right) \\
& =e^{\pi i\left(2 h_{a_{5}}-h_{a_{2}}-h_{a_{4}}\right)} \text {. } \\
& \cdot E\left(\left\langlee ^ { ( z _ { 1 } - z _ { 2 } ) ^ { - 1 } L ( - 1 ) } \sigma _ { 1 3 2 } ( \mathcal { Y } _ { a _ { 2 } a _ { 3 } ; j } ^ { a _ { 5 } ; ( 2 ) } ) \left(e^{-z_{2} L(1)}\left(e^{-\pi i} z_{2}^{-2}\right)^{L(0)}\right.\right.\right. \text {. } \\
& \left.\cdot w_{a_{3}}, e^{2 \pi i} e^{-\pi i} z_{1} z_{2}^{-1}\left(z_{1}-z_{2}\right)\right) \cdot \\
& \cdot \sigma_{123}\left(\mathcal{Y}_{a_{1} a_{5} ; i}^{a_{4} ;(1)}\right)\left(e^{z_{2} L(1)} w_{a_{4}^{\prime}}, e^{-\pi i}\left(z_{1}-z_{2}\right)^{-1}\right) \cdot \\
& \left.\left.\cdot e^{\left(z_{1}-z_{2}\right) L(1)}\left(e^{-\pi i}\left(z_{1}-z_{2}\right)^{-2}\right)^{L(0)} w_{a_{1}}, w_{a_{2}}\right\rangle\right) \\
& =e^{\pi i\left(h_{a_{2}}-2 h_{a_{3}}-h_{a_{4}}\right)} \text {. } \\
& \cdot E\left(\left\langlee ^ { ( z _ { 1 } - z _ { 2 } ) ^ { - 1 } L ( - 1 ) } \sigma _ { 1 3 2 } ( \mathcal { Y } _ { a _ { 2 } a _ { 3 } ; j } ^ { a _ { 5 } ; ( 2 ) } ) \left(e^{-z_{2} L(1)}\left(e^{-\pi i} z_{2}^{-2}\right)^{L(0)}\right.\right.\right. \text {. } \\
& \left.\cdot w_{a_{3}}, e^{-\pi i} z_{1} z_{2}^{-1}\left(z_{1}-z_{2}\right)\right) \sigma_{123}\left(\mathcal{Y}_{a_{1} a_{5} ; i}^{a_{4} ;(1)}\right)\left(e^{z_{2} L(1)} w_{a_{4}^{\prime}}, e^{-\pi i}\left(z_{1}-z_{2}\right)^{-1}\right) \cdot \\
& \left.\left.\cdot e^{\left(z_{1}-z_{2}\right) L(1)}\left(e^{-\pi i}\left(z_{1}-z_{2}\right)^{-2}\right)^{L(0)} w_{a_{1}}, w_{a_{2}}\right\rangle\right) \\
& =e^{\pi i\left(h_{a_{2}}-2 h_{a_{3}}-h_{a_{4}}\right)} \sum_{a_{6} \in \mathcal{A}} \sum_{k=1}^{N_{a_{6} a_{3}}^{a_{4}}} \sum_{l=1}^{N_{a_{1} a_{2}}^{a_{6}}} \\
& F\left(\sigma_{132}\left(\mathcal{Y}_{a_{2} a_{3} ; j}^{\left.a_{5} ; 2\right)}\right) \otimes \sigma_{123}\left(\mathcal{Y}_{a_{1} a_{5} ; i}^{a_{4} ;(1)}\right) ; \sigma_{123}\left(\mathcal{Y}_{a_{1} a_{2} ; l}^{a_{6} ;(4)}\right) \otimes \sigma_{132}\left(\mathcal{Y}_{a_{6} a_{3} ; j}^{a_{4} ;(3)}\right)\right) \text {. } \\
& \cdot E\left(\left\langlee ^ { ( z _ { 1 } - z _ { 2 } ) ^ { - 1 } L ( - 1 ) } \sigma _ { 1 2 3 } ( \mathcal { Y } _ { a _ { 1 } a _ { 2 } ; j } ^ { a _ { 6 } ; ( 4 ) } ) \left(\sigma _ { 1 3 2 } ( \mathcal { Y } _ { a _ { 6 } a _ { 3 } ; i } ^ { a _ { 4 } ; 3 ) } ) \left(e^{-z_{2} L(1)}\left(e^{-\pi i} z_{2}^{-2}\right)^{L(0)}\right.\right.\right.\right. \text {. } \\
& \left.\left.\cdot w_{a_{3}}, e^{-\pi i} z_{2}^{-1}\right) e^{z_{2} L(1)} w_{a_{4}^{\prime}}, e^{-\pi i}\left(z_{1}-z_{2}\right)^{-1}\right) \cdot \\
& \left.\left.\cdot e^{\left(z_{1}-z_{2}\right) L(1)}\left(e^{-\pi i}\left(z_{1}-z_{2}\right)^{-2}\right)^{L(0)} w_{a_{1}}, w_{a_{2}}\right\rangle\right) \text {. }
\end{aligned}
$$

Using the definitions of $\sigma_{12}$ and $\sigma_{23}$, the relations $\sigma_{12}^{2}=\sigma_{23}^{2}=1, \sigma_{12} \sigma_{123}=$ $\sigma_{23}, \sigma_{23} \sigma_{132}=\sigma_{12}$, we see that the right-hand side of (3.14) is equal to

$$
\begin{aligned}
& e^{\pi i\left(h_{a_{6}}+h_{a_{1}}-2 h_{a_{3}}-h_{a_{4}}\right)} \sum_{a_{6} \in \mathcal{A}} \sum_{k=1}^{N_{a_{6} a_{3}}^{a_{4}}} \sum_{l=1}^{N_{a_{1} a_{2}}^{a_{6}}} \\
& F\left(\sigma_{132}\left(\mathcal{Y}_{a_{2} a_{3} ; j}^{a_{5} ;(2)}\right) \otimes \sigma_{123}\left(\mathcal{Y}_{a_{1} a_{5} ; i}^{a_{4} ;(1)}\right) ; \sigma_{123}\left(\mathcal{Y}_{a_{1} a_{2} ; l}^{a_{6} ;(4)}\right) \otimes \sigma_{132}\left(\mathcal{Y}_{a_{6} a_{3} ; j}^{a_{4} ;(3)}\right)\right) \cdot \\
& \quad \cdot E\left(\left\langle\sigma _ { 1 2 } ( \sigma _ { 1 2 3 } ( \mathcal { Y } _ { a _ { 1 } a _ { 2 } ; j } ^ { a _ { 6 } ; ( 4 ) } ) ) \left(e^{\left(z_{1}-z_{2}\right) L(1)}\left(e^{-\pi i}\left(z_{1}-z_{2}\right)^{-2}\right)^{L(0)} .\right.\right.\right. \\
& \left.\quad \cdot w_{a_{1}},\left(z_{1}-z_{2}\right)^{-1}\right) \sigma_{132}\left(\mathcal{Y}_{a_{6} a_{3} ; i}^{a_{4} ;(3)}\right)\left(e^{-z_{2} L(1)}\left(e^{-\pi i} z_{2}^{-2}\right)^{L(0)} w_{a_{3}}, e^{-2 \pi i} e^{\pi i} z_{2}^{-1}\right) .
\end{aligned}
$$




$$
\left.\left.\cdot e^{z_{2} L(1)} w_{a_{4}^{\prime}}, w_{a_{2}}\right\rangle\right)
$$

$$
\begin{aligned}
& =e^{\pi i\left(h_{a_{1}}+h_{a_{4}}-h_{a_{6}}\right)} \sum_{a_{6} \in \mathcal{A}} \sum_{k=1}^{N_{a_{6} a_{3}}^{a_{4}}} \sum_{l=1}^{N_{a_{1} a_{2}}^{a_{6}}} \\
& F\left(\sigma_{132}\left(\mathcal{Y}_{a_{2} a_{3} ; j}^{\left.a_{5} ; 2\right)}\right) \otimes \sigma_{123}\left(\mathcal{Y}_{a_{1} a_{5} ; i}^{a_{4} ;(1)}\right) ; \sigma_{123}\left(\mathcal{Y}_{a_{1} a_{2} ; l}^{\left.a_{6} ; 4\right)}\right) \otimes \sigma_{132}\left(\mathcal{Y}_{a_{6} a_{3} ; j}^{a_{4} ;(3)}\right)\right) . \\
& \cdot E\left(\left\langle\sigma_{23}\left(\mathcal{Y}_{a_{1} a_{2} ; j}^{\left.a_{6} ; 4\right)}\right)\left(e^{\left(z_{1}-z_{2}\right) L(1)}\left(e^{-\pi i}\left(z_{1}-z_{2}\right)^{-2}\right)^{L(0)} w_{a_{1}},\left(z_{1}-z_{2}\right)^{-1}\right) \cdot\right.\right. \\
& \left.\cdot \sigma_{132}\left(\mathcal{Y}_{a_{6} a_{3} ; i}^{a_{4} ;(3)}\left(e^{-z_{2} L(1)}\left(e^{-\pi i} z_{2}^{-2}\right)^{L(0)} w_{a_{3}}, e^{\pi i} z_{2}^{-1}\right) e^{z_{2} L(1)} w_{a_{4}^{\prime}}, w_{a_{2}}\right\rangle\right) \\
& =e^{\pi i\left(h_{a_{4}}-h_{a_{3}}-h_{a_{6}}\right)} \sum_{a_{6} \in \mathcal{A}} \sum_{k=1}^{N_{a_{6} a_{3}}^{a_{4}}} \sum_{l=1}^{N_{a_{1} a_{2}}^{a_{6}}} \\
& F\left(\sigma_{132}\left(\mathcal{Y}_{a_{2} a_{3} ; j}^{a_{5}(2)}\right) \otimes \sigma_{123}\left(\mathcal{Y}_{a_{1} a_{5} ; i}^{a_{4} ;(1)}\right) ; \sigma_{123}\left(\mathcal{Y}_{a_{1} a_{2} ; l}^{\left.a_{6} ; 4\right)}\right) \otimes \sigma_{132}\left(\mathcal{Y}_{a_{6} a_{3} ; j}^{a_{4} ;(3)}\right)\right) \cdot \\
& \cdot E\left(\left\langlew_{a_{4}^{\prime}}, e^{z_{2} L(-1)} \sigma_{23}\left(\sigma_{132}\left(\mathcal{Y}_{a_{6} a_{3} ; i}^{\left.a_{4} ; 3\right)}\right)\left(w_{a_{3}}, e^{-\pi i} z_{2}\right) \cdot\right.\right.\right. \\
& \left.\left.\cdot \sigma_{23}^{2}\left(\mathcal{Y}_{a_{1} a_{2} ; j}^{\left.a_{6} ; 4\right)}\right)\left(w_{a_{1}}, z_{1}-z_{2}\right) w_{a_{2}}\right\rangle\right) \\
& =e^{\pi i\left(h_{a_{4}}-h_{a_{3}}-h_{a_{6}}\right)} \sum_{a_{6} \in \mathcal{A}} \sum_{k=1}^{N_{a_{6} a_{3}}^{a_{4}}} \sum_{l=1}^{N_{a_{1} a_{2}}^{a_{6}}} \\
& F\left(\sigma_{132}\left(\mathcal{Y}_{a_{2} a_{3} ; j}^{a_{5}(2)}\right) \otimes \sigma_{123}\left(\mathcal{Y}_{a_{1} a_{5} ; i}^{a_{4} ;(1)}\right) ; \sigma_{123}\left(\mathcal{Y}_{a_{1} a_{2} ; l}^{a_{6} ;(4)}\right) \otimes \sigma_{132}\left(\mathcal{Y}_{a_{6} a_{3} ; j}^{a_{4} ;(3)}\right)\right) . \\
& \cdot E\left(\left\langlew_{a_{4}^{\prime}}, e^{z_{2} L(-1)} \sigma_{12}\left(\mathcal{Y}_{a_{6} a_{3} ; i}^{a_{4} ;(3)}\right)\left(w_{a_{3}}, e^{-\pi i} z_{2}\right)\right.\right. \text {. } \\
& \left.\left.\cdot \sigma_{23}^{2}\left(\mathcal{Y}_{a_{1} a_{2} ; j}^{a_{6} ;(4)}\right)\left(w_{a_{1}}, z_{1}-z_{2}\right) w_{a_{2}}\right\rangle\right) \\
& =\sum_{a_{6} \in \mathcal{A}} \sum_{k=1}^{N_{a_{6} a_{3}}^{a_{4}}} \sum_{l=1}^{N_{a_{1} a_{2}}^{a_{6}}} \\
& F\left(\sigma_{132}\left(\mathcal{Y}_{a_{2} a_{3} ; j}^{a_{5} ;(2)}\right) \otimes \sigma_{123}\left(\mathcal{Y}_{a_{1} a_{5} ; i}^{a_{4} ; 1}\right) ; \sigma_{123}\left(\mathcal{Y}_{a_{1} a_{2} ; l}^{a_{6} ;(4)}\right) \otimes \sigma_{132}\left(\mathcal{Y}_{a_{6} a_{3} ; j}^{a_{4} ;(3)}\right)\right) . \\
& \cdot E\left(\left\langle w_{a_{4}^{\prime}}, \sigma_{12}^{2}\left(\mathcal{Y}_{a_{6} a_{3} ; i}^{\left.a_{4} ; 3\right)}\right)\left(\sigma_{23}^{2}\left(\mathcal{Y}_{a_{1} a_{2} ; j}^{a_{6} ;(4)}\right)\left(w_{a_{1}}, z_{1}-z_{2}\right) w_{a_{2}}, z_{2}\right) w_{a_{3}}\right\rangle\right) \\
& =\sum_{a_{6} \in \mathcal{A}} \sum_{k=1}^{N_{a_{6} a_{3}}^{a_{4}}} \sum_{l=1}^{N_{a_{1} a_{2}}^{a_{6}}} \\
& F\left(\sigma_{132}\left(\mathcal{Y}_{a_{2} a_{3} ; j}^{a_{5} ;(2)}\right) \otimes \sigma_{123}\left(\mathcal{Y}_{a_{1} a_{5} ; i}^{a_{4} ; 1}\right) ; \sigma_{123}\left(\mathcal{Y}_{a_{1} a_{2} ; l}^{a_{6} ;(4)}\right) \otimes \sigma_{132}\left(\mathcal{Y}_{a_{6} a_{3} ; j}^{a_{4} ;(3)}\right)\right) . \\
& \cdot E\left(\left\langle w_{a_{4}^{\prime}}, \mathcal{Y}_{a_{6} a_{3} ; i}^{a_{4} ;(3)}\left(\mathcal{Y}_{a_{1} a_{2} ; j}^{a_{6} ;(4)}\left(w_{a_{1}}, z_{1}-z_{2}\right) w_{a_{2}}, z_{2}\right) w_{a_{3}}\right\rangle\right) .
\end{aligned}
$$

Using (3.13) -(3.15), we see that the left-hand side of (3.13) is equal to the right-hand side of (3.15). Comparing this resulting equality with (1.5) 
and using Proposition 1.2, we obtain (3.12).

\section{Moore-Seiberg formulas}

In [MS1], Moore and Seiberg derived two formulas from the (assumed) axioms for rational conformal field theories. The Verlinde conjecture is a consequence of these equations. In this section, we prove these formulas mathematically using the results obtained in the representation theory of vertex operator algebras, especially those obtained in [H6] and [H7] and in the preceding sections.

We now want to choose a basis $\mathcal{Y}_{a_{1} a_{2} ; i}^{a_{3}}, i=1, \ldots, N_{a_{1} a_{2}}^{a_{3}}$, of $\mathcal{V}_{a_{1} a_{2}}^{a_{3}}$ for each triple $a_{1}, a_{2}, a_{3} \in \mathcal{A}$. Note that for each element $\sigma \in S_{3}, \sigma(\mathcal{Y})_{a_{1} a_{2} ; i}^{a_{3}}$, $i=1, \ldots, N_{a_{1} a_{2}}^{a_{3}}$, is also a basis of $\mathcal{V}_{a_{1} a_{2}}^{a_{3}}$.

For $a \in \mathcal{A}$, we choose $\mathcal{Y}_{e a ; 1}^{a}$ to be the the vertex operator $Y_{W^{a}}$ defining the module structure on $W^{a}$ and we choose $\mathcal{Y}_{a e ; 1}^{a}$ to be the intertwining operator defined using the action of $\sigma_{12}$, or equivalently the skew-symmetry in this case,

$$
\begin{aligned}
\mathcal{Y}_{a e ; 1}^{a}\left(w_{a}, x\right) u & =\sigma_{12}\left(\mathcal{Y}_{e a ; 1}^{a}\right)\left(w_{a}, x\right) u \\
& =e^{x L(-1)} \mathcal{Y}_{e a ; 1}^{a}(u,-x) w_{a} \\
& =e^{x L(-1)} Y_{W^{a}}(u,-x) w_{a}
\end{aligned}
$$

for $u \in V$ and $w_{a} \in W^{a}$. Since $V^{\prime}$ as a $V$-module is isomorphic to $V$, we have $e^{\prime}=e$. From [FHL], we know that there is a nondegerate invariant bilinear form $(\cdot, \cdot)$ on $V$ such that $(\mathbf{1}, \mathbf{1})=1$. We choose $\mathcal{Y}_{a a^{\prime} ; 1}^{e}=\mathcal{Y}_{a a^{\prime} ; 1}^{e^{\prime}}$ to be the intertwining operator defined using the action of $\sigma_{23}$ by

$$
\mathcal{Y}_{a a^{\prime} ; 1}^{e^{\prime}}=\sigma_{23}\left(\mathcal{Y}_{a e ; 1}^{a}\right)
$$

that is,

$$
\left(u, \mathcal{Y}_{a a^{\prime} ; 1}^{e^{\prime}}\left(w_{a}, x\right) w_{a^{\prime}}\right)=e^{\pi i h_{a}}\left\langle\mathcal{Y}_{a e ; 1}^{a}\left(e^{x L(1)}\left(e^{-\pi i} x^{-2}\right)^{L(0)} w_{a}, x^{-1}\right) u, w_{a^{\prime}}\right\rangle
$$

for $u \in V, w_{a} \in W^{a}$ and $w_{a^{\prime}} \in W^{a^{\prime}}$. Since the actions of $\sigma_{12}$ and $\sigma_{23}$ generate the action of $S_{3}$ on $\mathcal{V}$, we have

$$
\mathcal{Y}_{a^{\prime} a ; 1}^{e}=\sigma_{12}\left(\mathcal{Y}_{a a^{\prime} ; 1}^{e}\right)
$$


for any $a \in \mathcal{A}$. When $a_{1}, a_{2}, a_{3} \neq e$, we choose $\mathcal{Y}_{a_{1} a_{2} ; i}^{a_{3}}, i=1, \ldots, N_{a_{1} a_{2}}^{a_{3}}$, to be an arbitrary basis of $\mathcal{V}_{a_{1} a_{2}}^{a_{3}}$.

Recall that $\sigma_{123}=\sigma_{12} \sigma_{23}$ and $\sigma_{132}=\sigma_{23} \sigma_{12}$. The following theorem gives the first Moore-Seiberg formula in [MS1]:

Theorem 4.1 For $a_{1}, a_{2}, a_{3} \in \mathcal{A}$, we have

$$
\begin{gathered}
\sum_{i=1}^{N_{a_{1} a_{2}}^{a_{3}}} \sum_{k=1}^{N_{a_{1}^{\prime} a_{3}}^{a_{2}}} F\left(\mathcal{Y}_{a_{2} e ; 1}^{a_{2}} \otimes \mathcal{Y}_{a_{3}^{\prime} a_{3} ; 1}^{e} ; \mathcal{Y}_{a_{1}^{\prime} a_{3} ; k}^{a_{2}} \otimes \mathcal{Y}_{a_{2} a_{3}^{\prime} ; i}^{a_{1}^{\prime}}\right) . \\
\cdot F\left(\mathcal{Y}_{a_{1}^{\prime} a_{3} ; k}^{a_{2}} \otimes \sigma_{123}\left(\mathcal{Y}_{a_{2} a_{3}^{\prime} ;}^{a_{1}^{\prime}}\right) ; \mathcal{Y}_{e a_{2} ; 1}^{a_{2}} \otimes \mathcal{Y}_{a_{1}^{\prime} a_{1} ; 1}^{e}\right) \\
=N_{a_{1} a_{2}}^{a_{3}} F\left(\mathcal{Y}_{a_{2} e ; 1}^{a_{2}} \otimes \mathcal{Y}_{a_{2}^{\prime} a_{2} ; 1}^{e} ; \mathcal{Y}_{e a_{2} ; 1}^{a_{2}} \otimes \mathcal{Y}_{a_{2} a_{2}^{\prime} ; 1}^{e}\right) .
\end{gathered}
$$

Proof. For $a_{1}, a_{2}, a_{3} \in \mathcal{A}, w_{a_{1}} \in W^{a_{1}}, w_{a_{3}^{\prime}} \in W^{a_{3}^{\prime}}, w_{a_{2}}^{1}, w_{a_{2}}^{2} \in W^{a_{2}}, w_{a_{2}^{\prime}} \in$ $W^{a_{2}^{\prime}}, i=1, \ldots, N_{a_{1} a_{2}}^{a_{3}}$, by the associativity (1.5), we have

$$
\begin{aligned}
& E\left(\left\langle w_{a_{2}^{\prime}}, \mathcal{Y}_{a_{2} e ; 1}^{a_{2}}\left(w_{a_{2}}^{1}, z_{1}\right) \mathcal{Y}_{a_{3}^{\prime} a_{3} ; 1}^{e}\left(w_{a_{3}^{\prime}}, z_{2}\right) \sigma_{123}\left(\mathcal{Y}_{a_{2} a_{3}^{\prime} ; i}^{a_{1}^{\prime}}\right)\left(w_{a_{1}}, z_{3}\right) w_{a_{2}}^{2}\right\rangle\right) \\
& =\sum_{a_{4} \in \mathcal{A}} \sum_{j=1}^{N_{a_{2} a_{3}^{\prime}}^{a_{4}}} \sum_{k=1}^{N_{a_{4} a_{3}}^{a_{2}}} F\left(\mathcal{Y}_{a_{2} e ; 1}^{a_{2}} \otimes \mathcal{Y}_{a_{3}^{\prime} a_{3} ; 1}^{e} ; \mathcal{Y}_{a_{4} a_{3} ; k}^{a_{2}} \otimes \mathcal{Y}_{a_{2} a_{3}^{\prime} ; j}^{a_{4}}\right) \text {. } \\
& \cdot E\left(\left\langle w_{a_{2}^{\prime}}, \mathcal{Y}_{a_{4} a_{3} ; k}^{a_{2}}\left(\mathcal{Y}_{a_{2} a_{3}^{\prime} ; j}^{a_{4}}\left(w_{a_{2}}^{1}, z_{1}-z_{2}\right) w_{a_{3}^{\prime}}, z_{2}\right) \sigma_{123}\left(\mathcal{Y}_{a_{2} a_{3}^{\prime} ;}^{a_{1}^{\prime}}\right)\left(w_{a_{1}}, z_{3}\right) w_{a_{2}}^{2}\right\rangle\right)
\end{aligned}
$$

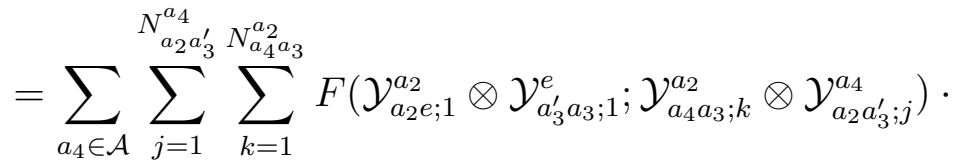

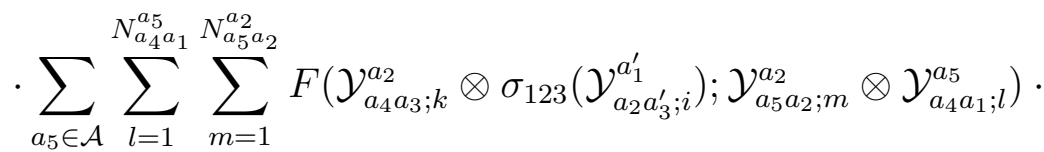

$$
\begin{aligned}
& \cdot E\left(\left\langle w_{a_{2}^{\prime}}, \mathcal{Y}_{a_{5} a_{2} ; m}^{a_{2}}\left(\mathcal{Y}_{a_{4} a_{1} ; l}^{a_{5}}\left(\mathcal{Y}_{a_{2} a_{3}^{\prime} ; j}^{a_{4}}\left(w_{a_{2}}^{1}, z_{1}-z_{2}\right) w_{a_{3}^{\prime}}, z_{2}-z_{3}\right) w_{a_{1}}, z_{3}\right) w_{a_{2}}^{2}\right\rangle\right)
\end{aligned}
$$

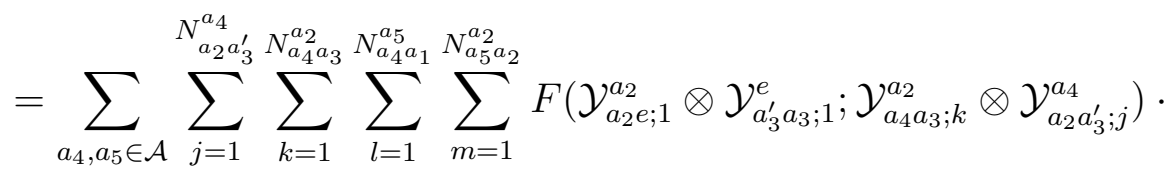

$$
\begin{aligned}
& \cdot F\left(\mathcal{Y}_{a_{4} a_{3} ; k}^{a_{2}} \otimes \sigma_{123}\left(\mathcal{Y}_{a_{2} a_{3}^{\prime} ;}^{a_{1}^{\prime}}\right) ; \mathcal{Y}_{a_{5} a_{2} ; m}^{a_{2}} \otimes \mathcal{Y}_{a_{4} a_{1} ; l}^{a_{5}}\right) \cdot \\
& \cdot E\left(\left\langle w_{a_{2}^{\prime}}, \mathcal{Y}_{a_{5} a_{2} ; m}^{a_{2}}\left(\mathcal{Y}_{a_{4} a_{1} ; l}^{a_{5}}\left(\mathcal{Y}_{a_{2} a_{3}^{\prime} ; j}^{a_{4}}\left(w_{a_{2}}^{1}, z_{1}-z_{2}\right) w_{a_{3}^{\prime}}, z_{2}-z_{3}\right) w_{a_{1}}, z_{3}\right) w_{a_{2}}^{2}\right\rangle\right) \text {. }
\end{aligned}
$$


On the other hand, also by the associativity (1.5), we have

$$
\begin{aligned}
& E\left(\left\langle w_{a_{2}^{\prime}}, \mathcal{Y}_{a_{2} e ; 1}^{a_{2}}\left(w_{a_{2}}^{1}, z_{1}\right) \mathcal{Y}_{a_{3}^{\prime} a_{3} ; 1}^{e}\left(w_{a_{3}^{\prime}}, z_{2}\right) \sigma_{123}\left(\mathcal{Y}_{a_{2} a_{3}^{\prime} ; i}^{a_{1}^{\prime}}\right)\left(w_{a_{1}}, z_{3}\right) w_{a_{2}}^{2}\right\rangle\right)
\end{aligned}
$$

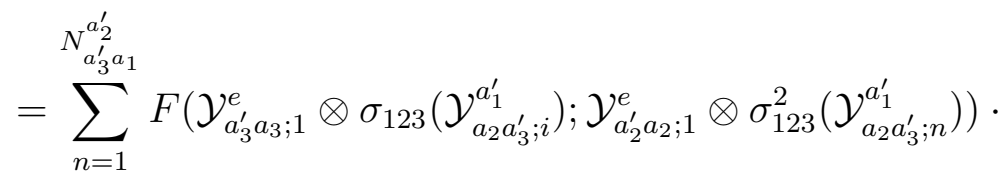

$$
\begin{aligned}
& \cdot E\left(\left\langle w_{a_{2}^{\prime}}, \mathcal{Y}_{a_{2} e ; 1}^{a_{2}}\left(w_{a_{2}}^{1}, z_{1}\right) \mathcal{Y}_{a_{2}^{\prime} a_{2} ; 1}^{e}\left(\sigma_{123}^{2}\left(\mathcal{Y}_{a_{2} a_{3}^{\prime} ; n}^{a_{1}^{\prime}}\right)\left(w_{a_{3}^{\prime}}, z_{2}-z_{3}\right) w_{a_{1}}, z_{3}\right) w_{a_{2}}^{2}\right\rangle\right)
\end{aligned}
$$

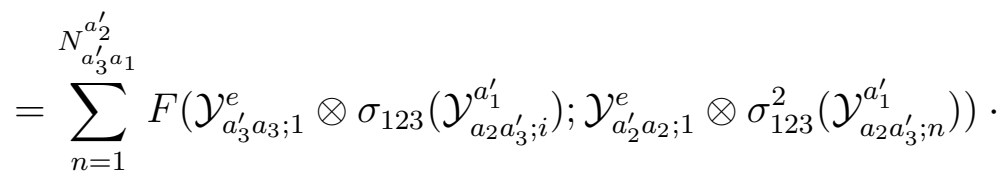

$$
\begin{aligned}
& \cdot \sum_{a_{6} \in \mathcal{A}} \sum_{r=1}^{N_{a_{2} a_{2}^{\prime}}^{a_{6}}} \sum_{s=1}^{N_{a_{6} a_{2}}^{a_{2}}} F\left(\mathcal{Y}_{a_{2} e ; 1}^{a_{2}} \otimes \mathcal{Y}_{a_{2}^{\prime} a_{2} ; 1}^{e} ; \mathcal{Y}_{a_{6} a_{2} ; s}^{a_{2}} \otimes \mathcal{Y}_{a_{2} a_{2}^{\prime} ; r}^{a_{6}}\right) . \\
& \cdot E\left(\left\langlew_{a_{2}^{\prime}}, \mathcal{Y}_{a_{6} a_{2} ; s}^{a_{2}}\left(\mathcal{Y}_{a_{2} a_{2}^{\prime} ; r}^{a_{6}}\left(w_{a_{2}}^{1}, z_{1}-z_{3}\right) \cdot\right.\right.\right. \\
& \left.\left.\left.\cdot \sigma_{123}^{2}\left(\mathcal{Y}_{a_{2} a_{3}^{\prime} ; n}^{a_{1}^{\prime}}\right)\left(w_{a_{3}^{\prime}}, z_{2}-z_{3}\right) w_{a_{1}}, z_{3}\right) w_{a_{2}}^{2}\right\rangle\right)
\end{aligned}
$$

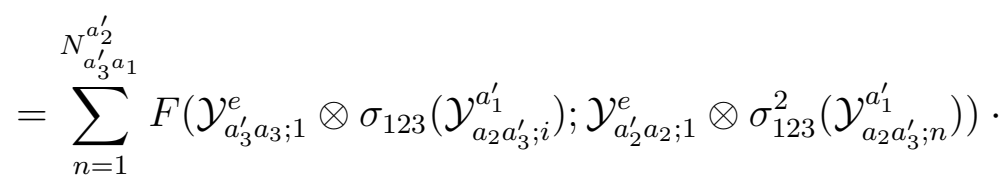

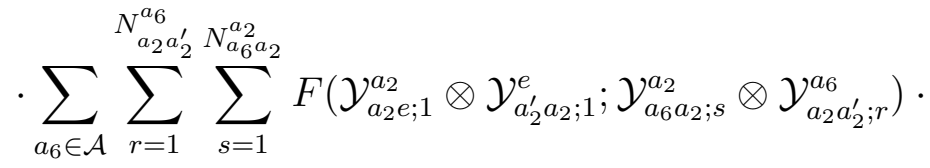

$$
\begin{aligned}
& \cdot \sum_{a_{7} \in \mathcal{A}} \sum_{p=1}^{N_{a_{2} a_{3}^{\prime}}^{a_{7}}} \sum_{q=1}^{N_{a_{7} a_{1}}^{a_{a}}} F\left(\mathcal{Y}_{a_{2} a_{2}^{\prime} ; r}^{a_{6}} \otimes \sigma_{123}^{2}\left(\mathcal{Y}_{a_{2} a_{3}^{\prime} ; n}^{a_{1}^{\prime}}\right) ; \mathcal{Y}_{a_{7} a_{1} ; q}^{a_{a_{6}}} \otimes \mathcal{Y}_{a_{2} a_{3}^{\prime} ; p}^{a_{7}}\right) \text {. } \\
& \cdot E\left(\left\langle w_{a_{2}^{\prime}}, \mathcal{Y}_{a_{6} a_{2} ; s}^{a_{2}}\left(\mathcal{Y}_{a_{7} a_{1} ; q}^{a_{6}}\left(\mathcal{Y}_{a_{2} a_{3}^{\prime} ; p}^{a_{7}}\left(w_{a_{2}}^{1}, z_{1}-z_{2}\right) w_{a_{3}^{\prime}}, z_{2}-z_{3}\right) w_{a_{1}}, z_{3}\right) w_{a_{2}}^{2}\right\rangle\right) \\
& =\sum_{a_{6}, a_{7} \in \mathcal{A}} \sum_{n=1}^{N_{a_{3}^{\prime} a_{1}}^{a^{\prime}}} \sum_{r=1}^{N_{a_{2} a_{2}^{\prime}}^{a_{6}}} \sum_{s=1}^{N_{a_{6} a_{2}}^{a_{2}}} \sum_{p=1}^{N_{a_{2} a_{3}^{\prime}}^{a_{7}}} \sum_{q=1}^{N_{a_{7} a_{1}}^{a_{6}}} \\
& F\left(\mathcal{Y}_{a_{3}^{\prime} a_{3} ; 1}^{e} \otimes \sigma_{123}\left(\mathcal{Y}_{a_{2} a_{3}^{\prime} ; i}^{a_{1}^{\prime}}\right) ; \mathcal{Y}_{a_{2}^{\prime} a_{2} ; 1}^{e} \otimes \sigma_{123}^{2}\left(\mathcal{Y}_{a_{2} a_{3}^{\prime} ; n}^{a_{1}^{\prime}}\right)\right) \\
& \cdot F\left(\mathcal{Y}_{a_{2} e ; 1}^{a_{2}} \otimes \mathcal{Y}_{a_{2}^{\prime} a_{2} ; 1}^{e} ; \mathcal{Y}_{a_{6} a_{2} ; s}^{a_{2}} \otimes \mathcal{Y}_{a_{2} a_{2}^{\prime} ; r}^{a_{6}}\right) \text {. } \\
& \cdot F\left(\mathcal{Y}_{a_{2} a_{2}^{\prime} ; r}^{a_{6}} \otimes \sigma_{123}^{2}\left(\mathcal{Y}_{a_{2} a_{3}^{\prime} ; n}^{a_{1}^{\prime}}\right) ; \mathcal{Y}_{a_{7} a_{1} ; q}^{a_{6}} \otimes \mathcal{Y}_{a_{2} a_{3}^{\prime} ; p}^{a_{7}}\right) \cdot
\end{aligned}
$$




$$
\cdot E\left(\left\langle w_{a_{2}^{\prime}}, \mathcal{Y}_{a_{6} a_{2} ; s}^{a_{2}}\left(\mathcal{Y}_{a_{7} a_{1} ; q}^{a_{6}}\left(\mathcal{Y}_{a_{2} a_{3}^{\prime} ; p}^{a_{7}}\left(w_{a_{2}}^{1}, z_{1}-z_{2}\right) w_{a_{3}^{\prime}}, z_{2}-z_{3}\right) w_{a_{1}}, z_{3}\right) w_{a_{2}}^{2}\right\rangle\right)
$$

Using (4.2)-(4.3) and Proposition 1.3, we obtain

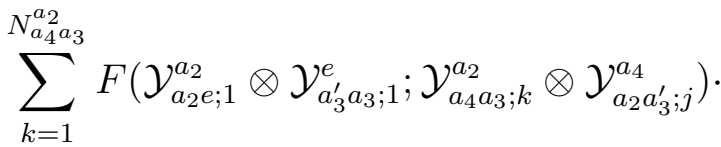

$$
\begin{aligned}
& \cdot F\left(\mathcal{Y}_{a_{4} a_{3} ; k}^{a_{2}} \otimes \sigma_{123}\left(\mathcal{Y}_{a_{2} a_{3}^{\prime} ; i}^{a_{1}^{\prime}}\right) ; \mathcal{Y}_{a_{5} a_{2} ; m}^{a_{2}} \otimes \mathcal{Y}_{a_{4} a_{1} ; l}^{a_{5}}\right) \\
& =\sum_{n=1}^{N_{a_{3}^{\prime} a_{1}}^{a^{\prime}}} \sum_{r=1}^{N_{a_{2} a_{2}^{\prime}}^{a_{2}}} F\left(\mathcal{Y}_{a_{3}^{\prime} a_{3} ; 1}^{e} \otimes \sigma_{123}\left(\mathcal{Y}_{a_{2} a_{3}^{\prime} ;}^{a_{1}^{\prime}}\right) ; \mathcal{Y}_{a_{2}^{\prime} a_{2} ; 1}^{e} \otimes \sigma_{123}^{2}\left(\mathcal{Y}_{a_{2} a_{3}^{\prime} ; n}^{a_{1}^{\prime}}\right)\right) \text {. } \\
& \cdot F\left(\mathcal{Y}_{a_{2} e ; 1}^{a_{2}} \otimes \mathcal{Y}_{a_{2}^{\prime} a_{2} ; 1}^{e} ; \mathcal{Y}_{a_{5} a_{2} ; m}^{a_{2}} \otimes \mathcal{Y}_{a_{2} a_{2}^{\prime} ; r}^{a_{5}}\right) \cdot \\
& \cdot F\left(\mathcal{Y}_{a_{2} a_{2}^{\prime} ; r}^{a_{5}} \otimes \sigma_{123}^{2}\left(\mathcal{Y}_{a_{2} a_{3}^{\prime} ; n}^{a_{1}^{\prime}}\right) ; \mathcal{Y}_{a_{4} a_{1} ; l}^{a_{5}} \otimes \mathcal{Y}_{a_{2} a_{3}^{\prime} ; j}^{a_{4}}\right) \text {. }
\end{aligned}
$$

In particular, for $a_{5}=e$ and $a_{4}=a_{1}^{\prime}$, we have

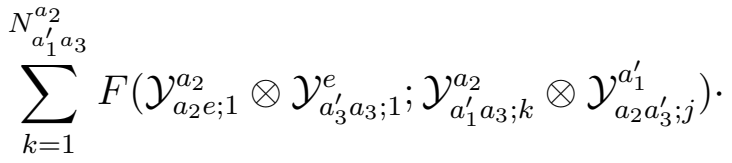

$$
\begin{aligned}
& \cdot F\left(\mathcal{Y}_{a_{4} a_{3} ; k}^{a_{2}} \otimes \sigma_{123}\left(\mathcal{Y}_{a_{2} a_{3}^{\prime} ;}^{a_{1}^{\prime}}\right) ; \mathcal{Y}_{e a_{2} ; m}^{a_{2}} \otimes \mathcal{Y}_{a_{1}^{\prime} a_{1} ; l}^{e}\right) \\
& =\sum_{n=1}^{N_{a_{3}^{\prime} a_{1}}^{a_{2}^{\prime}}} F\left(\mathcal{Y}_{a_{3}^{\prime} a_{3} ; 1}^{e} \otimes \sigma_{123}\left(\mathcal{Y}_{a_{2} a_{3}^{\prime} ; i}^{a_{1}^{\prime}}\right) ; \mathcal{Y}_{a_{2}^{\prime} a_{2} ; 1}^{e} \otimes \sigma_{123}^{2}\left(\mathcal{Y}_{a_{2} a_{3}^{\prime} ; n}^{a_{1}^{\prime}}\right)\right) \text {. } \\
& \cdot F\left(\mathcal{Y}_{a_{2} e ; 1}^{a_{2}} \otimes \mathcal{Y}_{a_{2}^{\prime} a_{2} ; 1}^{e} ; \mathcal{Y}_{e a_{2} ; 1}^{a_{2}} \otimes \mathcal{Y}_{a_{2} a_{2}^{\prime} ; 1}^{e}\right) \text {. } \\
& \cdot F\left(\mathcal{Y}_{a_{2} a_{2}^{\prime} ; 1}^{e} \otimes \sigma_{123}^{2}\left(\mathcal{Y}_{a_{2} a_{3}^{\prime} ; n}^{a_{1}^{\prime}}\right) ; \mathcal{Y}_{a_{1}^{\prime} a_{1} ; 1}^{e} \otimes \mathcal{Y}_{a_{2} a_{3}^{\prime} ; j}^{a^{\prime}}\right)
\end{aligned}
$$

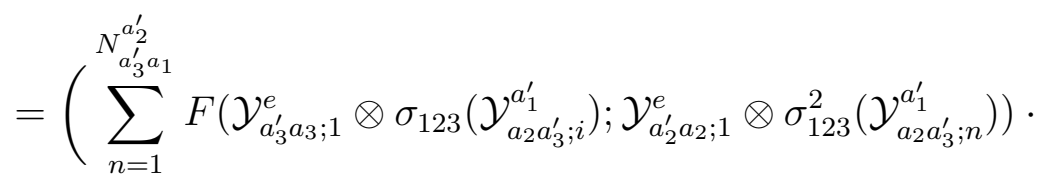

$$
\begin{aligned}
& \left.\cdot F\left(\mathcal{Y}_{a_{2} a_{2}^{\prime} ; 1}^{e} \otimes \sigma_{123}^{2}\left(\mathcal{Y}_{a_{2} a_{3}^{\prime} ; n}^{a_{1}^{\prime}}\right) ; \mathcal{Y}_{a_{1}^{\prime} a_{1} ; 1}^{e} \otimes \mathcal{Y}_{a_{2} a_{3}^{\prime} ; j}^{a_{1}^{\prime}}\right)\right) \\
& \cdot F\left(\mathcal{Y}_{a_{2} e ; 1}^{a_{2}} \otimes \mathcal{Y}_{a_{2}^{\prime} a_{2} ; 1}^{e} ; \mathcal{Y}_{e a_{2} ; 1}^{a_{2}} \otimes \mathcal{Y}_{a_{2} a_{2}^{\prime} ; 1}^{e}\right) \text {. }
\end{aligned}
$$

On the other hand, by the definition of $\sigma_{12}$ and $\sigma_{23}$, the relations $\sigma_{12}^{2}=$ $\sigma_{23}^{2}=1$ and the choices of the bases $\mathcal{Y}_{e a ; 1}^{a}, \mathcal{Y}_{a e ; 1}^{a}, \mathcal{Y}_{a a^{\prime}}^{e}$ and $\mathcal{Y}_{a^{\prime} a ; 1}^{e}$ for $a \in \mathcal{A}$, 
we have

$$
\begin{aligned}
& \left\langle u, \mathcal{Y}_{a_{3}^{\prime} a_{3} ; 1}^{e}\left(w_{a_{3}^{\prime}}, x_{1}\right) \sigma_{123}\left(\mathcal{Y}_{a_{2} a_{3}^{\prime} ;}^{a_{1}^{\prime}}\right)\left(w_{a_{1}}, x_{2}\right) w_{a_{2}}\right\rangle \\
& =\left\langle u, \sigma_{23}^{2}\left(\mathcal{Y}_{a_{3}^{\prime} a_{3} ; 1}^{e}\right)\left(w_{a_{3}^{\prime}}, x_{1}\right) \sigma_{23}^{2}\left(\sigma_{123}\left(\mathcal{Y}_{a_{2} a_{3}^{\prime} ;}^{a_{1}^{\prime}}\right)\right)\left(w_{a_{1}}, x_{2}\right) w_{a_{2}}\right\rangle \\
& =e^{\pi i\left(h_{a_{1}}+h_{a_{3}}\right)}\left\langle\sigma_{23}\left(\sigma_{123}\left(\mathcal{Y}_{a_{2} a_{3}^{\prime} ; i}^{a_{1}^{\prime}}\right)\right)\left(e^{x_{2} L(1)}\left(e^{-\pi i} x_{2}^{-2}\right)^{L(0)} w_{a_{1}}, x_{2}^{-1}\right)\right. \text {. } \\
& \left.\cdot \sigma_{23}\left(\mathcal{Y}_{a_{3}^{\prime} a_{3} ; 1}^{e}\right)\left(e^{x_{1} L(1)}\left(e^{-\pi i} x_{1}^{-2}\right)^{L(0)} w_{a_{3}^{\prime}}, x_{1}^{-1}\right) u, w_{a_{2}}\right\rangle \\
& =e^{\pi i\left(h_{a_{1}}+h_{a_{3}}\right)}\left\langle\sigma_{23}\left(\sigma_{123}\left(\mathcal{Y}_{a_{2} a_{3}^{\prime} ;}^{a_{1}^{\prime}}\right)\right)\left(e^{x_{2} L(1)}\left(e^{-\pi i} x_{2}^{-2}\right)^{L(0)} w_{a_{1}}, x_{2}^{-1}\right)\right. \text {. } \\
& \text { - } \left.\mathcal{Y}_{a_{3}^{\prime} e ; 1}^{a_{3}^{\prime}}\left(e^{x_{1} L(1)}\left(e^{-\pi i} x_{1}^{-2}\right)^{L(0)} w_{a_{3}^{\prime}}, x_{1}^{-1}\right) u, w_{a_{2}}\right\rangle \\
& =e^{\pi i\left(h_{a_{1}}+h_{a_{3}}\right)}\left\langle\sigma _ { 1 2 } ( \sigma _ { 1 2 3 } ( \mathcal { Y } _ { a _ { 2 } a _ { 3 } ^ { \prime } ; i } ^ { a _ { 1 } ^ { \prime } } ) ) \left( e^{x_{2} L(1)}\left(\left(e^{-\pi i} x_{2}^{-2}\right)^{L(0)} w_{a_{1}}, x_{2}^{-1}\right)\right.\right. \text {. } \\
& \cdot e^{x_{1}^{-1} L(-1)} \mathcal{Y}_{e a_{3}^{\prime} ; 1}^{a_{3}^{\prime}}\left(u,-x_{1}^{-1}\right) e^{x_{1} L(1)}\left(\left(e^{-\pi i} x_{1}^{-2}\right)^{L(0)} w_{a_{3}^{\prime}}, w_{a_{2}}\right\rangle \\
& =e^{\pi i\left(h_{a_{1}}+h_{a_{3}}\right)} . \\
& \cdot\left\langle e^{x_{1}^{-1} L(-1)} \sigma_{23}\left(\sigma_{123}\left(\mathcal{Y}_{a_{2} a_{3}^{\prime} ; i}^{a_{1}^{\prime}}\right)\right)\left(e^{x_{2} L(1)}\left(e^{-\pi i} x_{2}^{-2}\right)^{L(0)} w_{a_{1}}, x_{2}^{-1}-x_{1}^{-1}\right) \cdot\right. \\
& \left.\cdot Y_{W^{a_{3}^{\prime}}}\left(u,-x_{1}^{-1}\right) e^{x_{1} L(1)}\left(e^{-\pi i} x_{1}^{-2}\right)^{L(0)} w_{a_{3}^{\prime}}, w_{a_{2}}\right\rangle
\end{aligned}
$$

By the locality between vertex operators on $V$-modules and intertwining operators and the definition of $\sigma_{23}$, there exists a positive integer $N$ such that

$$
\begin{aligned}
& x_{2}^{-N} e^{\pi i\left(h_{a_{1}}+h_{a_{3}}\right)} \\
& \cdot\left\langle e^{x_{1}^{-1} L(-1)} \sigma_{23}\left(\sigma_{123}\left(\mathcal{Y}_{a_{2} a_{3}^{\prime} ; i}^{a^{\prime}}\right)\right)\left(e^{x_{2} L(1)}\left(e^{-\pi i} x_{2}^{-2}\right)^{L(0)} w_{a_{1}}, x_{2}^{-1}-x_{1}^{-1}\right) \cdot\right. \\
&\left.\cdot Y_{W_{3}^{a_{3}^{\prime}}}\left(u,-x_{1}^{-1}\right) e^{x_{1} L(1)}\left(e^{-\pi i} x_{1}^{-2}\right)^{L(0)} w_{a_{3}^{\prime}}, w_{a_{2}}\right\rangle\left(-x_{1}^{-1}\right)^{N} e^{\pi i\left(h_{a_{1}}+h_{a_{3}}\right)} \cdot \\
&=\left(\left(x_{2}^{-1}-x_{1}^{-1}\right)-\left(\mathcal{Y}_{a_{2} a_{3}^{\prime} ;}\right)\right)\left(e^{x_{2} L(1)}\left(e^{-\pi i} x_{2}^{-2}\right)^{L(0)} w_{a_{1}}, x_{2}^{-1}-x_{1}^{-1}\right) \cdot \\
& \cdot\left\langlee ^ { x _ { 1 } ^ { - 1 } L ( - 1 ) } \sigma _ { 2 3 } \left(\sigma_{123}\left(Y_{W_{3}^{a_{3}^{\prime}}}\left(u,-x_{1}^{-1}\right) e^{x_{1} L(1)}\left(e^{-\pi i} x_{1}^{-2}\right)^{L(0)} w_{a_{3}^{\prime}}, w_{a_{2}}\right\rangle\right.\right. \\
&=\left(\left(x_{2}^{-1}-x_{1}^{-1}\right)-\left(-x_{1}^{-1}\right)^{N} e^{\pi i\left(h_{a_{1}}+h_{a_{3}}\right)} \cdot\right. \\
& \cdot\left\langle e^{x_{1}^{-1} L(-1)} Y_{W_{a_{2}^{\prime}}}\left(u,-x_{1}^{-1}\right) \cdot\right. \\
& \cdot \sigma_{23}\left(\sigma_{123}\left(\mathcal{Y}_{a_{2} a_{3}^{\prime} ; i}^{a^{\prime}}\right)\right)\left(e^{x_{2} L(1)}\left(e^{-\pi i} x_{2}^{-2}\right)^{L(0)} w_{a_{1}}, z_{2}^{-1}-z_{1}^{-1}\right) \cdot \\
&\left.\cdot e^{z_{1} L(1)}\left(e^{-\pi i} z_{1}^{-2}\right)^{L(0)} w_{a_{3}^{\prime}}, w_{a_{2}}\right\rangle
\end{aligned}
$$




$$
\begin{aligned}
& \cdot\left\langle\sigma _ { 1 2 } ( Y _ { W _ { a _ { 2 } ^ { \prime } } } ) \left(\sigma _ { 2 3 } \left(\sigma_{123}\left(\mathcal{Y}_{a_{2} a_{3}^{\prime} ; i}^{a_{1}^{\prime}}\right)\left(e^{x_{2} L(1)}\left(e^{-\pi i} x_{2}^{-2}\right)^{L(0)} w_{a_{1}}, x_{2}^{-1}-x_{1}^{-1}\right) \cdot\right.\right.\right. \\
& \left.\left.\cdot e^{x_{1} L(1)}\left(e^{-\pi i} x_{1}^{-2}\right)^{L(0)} w_{a_{3}^{\prime}}, x_{1}^{-1}\right) u, w_{a_{2}}\right\rangle \\
& =x_{2}^{-N} e^{\pi i\left(h_{a_{1}}+h_{a_{3}}-h_{a_{2}}\right)}\left\langlee ^ { \pi i h _ { a _ { 1 } } } \mathcal { Y } _ { a _ { 2 } ^ { \prime } e ; 1 } ^ { a _ { 2 } ^ { \prime } } \left( e^{x_{1} L(1)}\left(e^{-\pi i} x_{1}^{-2}\right)^{L(0)}\left(e^{\pi i} x_{1}^{2}\right)^{L(0)} e^{-x_{1} L(1)} .\right.\right. \\
& \cdot \sigma_{23}\left(\sigma_{123}\left(\mathcal{Y}_{a_{2} a_{3}^{\prime} ; i}^{a^{\prime}}\right)\right)\left(e^{x_{2} L(1)}\left(e^{-\pi i} x_{2}^{-2}\right)^{L(0)} w_{a_{1}}, x_{2}^{-1}-x_{1}^{-1}\right) \cdot \\
& \left.\left.\cdot e^{x_{1} L(1)}\left(e^{-\pi i} x_{1}^{-2}\right)^{L(0)} w_{a_{3}^{\prime}}, x_{1}^{-1}\right) u, w_{a_{2}}\right\rangle \\
& =x_{2}^{-N} e^{\pi i\left(h_{a_{1}}+h_{a_{3}}-h_{a_{2}}\right)}\left\langle u, \sigma_{23}\left(\mathcal{Y}_{a_{2}^{\prime} e ; 1}^{a_{2}^{\prime}}\right)\left(\left(e^{\pi i} x_{1}^{2}\right)^{L(0)} e^{-x_{1} L(1)} .\right.\right. \\
& \cdot \sigma_{23}\left(\sigma_{123}\left(\mathcal{Y}_{a_{2} a_{3}^{\prime} ; i}^{a_{1}^{\prime}}\right)\right)\left(e^{x_{2} L(1)}\left(e^{-\pi i} x_{2}^{-2}\right)^{L(0)} w_{a_{1}}, x_{2}^{-1}-x_{1}^{-1}\right) \cdot \\
& \left.\left.\cdot e^{x_{1} L(1)}\left(e^{-\pi i} x_{1}^{-2}\right)^{L(0)} w_{a_{3}^{\prime}}, x_{1}\right) w_{a_{2}}\right\rangle \\
& =x_{2}^{-N} e^{\pi i\left(h_{a_{1}}+h_{a_{3}}-h_{a_{2}}\right)}\left\langle u, \mathcal{Y}_{a_{2}^{\prime} a_{2} ; 1}^{e}\left(\left(e^{-\pi i} x_{1}^{2}\right)^{L(0)} e^{-x_{1} L(1)}\right. \text {. }\right. \\
& \cdot \sigma_{23}\left(\sigma_{123}\left(\mathcal{Y}_{a_{2} a_{3}^{\prime} ; i}^{a^{\prime}}\right)\right)\left(e^{x_{2} L(1)}\left(e^{-\pi i} x_{2}^{-2}\right)^{L(0)} w_{a_{1}}, x_{2}^{-1}-x_{1}^{-1}\right) \cdot \\
& \left.\left.\cdot e^{x_{1} L(1)}\left(e^{-\pi i} x_{1}^{-2}\right)^{L(0)} w_{a_{3}^{\prime}}, x_{1}\right) w_{a_{2}}\right\rangle \\
& =x_{2}^{-N} e^{\pi i\left(h_{a_{1}}+h_{a_{3}}-h_{a_{2}}\right)} . \\
& \cdot\left\langle u, \mathcal{Y}_{a_{2}^{\prime} a_{2} ; 1}^{e}\left(\sigma_{23}\left(\sigma_{123}\left(\mathcal{Y}_{a_{2} a_{3}^{\prime} ;}^{a_{1}^{\prime}}\right)\right)\left(w_{a_{1}}, e^{\pi i}\left(x_{1}-x_{2}\right)\right) w_{a_{3}^{\prime}}, x_{1}\right) w_{a_{2}}\right\rangle \\
& =x_{2}^{-N}\left\langle u, \mathcal{Y}_{a_{2}^{\prime} a_{2} ; 1}^{e}\left(e^{\left(x_{2}-x_{1}\right) L(-1)} e^{-\pi i \Delta\left(\sigma_{23}\left(\sigma_{123}\left(\mathcal{Y}_{a_{2} a_{3}^{\prime} ; i}^{a_{1}^{\prime}}\right)\right)\right.} e^{\left(x_{1}-x_{2}\right) L(-1)}\right. \text {. }\right. \\
& \left.\left.\cdot \sigma_{23}\left(\sigma_{123}\left(\mathcal{Y}_{a_{2} a_{3}^{\prime} ; i}^{a_{1}^{\prime}}\right)\right)\left(w_{a_{1}}, e^{\pi i}\left(x_{1}-x_{2}\right)\right) w_{a_{3}^{\prime}}, z_{1}\right) w_{a_{2}}\right\rangle \\
& =x_{2}^{-N}\left\langle u, \mathcal{Y}_{a_{2}^{\prime} a_{2} ; 1}^{e}\left(e^{\left(x_{2}-x_{1}\right) L(-1)}\right. \text {. }\right. \\
& \left.\left.\cdot \sigma_{12}\left(\sigma_{23}\left(\sigma_{123}\left(\mathcal{Y}_{a_{2} a_{3}^{\prime} ; i}^{a_{1}^{\prime}}\right)\right)\right)\left(w_{a_{1}}, x_{1}-x_{2}\right) w_{a_{3}^{\prime}}, x_{1}\right) w_{a_{2}}\right\rangle .
\end{aligned}
$$

Using (4.5), (4.6) , the $L(-1)$-derivative property for $\sigma_{12}\left(\sigma_{23}\left(\sigma_{123}\left(\mathcal{Y}_{a_{2} a_{3}^{\prime} ; i}^{a_{1}^{\prime}}\right)\right)\right)$ and the equality $\sigma_{12} \sigma_{23}=\sigma_{123}$, we obtain

$$
\begin{aligned}
& E\left(\left\langle u, \mathcal{Y}_{a_{3}^{\prime} a_{3} ; 1}^{e}\left(w_{a_{3}^{\prime}}, z_{1}\right) \sigma_{123}\left(\mathcal{Y}_{a_{2} a_{3}^{\prime} ;}^{a_{1}^{\prime}}\right)\left(w_{a_{1}}, z_{2}\right) w_{a_{2}}\right\rangle\right) \\
& =E\left(\left\langleu, \mathcal{Y}_{a_{2}^{\prime} a_{2} ; 1}^{e}\left(e^{\left(z_{2}-z_{1}\right) L(-1)} .\right.\right.\right. \\
& \left.\left.\cdot \sigma_{12}\left(\sigma_{23}\left(\sigma_{123}\left(\mathcal{Y}_{a_{2} a_{3}^{\prime} ; i}^{a^{\prime}}\right)\right)\left(w_{a_{1}}, z_{1}-z_{2}\right) w_{a_{3}^{\prime}}, z_{1}\right) w_{a_{2}}\right\rangle\right) \\
& =E\left(\left\langle u, \mathcal{Y}_{a_{2}^{\prime} a_{2} ; 1}^{e}\left(\sigma_{123}^{2}\left(\mathcal{Y}_{a_{2} a_{3}^{\prime} ; i}^{a_{1}^{\prime}}\right)\left(w_{a_{1}}, z_{1}-z_{2}\right) w_{a_{3}^{\prime}}, z_{2}\right) w_{a_{2}}\right\rangle\right)
\end{aligned}
$$


Thus we obtain

$$
F\left(\mathcal{Y}_{a_{3}^{\prime} a_{3} ; 1}^{e} \otimes \sigma_{123}\left(\mathcal{Y}_{a_{2} a_{3}^{\prime} ; i}^{a_{1}^{\prime}}\right) ; \mathcal{Y}_{a_{2}^{\prime} a_{2} ; 1}^{e} \otimes \sigma_{123}^{2}\left(\mathcal{Y}_{a_{2} a_{3}^{\prime} ; n}^{a_{1}^{\prime}}\right)\right)=\delta_{i n} .
$$

Similarly, we can prove

$$
F\left(\mathcal{Y}_{a_{2} a_{2}^{\prime} ; 1}^{e} \otimes \sigma_{123}^{2}\left(\mathcal{Y}_{a_{2} a_{3}^{\prime} ; n}^{a_{1}^{\prime}}\right) ; \mathcal{Y}_{a_{1}^{\prime} a_{1} ; 1}^{e} \otimes \mathcal{Y}_{a_{2} a_{3}^{\prime} ; j}^{a_{1}^{\prime}}\right)=\delta_{n j}
$$

Using (4.7) and (4.8), we see that (4.4) becomes

$$
\begin{gathered}
\sum_{k=1}^{N_{a_{1}^{\prime} a_{3}}^{a_{2}}} F\left(\mathcal{Y}_{a_{2} e ; 1}^{a_{2}} \otimes \mathcal{Y}_{a_{3}^{\prime} a_{3} ; 1}^{e} ; \mathcal{Y}_{a_{1}^{\prime} a_{3} ; k}^{a_{2}} \otimes \mathcal{Y}_{a_{2} a_{3}^{\prime} ; j}^{a_{1}^{\prime}}\right) \\
\cdot F\left(\mathcal{Y}_{a_{1}^{\prime} a_{3} ; k}^{a_{2}} \otimes \sigma_{123}\left(\mathcal{Y}_{a_{2} a_{3}^{\prime} ; i}^{a_{1}^{\prime}}\right) ; \mathcal{Y}_{e a_{2} ; 1}^{a_{2}} \otimes \mathcal{Y}_{a_{1}^{\prime} a_{1} ; 1}^{e}\right) \\
=\delta_{i j} F\left(\mathcal{Y}_{a_{2} e ; 1}^{a_{2}} \otimes \mathcal{Y}_{a_{2}^{\prime} a_{2} ; 1}^{e} ; \mathcal{Y}_{e a_{2} ; 1}^{a_{2}} \otimes \mathcal{Y}_{a_{2} a_{2}^{\prime} ; 1}^{e}\right) .
\end{gathered}
$$

Summing over $i=1, \ldots, \mathbb{N}_{a_{1} a_{3}}^{a_{2}}$ on both sides in the special case $j=i$ of (4.9), we obtain (4.1).

We now prepare to prove the second formula. Recall from Section 2 the $\operatorname{maps} \Psi_{a_{1}, a_{2}, e}^{1,1}: \coprod_{a \in \mathcal{A}} W^{a} \otimes W^{a^{\prime}} \rightarrow \mathbb{G}_{1 ; 2}^{e}$ for $a_{1}, a_{2} \in \mathcal{A}$ and the projection $\pi:$ $\mathcal{F}_{1 ; 2} \rightarrow \mathcal{F}_{1 ; 2}^{e}$. For any $f \in \mathcal{F}_{1 ; 2}$, we shall, for convenience, denote $(\pi(f))\left(w_{a} \otimes\right.$ $\left.w_{a^{\prime}}\right)$ by $\pi\left(f\left(w_{a} \otimes w_{a^{\prime}}\right)\right)$.

We need the following lemma:

Lemma 4.2 For $a_{1}, a_{2} \in \mathcal{A}, w_{a_{2}} \in W^{a_{2}}$ and $w_{a_{2}^{\prime}} \in W^{a_{2}^{\prime}}$, we have

$$
\begin{gathered}
\Psi_{a_{1}, a_{2}, e}^{1,1}\left(w_{a_{2}}, w_{a_{2}^{\prime}} ; z_{1}, z_{2}-1 ; \tau\right) \\
=\sum_{a_{3} \in \mathcal{A}} \sum_{i=1}^{N_{a_{2} a_{3}}^{a_{1}}} \sum_{j=1}^{N_{a_{2}^{\prime} a_{1}}^{a_{3}}} \sum_{a_{4} \in \mathcal{A}} \sum_{k=1}^{N_{a_{4} a_{1}}^{a_{1}}} \sum_{l=1}^{N_{a_{2} a_{2}^{\prime}}^{a_{4}}} e^{-2 \pi i\left(h_{a_{3}}-h_{a_{1}}\right)} . \\
\cdot F^{-1}\left(\mathcal{Y}_{e a_{1} ; 1}^{a_{1}} \otimes \mathcal{Y}_{a_{2} a_{2}^{\prime} ; 1}^{e} ; \mathcal{Y}_{a_{2} a_{3} ; i}^{a_{1}} \otimes \mathcal{Y}_{a_{2}^{\prime} a_{1} ; j}^{a_{3}}\right) \cdot \\
\cdot F\left(\mathcal{Y}_{a_{2} a_{3} ; i}^{a_{1}} \otimes \mathcal{Y}_{a_{2}^{\prime} a_{1} ; j}^{a_{3}} ; \mathcal{Y}_{a_{4} a_{1} ; k}^{a_{1}} \otimes \mathcal{Y}_{a_{2} a_{2}^{\prime} ; l}^{a_{4}}\right) \cdot \\
\cdot E\left(\operatorname { T r } _ { W ^ { a _ { 1 } } { } _ { 1 } } \mathcal { Y } _ { a _ { 4 } a _ { 1 } ; k } ^ { a _ { 1 } } \left(\mathcal{U}\left(e^{2 \pi i z_{2}}\right) \cdot\right.\right. \\
\left.\left.\cdot \mathcal{Y}_{a_{2} a_{2}^{\prime} ; l}^{a_{4}}\left(w_{a_{2}}, z_{1}-z_{2}\right) w_{a_{2}^{\prime}}, e^{2 \pi i z_{2}}\right) q_{\tau}^{L(0)-\frac{c}{24}}\right)
\end{gathered}
$$


and

$$
\begin{aligned}
& \Psi_{a_{1}, a_{2}, e}^{1,1}\left(w_{a_{2}}, w_{a_{2}^{\prime}} ; z_{1}, z_{2}+\tau ; \tau\right) \\
& =\sum_{a_{3} \in \mathcal{A}} \sum_{i=1}^{N_{a_{2} a_{3}}^{a_{1}}} \sum_{j=1}^{N_{a_{2}^{\prime} a_{1}}^{a_{3}}} \sum_{a_{4} \in \mathcal{A}} \sum_{k=1}^{N_{a_{4} a_{3}}^{a_{3}}} \sum_{l=1}^{N_{a_{2} a_{2}^{\prime}}^{a_{4}}} e^{\pi i\left(-2 h_{a_{2}}+h_{a_{4}}\right)} \\
& F^{-1}\left(\mathcal{Y}_{e a_{1} ; 1}^{a_{1}} \otimes \mathcal{Y}_{a_{2} a_{2}^{\prime} ; 1}^{e} ; \sigma_{23}\left(\mathcal{Y}_{a_{2} a_{1}^{\prime} ; i}^{a^{\prime}}\right) \otimes \sigma_{13}\left(\mathcal{Y}_{a_{3}^{\prime} a_{1} ; j}^{a_{2}}\right)\right) \cdot \\
& \cdot F\left(\mathcal{Y}_{a_{2} a_{1}^{\prime} ; i}^{a_{\prime}^{\prime}} \otimes \sigma_{123}\left(\mathcal{Y}_{a_{3}^{\prime} a_{1} ; j}^{a_{2}}\right) ; \mathcal{Y}_{a_{4} a_{3}^{\prime} ; k}^{a_{\prime}^{\prime}} \otimes \mathcal{Y}_{a_{2} a_{2}^{\prime} ; l}^{a_{4}}\right) \cdot \\
& \cdot E\left(\operatorname{Tr}_{W^{a_{3}}} \mathcal{Y}_{a_{4} a_{3} ; k}^{a_{3}}\left(\mathcal{U}\left(e^{2 \pi i z_{2}}\right) \mathcal{Y}_{a_{2} a_{2}^{\prime} ; l}^{a_{4}}\left(w_{a_{2}}, z_{1}-z_{2}\right) w_{a_{2}^{\prime}}, e^{2 \pi i z_{2}}\right) q_{\tau}^{L(0)-\frac{c}{24}}\right) .
\end{aligned}
$$

In particular, for any $a_{1}, a_{2} \in \mathcal{A}$, the maps from $\coprod_{a \in \mathcal{A}} W^{a} \otimes W^{a^{\prime}}$ to the space of single-valued analytic functions on $\widetilde{M}_{1}^{2}$ given by

$$
\begin{aligned}
w_{a} \otimes w_{a^{\prime}} & \mapsto \Psi_{a_{1}, a_{2}, e}^{1,1}\left(w_{a}, w_{a^{\prime}} ; z_{1}, z_{2}-1 ; \tau\right), \\
w_{a} \otimes w_{a^{\prime}} & \mapsto \Psi_{a_{1}, a_{2}, e}^{1,1}\left(w_{a}, w_{a^{\prime}} ; z_{1}, z_{2}+\tau ; \tau\right)
\end{aligned}
$$

for $w_{a} \in W^{a}$ and $w_{a^{\prime}} \in W^{a^{\prime}}$ are in $\mathcal{F}_{1 ; 2}$.

Proof. Using the definition of $\Psi_{a_{1}, a_{2}, e}^{1,1}$, the associativity properties (1.5), (1.6) and (1.1), we have

$$
\begin{aligned}
& \Psi_{a_{1}, a_{2}, e}^{1,1}\left(w_{a_{2}}, w_{a_{2}^{\prime}} ; z_{1}, z_{2}-1 ; \tau\right) \\
& =E\left(\operatorname { T r } _ { W ^ { a _ { 1 } } } \mathcal { Y } _ { e a _ { 1 } ; 1 } ^ { a _ { 1 } } \left(\mathcal{U}\left(e^{2 \pi i\left(z_{2}-1\right)}\right)\right.\right. \text {. } \\
& \left.\left.\cdot \mathcal{Y}_{a_{2} a_{2}^{\prime} ; 1}^{e}\left(w_{a_{2}}, z_{1}-\left(z_{2}-1\right)\right) w_{a_{2}^{\prime}}, e^{2 \pi i\left(z_{2}-1\right)}\right) q_{\tau}^{L(0)-\frac{c}{24}}\right)
\end{aligned}
$$

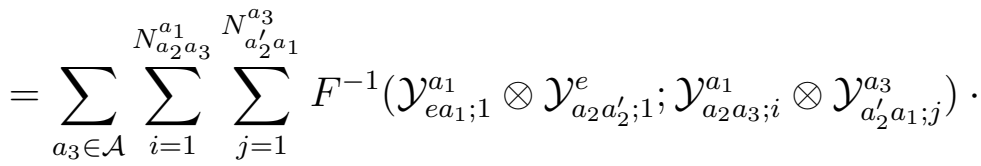

$$
\begin{aligned}
& \cdot E\left(\operatorname{Tr}_{W^{a_{1}}} \mathcal{Y}_{a_{2} a_{3} ; i}^{a_{1}}\left(\mathcal{U}\left(e^{2 \pi i z_{1}}\right) w_{a_{2}}, e^{2 \pi i z_{1}}\right) \cdot\right. \\
& \left.\cdot \mathcal{Y}_{a_{2}^{\prime} a_{1} ; j}^{a_{3}}\left(\mathcal{U}\left(e^{2 \pi i\left(z_{2}-1\right)}\right) w_{a_{2}^{\prime}}, e^{2 \pi i\left(z_{2}-1\right)}\right) q_{\tau}^{L(0)-\frac{c}{24}}\right) \\
& =\sum_{a_{3} \in \mathcal{A}} \sum_{i=1}^{N_{a_{2} a_{3}}^{a_{1}}} \sum_{j=1}^{N_{a_{2}^{\prime} a_{1}}^{a_{3}}} F^{-1}\left(\mathcal{Y}_{e a_{1} ; 1}^{a_{1}} \otimes \mathcal{Y}_{a_{2} a_{2}^{\prime} ; 1}^{e} ; \mathcal{Y}_{a_{2} a_{3} ; i}^{a_{1}} \otimes \mathcal{Y}_{a_{2}^{\prime} a_{1} ; j}^{a_{3}}\right) \text {. }
\end{aligned}
$$




$$
\begin{aligned}
& \cdot E\left(\operatorname{Tr}_{W^{a_{1}}} \mathcal{Y}_{a_{2} a_{3} ; i}^{a_{1}}\left(\mathcal{U}\left(e^{2 \pi i z_{1}}\right) w_{a_{2}}, e^{2 \pi i z_{1}}\right) \cdot\right. \\
& \left.\cdot \mathcal{Y}_{a_{2}^{\prime} a_{1} ; j}^{a_{3}}\left(\mathcal{U}\left(e^{-2 \pi i} e^{2 \pi i z_{2}}\right) w_{a_{2}^{\prime}}, e^{-2 \pi i} e^{2 \pi i z_{2}}\right) q_{\tau}^{L(0)-\frac{c}{24}}\right) \\
& =\sum_{a_{3} \in \mathcal{A}} \sum_{i=1}^{N_{a_{2} a_{3}}^{a_{1}}} \sum_{j=1}^{N_{a_{2}^{\prime} a_{1}}^{a_{3}}} e^{-2 \pi i\left(h_{a_{3}}-h_{a_{1}}\right)} F^{-1}\left(\mathcal{Y}_{e a_{1} ; 1}^{a_{1}} \otimes \mathcal{Y}_{a_{2} a_{2}^{\prime} ; 1}^{e} ; \mathcal{Y}_{a_{2} a_{3} ; i}^{a_{1}} \otimes \mathcal{Y}_{a_{2}^{\prime} a_{1} ; j}^{a_{3}}\right) \text {. } \\
& \cdot E\left(\operatorname{Tr}_{W^{a_{1}}} \mathcal{Y}_{a_{2} a_{3} ; i}^{a_{1}}\left(\mathcal{U}\left(e^{2 \pi i z_{1}}\right) w_{a_{2}}, e^{2 \pi i z_{1}}\right) \cdot\right. \\
& \left.\cdot \mathcal{Y}_{a_{2}^{\prime} a_{1} ; j}^{a_{3}}\left(\mathcal{U}\left(e^{2 \pi i z_{2}}\right) w_{a_{2}^{\prime}}, e^{2 \pi i z_{2}}\right) q_{\tau}^{L(0)-\frac{c}{24}}\right) \\
& =\sum_{a_{3} \in \mathcal{A}} \sum_{i=1}^{N_{a_{2} a_{3}}^{a_{1}}} \sum_{j=1}^{N_{a_{2}^{\prime} a_{1}}^{a_{3}}} e^{-2 \pi i\left(h_{a_{3}}-h_{a_{1}}\right)} F^{-1}\left(\mathcal{Y}_{e a_{1} ; 1}^{a_{1}} \otimes \mathcal{Y}_{a_{2} a_{2}^{\prime} ; 1}^{e} ; \mathcal{Y}_{a_{2} a_{3} ; i}^{a_{1}} \otimes \mathcal{Y}_{a_{2}^{\prime} a_{1} ; j}^{a_{3}}\right) \text {. }
\end{aligned}
$$

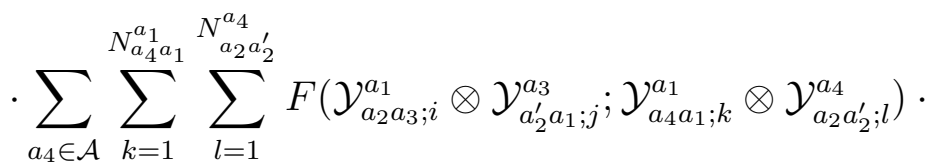

$$
\begin{aligned}
& \cdot E\left(\operatorname { T r } _ { W ^ { a _ { 1 } } } \mathcal { Y } _ { a _ { 4 } a _ { 1 } ; k } ^ { a _ { 1 } } \left(\mathcal{U}\left(e^{2 \pi i z_{2}}\right) \cdot\right.\right. \\
& \left.\left.\cdot \mathcal{Y}_{a_{2} a_{2}^{\prime} ; l}^{a_{4}}\left(w_{a_{2}}, z_{1}-z_{2}\right) w_{a_{2}^{\prime}}, e^{2 \pi i z_{2}}\right) q_{\tau}^{L(0)-\frac{c}{24}}\right),
\end{aligned}
$$

proving (4.10).

The proof of (4.11) is more complicated. Using the definition of $\Psi_{a_{1}, a_{2}, e}^{1,1}$, the associativity properties (1.5), (1.6), the $L(0)$-conjugation property, the property of traces, and (1.1),

$$
\begin{aligned}
& \Psi_{a_{1}, a_{2}, e}^{1,1}\left(w_{a_{2}}, w_{a_{2}^{\prime}} ; z_{1}, z_{2}+\tau ; \tau\right) \\
& =E\left(\operatorname { T r } _ { W ^ { a _ { 1 } } } \mathcal { Y } _ { e a _ { 1 } ; 1 } ^ { a _ { 1 } } \left(\mathcal{U}\left(e^{2 \pi i\left(z_{2}+\tau\right)}\right)\right.\right. \text {. } \\
& \left.\left.\cdot \mathcal{Y}_{a_{2} a_{2}^{\prime} ; 1}^{e}\left(w_{a_{2}}, z_{1}-\left(z_{2}+\tau\right)\right) w_{a_{2}^{\prime}}, e^{2 \pi i\left(z_{2}+\tau\right)}\right) q_{\tau}^{L(0)-\frac{c}{24}}\right) \\
& =\sum_{a_{3} \in \mathcal{A}} \sum_{i=1}^{N_{a_{2} a_{3}}^{a_{1}}} \sum_{j=1}^{N_{a_{2}^{\prime} a_{1}}^{a_{3}}} F^{-1}\left(\mathcal{Y}_{e a_{1} ; 1}^{a_{1}} \otimes \mathcal{Y}_{a_{2} a_{2}^{\prime} ; 1}^{e} ; \sigma_{23}\left(\mathcal{Y}_{a_{2} a_{1}^{\prime} ; i}^{a_{3}^{\prime}}\right) \otimes \sigma_{13}\left(\mathcal{Y}_{a_{3}^{\prime} a_{1} ; j}^{a_{2}}\right)\right) \text {. } \\
& \cdot E\left(\operatorname{Tr}_{W^{a_{1}}} \sigma_{23}\left(\mathcal{Y}_{a_{2} a_{1}^{\prime} ; i}^{a_{3}^{\prime}}\right)\left(\mathcal{U}\left(e^{2 \pi i z_{1}}\right) w_{a_{2}}, e^{2 \pi i z_{1}}\right) \cdot\right. \\
& \left.\cdot \sigma_{13}\left(\mathcal{Y}_{a_{3}^{\prime} a_{1} ; j}^{a_{2}}\right)\left(\mathcal{U}\left(e^{2 \pi i\left(z_{2}+\tau\right)}\right) w_{a_{2}^{\prime}}, e^{2 \pi i\left(z_{2}+\tau\right)}\right) q_{\tau}^{L(0)-\frac{c}{24}}\right)
\end{aligned}
$$




$$
\begin{aligned}
& =\sum_{a_{3} \in \mathcal{A}} \sum_{i=1}^{N_{a_{2} a_{3}}^{a_{1}}} \sum_{j=1}^{N_{a_{2}^{\prime} a_{1}}^{a_{3}}} F^{-1}\left(\mathcal{Y}_{e a_{1} ; 1}^{a_{1}} \otimes \mathcal{Y}_{a_{2} a_{2}^{\prime} ; 1}^{e} ; \sigma_{23}\left(\mathcal{Y}_{a_{2} a_{1}^{\prime} ; i}^{a_{3}^{\prime}}\right) \otimes \sigma_{13}\left(\mathcal{Y}_{a_{3}^{\prime} a_{1} ; j}^{a_{2}}\right)\right) \\
& \cdot E\left(\operatorname{Tr}_{W^{a_{1}} \sigma_{23}}\left(\mathcal{Y}_{a_{2} a_{1}^{\prime} ; i}^{a_{3}^{\prime}}\right)\left(\mathcal{U}\left(e^{2 \pi i z_{1}}\right) w_{a_{2}}, e^{2 \pi i z_{1}}\right) \cdot\right. \\
& \left.\cdot \sigma_{13}\left(\mathcal{Y}_{a_{3}^{\prime} a_{1} ; j}^{a_{2}}\right)\left(\mathcal{U}\left(q_{\tau} e^{2 \pi i z_{2}}\right) w_{a_{2}^{\prime}}, q_{\tau} e^{2 \pi i z_{2}}\right) q_{\tau}^{L(0)-\frac{c}{24}}\right) \\
& =\sum_{a_{3} \in \mathcal{A}} \sum_{i=1}^{N_{a_{2} a_{3}}^{a_{1}}} \sum_{j=1}^{N_{a_{2}^{\prime} a_{1}}^{a_{3}}} F^{-1}\left(\mathcal{Y}_{e a_{1} ; 1}^{a_{1}} \otimes \mathcal{Y}_{a_{2} a_{2}^{\prime} ; 1}^{e} ; \sigma_{23}\left(\mathcal{Y}_{a_{2} a_{1}^{\prime} ; i}^{a_{3}^{\prime}}\right) \otimes \sigma_{13}\left(\mathcal{Y}_{a_{3}^{\prime} a_{1} ; j}^{a_{2}}\right)\right) \text {. } \\
& \cdot E\left(\operatorname{Tr}_{W^{a_{1}}} \sigma_{23}\left(\mathcal{Y}_{a_{2} a_{1}^{\prime} ; i}^{a_{3}^{\prime}}\right)\left(\mathcal{U}\left(e^{2 \pi i z_{1}}\right) w_{a_{2}}, e^{2 \pi i z_{1}}\right) \cdot\right. \\
& \left.\cdot q_{\tau}^{L(0)-\frac{c}{24}} \sigma_{13}\left(\mathcal{Y}_{a_{3}^{\prime} a_{1} ; j}^{a_{2}}\right)\left(\mathcal{U}\left(e^{2 \pi i z_{2}}\right) w_{a_{2}^{\prime}}, e^{2 \pi i z_{2}}\right)\right) \\
& =\sum_{a_{3} \in \mathcal{A}} \sum_{i=1}^{N_{a_{2} a_{3}}^{a_{1}}} \sum_{j=1}^{N_{a_{2}^{\prime} a_{1}}^{a_{3}}} F^{-1}\left(\mathcal{Y}_{e a_{1} ; 1}^{a_{1}} \otimes \mathcal{Y}_{a_{2} a_{2}^{\prime} ; 1}^{e} ; \sigma_{23}\left(\mathcal{Y}_{a_{2} a_{1}^{\prime} ; i}^{a_{3}^{\prime}}\right) \otimes \sigma_{13}\left(\mathcal{Y}_{a_{3}^{\prime} a_{1} ; j}^{a_{2}}\right)\right) \text {. } \\
& \cdot E\left(\operatorname{Tr}_{W^{a_{3}} \sigma_{13}}\left(\mathcal{Y}_{a_{3}^{\prime} a_{1} ; j}^{a_{2}}\right)\left(\mathcal{U}\left(e^{2 \pi i z_{2}}\right) w_{a_{2}^{\prime}}, e^{2 \pi i z_{2}}\right) \cdot\right. \\
& \left.\cdot \sigma_{23}\left(\mathcal{Y}_{a_{2} a_{1}^{\prime} ; i}^{a_{3}^{\prime}}\right)\left(\mathcal{U}\left(e^{2 \pi i z_{1}}\right) w_{a_{2}}, e^{2 \pi i z_{1}}\right) q_{\tau}^{L(0)-\frac{c}{24}}\right) .
\end{aligned}
$$

Using (2.21), the relations $\sigma_{23}^{2}=1, \sigma_{23} \sigma_{13}=\sigma_{123}$ and the genus-one associativity, we have

$$
\begin{aligned}
& E\left(\operatorname{Tr}_{W^{a_{3}}} \sigma_{13}\left(\mathcal{Y}_{a_{3}^{\prime} a_{1} ; j}^{a_{2}}\right)\left(\mathcal{U}\left(e^{2 \pi i z_{2}}\right) w_{a_{2}^{\prime}}, e^{2 \pi i z_{2}}\right) .\right. \\
& \left.\cdot \sigma_{23}\left(\mathcal{Y}_{a_{2} a_{1}^{\prime} ; i}^{a_{3}^{\prime}}\right)\left(\mathcal{U}\left(e^{2 \pi i z_{1}}\right) w_{a_{2}}, e^{2 \pi i z_{1}}\right) q_{\tau}^{L(0)-\frac{c}{24}}\right) \\
& =E\left(\operatorname{Tr}_{W^{a_{3}}} \sigma_{23}^{2}\left(\sigma_{13}\left(\mathcal{Y}_{a_{3}^{\prime} a_{1} ; j}^{a_{2}}\right)\right)\left(\mathcal{U}\left(e^{2 \pi i z_{2}}\right) w_{a_{2}^{\prime}}, e^{2 \pi i z_{2}}\right) .\right. \\
& \left.\cdot \sigma_{23}\left(\mathcal{Y}_{a_{2} a_{1}^{\prime} ; i}^{a_{3}^{\prime}}\right)\left(\mathcal{U}\left(e^{2 \pi i z_{1}}\right) w_{a_{2}}, e^{2 \pi i z_{1}}\right) q_{\tau}^{L(0)-\frac{c}{24}}\right) \\
& =e^{-2 \pi i h_{a_{2}}} E\left(\operatorname{Tr}_{W^{a_{3}^{\prime}}} \mathcal{Y}_{a_{2} a_{1}^{\prime} ; i}^{a^{\prime}}\left(\mathcal{U}\left(e^{2 \pi i z_{1}}\right) e^{\pi i L(0)} w_{a_{2}}, e^{-2 \pi i z_{1}}\right) .\right. \\
& \left.\cdot \sigma_{23}\left(\sigma_{13}\left(\mathcal{Y}_{a_{3}^{\prime} a_{1} ; j}^{a_{2}}\right)\right)\left(\mathcal{U}\left(e^{2 \pi i z_{2}}\right) e^{\pi i L(0)} w_{a_{2}^{\prime}}, e^{-2 \pi i z_{2}}\right) q_{\tau}^{L(0)-\frac{c}{24}}\right) \\
& =e^{-2 \pi i h_{a_{2}}} E\left(\operatorname{Tr}_{W^{a_{3}^{\prime}}} \mathcal{Y}_{a_{2} a_{1}^{\prime} ; i}^{a_{3}^{\prime}}\left(\mathcal{U}\left(e^{-2 \pi i z_{1}}\right) e^{\pi i L(0)} w_{a_{2}}, e^{-2 \pi i z_{1}}\right)\right. \text {. } \\
& \left.\cdot \sigma_{123}\left(\mathcal{Y}_{a_{3}^{\prime} a_{1} ; j}^{a_{2}}\right)\left(\mathcal{U}\left(e^{-2 \pi i z_{2}}\right) e^{\pi i L(0)} w_{a_{2}^{\prime}}, e^{-2 \pi i z_{2}}\right) q_{\tau}^{L(0)-\frac{c}{24}}\right)
\end{aligned}
$$


We now prove

$$
\begin{aligned}
& E\left(\operatorname{Tr}_{W^{a_{3}^{\prime}}} \mathcal{Y}_{a_{2} a_{1}^{\prime} ; i}^{a^{\prime}}\left(\mathcal{U}\left(e^{-2 \pi i z_{1}}\right) e^{\pi i L(0)} w_{a_{2}}, e^{-2 \pi i z_{1}}\right)\right. \\
& \left.\cdot \sigma_{123}\left(\mathcal{Y}_{a_{3}^{\prime} a_{1} ; j}^{a_{2}}\right)\left(\mathcal{U}\left(e^{-2 \pi i z_{2}}\right) e^{\pi i L(0)} w_{a_{2}^{\prime}}, e^{-2 \pi i z_{2}}\right) q_{\tau}^{L(0)-\frac{c}{24}}\right)
\end{aligned}
$$

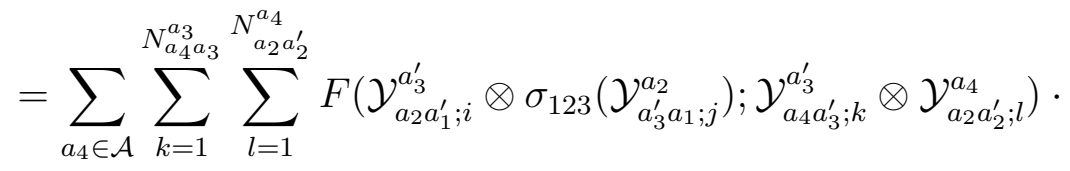

$$
\begin{aligned}
& \cdot E\left(\operatorname { T r } _ { W ^ { a _ { 3 } ^ { \prime } } } \mathcal { Y } _ { a _ { 4 } a _ { 3 } ^ { \prime } ; k } ^ { a _ { 3 } ^ { \prime } } \left(\mathcal{U}\left(e^{-2 \pi i z_{2}}\right) \mathcal{Y}_{a_{2} a_{2}^{\prime} ; l}^{a_{4}}\left(e^{\pi i L(0)} w_{a_{2}}, e^{\pi i}\left(z_{1}-z_{2}\right)\right) .\right.\right. \\
& \left.\left.\cdot e^{\pi i L(0)} w_{a_{2}^{\prime}}, e^{-2 \pi i z_{2}}\right) q_{\tau}^{L(0)-\frac{c}{24}}\right) .
\end{aligned}
$$

To prove (4.14), we need only prove their restrictions to a subregion of $\widetilde{M}_{1}^{2}$ are equal. So we need only prove that

$$
\begin{array}{r}
\operatorname{Tr}_{W^{a_{3}^{\prime}}} \mathcal{Y}_{a_{2} a_{1}^{\prime} ; i}^{a_{3}^{\prime}}\left(\mathcal{U}\left(e^{-2 \pi i z_{1}}\right) e^{\pi i L(0)} w_{a_{2}}, e^{-2 \pi i z_{1}}\right) \cdot \\
\cdot \sigma_{123}\left(\mathcal{Y}_{a_{3}^{\prime} a_{1} ; j}^{a_{2}}\right)\left(\mathcal{U}\left(e^{-2 \pi i z_{2}}\right) e^{\pi i L(0)} w_{a_{2}^{\prime}}, e^{-2 \pi i z_{2}}\right) q_{\tau}^{L(0)-\frac{c}{24}} \\
=\sum_{a_{4} \in \mathcal{A}} \sum_{k=1}^{N_{a_{4} a_{3}}^{a_{3}}} \sum_{l=1}^{N_{a_{2} a_{2}^{\prime}}^{a_{4}}} F\left(\mathcal{Y}_{a_{2} a_{1}^{\prime} ; i}^{a_{3}^{\prime}} \otimes \sigma_{123}\left(\mathcal{Y}_{a_{3}^{\prime} a_{1} ; j}^{a_{2}}\right) ; \mathcal{Y}_{a_{4} a_{3}^{\prime} ; k}^{a_{3}^{\prime}} \otimes \mathcal{Y}_{a_{2} a_{2}^{\prime} ; l}^{a_{4}}\right) \cdot \\
\cdot \operatorname{Tr}_{W^{a_{3}^{\prime}}} \mathcal{Y}_{a_{4} a_{3}^{\prime} ; k}^{a_{3}^{\prime}}\left(\mathcal{U}\left(e^{-2 \pi i z_{2}}\right) \mathcal{Y}_{a_{2} a_{2}^{\prime} ; l}^{a_{4}}\left(e^{\pi i L(0)} w_{a_{2}}, e^{\pi i}\left(z_{1}-z_{2}\right)\right) \cdot\right. \\
\left.\left.\cdot e^{\pi i L(0)} w_{a_{2}^{\prime}}, e^{-2 \pi i z_{2}}\right) q_{\tau}^{L(0)-\frac{c}{24}}\right)
\end{array}
$$

holds when $\left|q_{\tau}\right|<\left|e^{-2 \pi i z_{2}}\right|<\left|e^{-2 \pi i z_{1}}\right|<1$ and $0<\left|e^{2 \pi i\left(-z_{1}+z_{2}\right)}-1\right|<1$. From (2.9), we see that in this region the left-hand side of (4.15) is equal to

$$
\begin{aligned}
\sum_{a_{4} \in \mathcal{A}} \sum_{k=1}^{N_{a_{4} a_{3}}^{a_{3}}} \sum_{l=1}^{N_{a_{2} a_{2}^{\prime}}^{a_{4}} F\left(\mathcal{Y}_{a_{2} a_{1}^{\prime} ; i}^{a_{3}^{\prime}} \otimes \sigma_{123}\left(\mathcal{Y}_{a_{3}^{\prime} a_{1} ; j}^{a_{2}}\right) ; \mathcal{Y}_{a_{4} a_{3}^{\prime} ; k}^{a_{3}^{\prime}} \otimes \mathcal{Y}_{a_{2} a_{2}^{\prime} ; l}^{a_{4}}\right) .} \\
\cdot \operatorname{Tr}_{W^{a_{3}^{\prime}}} \mathcal{Y}_{a_{4} a_{3}^{\prime} ; k}^{a_{3}^{\prime}}\left(\mathcal{U}\left(e^{-2 \pi i z_{2}}\right) \mathcal{Y}_{a_{2} a_{2}^{\prime} ; l}^{a_{4}}\left(e^{\pi i L(0)} w_{a_{2}},\left(-z_{1}+z_{2}\right)\right) .\right.
\end{aligned}
$$




$$
\left.\left.\cdot e^{\pi i L(0)} w_{a_{2}^{\prime}}, e^{-2 \pi i z_{2}}\right) q_{\tau}^{L(0)-\frac{c}{24}}\right)
$$

Now in this region, because $\left|e^{-2 \pi i z_{2}}\right|<\left|e^{-2 \pi i z_{1}}\right|$, the imaginary part of $z_{1}$ must be bigger than the imaginary part of $z_{2}$. Thus $z_{1}-z_{2}$ is in the upper half plane. This means that $\arg \left(z_{1}-z_{2}\right)<\pi$ and $\arg \left(z_{1}-z_{2}\right)+\pi<2 \pi$. So we have $\arg \left(-\left(z_{1}-z_{2}\right)\right)=\arg \left(z_{1}-z_{2}\right)+\pi$. Now for any $n \in \mathbb{C}$, by our convention,

$$
\begin{aligned}
\left(-z_{1}+z_{2}\right)^{n} & =e^{n \log \left(-z_{1}+z_{2}\right)} \\
& =e^{n \log \left(-\left(z_{1}-z_{2}\right)\right)} \\
& =e^{n\left(\log \left|-\left(z_{1}-z_{2}\right)\right|+i \arg \left(-\left(z_{1}-z_{2}\right)\right)\right)} \\
& =e^{n\left(\log \left|\left(z_{1}-z_{2}\right)\right|+i \arg \left(z_{1}-z_{2}\right)+\pi i\right)} \\
& =e^{n\left(\log \left(z_{1}-z_{2}\right)+\pi i\right)} \\
& =\left(e^{\pi i}\left(z_{1}-z_{2}\right)\right)^{n}
\end{aligned}
$$

This shows that indeed when $\left|q_{\tau}\right|<\left|e^{-2 \pi i z_{2}}\right|<\left|e^{-2 \pi i z_{1}}\right|<1$ and $0<$ $\left|e^{2 \pi i\left(-z_{1}+z_{2}\right)}-1\right|<1$, (4.16) is equal to the right-hand side of (4.15) and (4.15) holds. Consequently, we obtain (4.14).

Using (2.22) and the $L(0)$-conjugation formula, we have

$$
\begin{gathered}
E\left(\operatorname { T r } _ { W ^ { a _ { 3 } ^ { \prime } } } \mathcal { Y } _ { a _ { 4 } a _ { 3 } ^ { \prime } ; k } ^ { a _ { 3 } ^ { \prime } } \left(\mathcal{U}\left(e^{-2 \pi i z_{2}}\right) \mathcal{Y}_{a_{2} a_{2}^{\prime} ; l}^{a_{4}}\left(e^{\pi i L(0)} w_{a_{2}}, e^{\pi i}\left(z_{1}-z_{2}\right)\right) \cdot\right.\right. \\
\left.\left.\cdot e^{\pi i L(0)} w_{a_{2}^{\prime}}, e^{-2 \pi i z_{2}}\right) q_{\tau}^{L(0)-\frac{c}{24}}\right) \\
=e^{\pi h_{a_{4}}} E\left(\operatorname { T r } _ { W ^ { a _ { 3 } } } \mathcal { Y } _ { a _ { 4 } a _ { 3 } ; k } ^ { a _ { 3 } } \left(\mathcal{U}\left(e^{2 \pi i z_{2}}\right) e^{-\pi i L(0)} \cdot\right.\right. \\
\left.\left.\cdot \mathcal{Y}_{a_{2} a_{2}^{\prime} ; l}^{a_{4}}\left(e^{\pi i L(0)} w_{a_{2}}, e^{\pi i}\left(z_{1}-z_{2}\right)\right) e^{\pi i L(0)} w_{a_{2}^{\prime}}, e^{2 \pi i z_{2}}\right) q_{\tau}^{L(0)-\frac{c}{24}}\right) \\
=e^{\pi h_{a_{4}}} E\left(\operatorname{Tr}_{W^{a_{3}}} \mathcal{Y}_{a_{4} a_{3} ; k}^{a_{3}}\left(\mathcal{U}\left(e^{2 \pi i z_{2}}\right) \mathcal{Y}_{a_{2} a_{2}^{\prime} ; l}^{a_{4}}\left(w_{a_{2}}, z_{1}-z_{2}\right) w_{a_{2}^{\prime}}, e^{2 \pi i z_{2}}\right) q_{\tau}^{L(0)-\frac{c}{24}}\right) .
\end{gathered}
$$

Combining (4.12), (4.13), (4.14) and (4.17), we obtain (4.11).

For $a_{1}, a_{2} \in \mathcal{A}$, we define $\alpha\left(\Psi_{a_{1}, a_{2}, e}^{1,1}\right)$ and $\beta\left(\Psi_{a_{1}, a_{2}, e}^{1,1}\right)$ by

$$
\begin{aligned}
& \left(\alpha\left(\Psi_{a_{1}, a_{2}, e}^{1,1}\right)\right)\left(w_{a}, w_{a^{\prime}} ; z_{1}, z_{2} ; \tau\right)=\pi\left(\Psi_{a_{1}, a_{2}, e}^{1,1}\left(w_{a}, w_{a^{\prime}} ; z_{1}, z_{2}-1 ; \tau\right)\right), \\
& \left(\beta\left(\Psi_{a_{1}, a_{2}, e}^{1,1}\right)\right)\left(w_{a}, w_{a^{\prime}} ; z_{1}, z_{2} ; \tau\right)=\pi\left(\Psi_{a_{1}, a_{2}, e}^{1,1}\left(w_{a}, w_{a^{\prime}} ; z_{1}, z_{2}+\tau ; \tau\right)\right)
\end{aligned}
$$

for $a \in \mathcal{A}, w_{a} \in W^{a}$ and $w_{a^{\prime}} \in W^{a^{\prime}}$. 
Proposition 4.3 For $a_{1}, a_{2} \in \mathcal{A}$, we have

$$
\alpha\left(\Psi_{a_{1}, a_{2}, e}^{1,1}\right)=e^{-2 \pi i h_{a_{2}}}\left(B^{(-1)}\right)^{2}\left(\mathcal{Y}_{a_{1} e ; 1}^{a_{1}} \otimes \mathcal{Y}_{a_{2}^{\prime} a_{2} ; 1}^{e} ; \mathcal{Y}_{a_{1} e ; 1}^{a_{1}} \otimes \mathcal{Y}_{a_{2}^{\prime} a_{2} ; 1}^{e}\right) \Psi_{a_{1}, a_{2}, e}^{1,1}
$$

and

$$
\begin{gathered}
\beta\left(\Psi_{a_{1}, a_{2}, e}^{1,1}\right)=e^{-2 \pi i h_{a_{2}}} \sum_{a_{3} \in \mathcal{A}} \sum_{i=1}^{N_{a_{2} a_{3}}^{a_{1} a_{3}}} \sum_{j=1}^{N_{a_{2}^{\prime} a_{1}}^{a_{3}}} F\left(\mathcal{Y}_{a_{2} e ; 1}^{a_{2}} \otimes \mathcal{Y}_{a_{1}^{\prime} a_{1} ; 1}^{e} ; \mathcal{Y}_{a_{3}^{\prime} a_{1} ; j}^{a_{2}} \otimes \mathcal{Y}_{a_{2} a_{1}^{\prime} ; i}^{a_{3}^{\prime}}\right) . \\
\cdot F\left(\mathcal{Y}_{a_{3}^{\prime} a_{1} ; j}^{a_{2}} \otimes \sigma_{123}\left(\mathcal{Y}_{a_{2} a_{1}^{\prime} ;}^{a_{3}^{\prime}}\right) ; \mathcal{Y}_{e a_{2} ; l}^{a_{2}} \otimes \mathcal{Y}_{a_{3}^{\prime} a_{3} ; k}^{e}\right) \Psi_{a_{3}, a_{2}, e}^{1,1} .
\end{gathered}
$$

Proof. Using the definitions of $\alpha, \pi$ and $\Psi_{a_{1}, a_{2}, e}^{1,1}\left(w_{a_{2}}, w_{a_{2}^{\prime}} ; z_{1}, z_{2} ; \tau\right)$, (4.10) and (3.1), we have

$$
\begin{aligned}
& \left(\alpha\left(\Psi_{a_{1}, a_{2}, e}^{1,1}\right)\right)\left(w_{a_{2}}, w_{a_{2}^{\prime}} ; z_{1}, z_{2} ; \tau\right) \\
& =\pi\left(\Psi_{a_{1}, a_{2}, e}^{1,1}\left(w_{a_{2}}, w_{a_{2}^{\prime}} ; z_{1}, z_{2}-1 ; \tau\right)\right) \\
& =\sum_{a_{3} \in \mathcal{A}} \sum_{i=1}^{N_{a_{2} a_{3}}^{a_{1} a_{3}}} \sum_{j=1}^{N_{a_{2}^{\prime} a_{1}}^{a_{3}}} \sum_{a_{4} \in \mathcal{A}} \sum_{k=1}^{N_{a_{1}}^{a_{1} a_{1}}} \sum_{l=1}^{N_{a_{2} a_{2}^{\prime}}^{a_{4}}} e^{-2 \pi i\left(h_{a_{3}}-h_{a_{1}}\right)} \text {. } \\
& \cdot F^{-1}\left(\mathcal{Y}_{e a_{1} ; 1}^{a_{1}} \otimes \mathcal{Y}_{a_{2} a_{2}^{\prime} ; 1}^{e} ; \mathcal{Y}_{a_{2} a_{3} ; i}^{a_{1}} \otimes \mathcal{Y}_{a_{2}^{\prime} a_{1} ; j}^{a_{3}}\right) \text {. } \\
& \cdot F\left(\mathcal{Y}_{a_{2} a_{3} ; i}^{a_{1}} \otimes \mathcal{Y}_{a_{2}^{\prime} a_{1} ; j}^{a_{3}} ; \mathcal{Y}_{a_{4} a_{1} ; k}^{a_{1}} \otimes \mathcal{Y}_{a_{2} a_{2}^{\prime} ;}^{a_{4}}\right) \text {. } \\
& \cdot \pi\left(E \left(\operatorname { T r } _ { W ^ { a _ { 1 } } } \mathcal { Y } _ { a _ { 4 } a _ { 1 } ; k } ^ { a _ { 1 } } \left(\mathcal{U}\left(e^{2 \pi i z_{2}}\right) .\right.\right.\right. \\
& \left.\left.\left.\mathcal{Y}_{a_{2} a_{2}^{\prime} ; l}^{a_{4}}\left(w_{a_{2}}, z_{1}-z_{2}\right) w_{a_{2}^{\prime}}, e^{2 \pi i z_{2}}\right) q_{\tau}^{L(0)-\frac{c}{24}}\right)\right) \\
& =\sum_{a_{3} \in \mathcal{A}} \sum_{i=1}^{N_{a_{2} a_{3}}^{a_{1}}} \sum_{j=1}^{N_{a_{2}^{\prime} a_{1}}^{a_{3}}} e^{-2 \pi i\left(h_{a_{3}}-h_{a_{1}}\right)} F^{-1}\left(\mathcal{Y}_{e a_{1} ; 1}^{a_{1}} \otimes \mathcal{Y}_{a_{2} a_{2}^{\prime} ;}^{e} ; \mathcal{Y}_{a_{2} a_{3} ; i}^{a_{1}} \otimes \mathcal{Y}_{a_{2}^{\prime} a_{1} ; j}^{a_{3}}\right) \text {. } \\
& \cdot F\left(\mathcal{Y}_{a_{2} a_{3} ; i}^{a_{1}} \otimes \mathcal{Y}_{a_{2}^{\prime} a_{1} ; j}^{a_{3}} ; \mathcal{Y}_{e a_{1} ; 1}^{a_{1}} \otimes \mathcal{Y}_{a_{2} a_{2}^{\prime} ; 1}^{e}\right) . \\
& \cdot E\left(\operatorname { T r } _ { W ^ { a _ { 1 } } } \mathcal { Y } _ { \text { ea } ; 1 1 } ^ { a _ { 1 } } \left(\mathcal{U}\left(e^{2 \pi i z_{2}}\right)\right.\right. \text {. } \\
& \left.\left.\mathcal{Y}_{a_{2} a_{2}^{\prime} ; 1}^{e}\left(w_{a_{2}}, z_{1}-z_{2}\right) w_{a_{2}^{\prime}}, e^{2 \pi i z_{2}}\right) q_{\tau}^{L(0)-\frac{c}{24}}\right)
\end{aligned}
$$

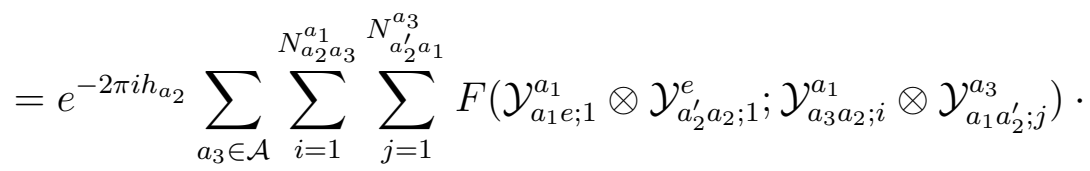




$$
\begin{gathered}
\cdot e^{-2 \pi i\left(h_{a_{3}}-h_{a_{1}}-h_{a_{2}}\right)} F^{-1}\left(\mathcal{Y}_{a_{3} a_{2} ; i}^{a_{1}} \otimes \mathcal{Y}_{a_{1} a_{2}^{\prime} ; j}^{a_{3}} ; \mathcal{Y}_{a_{1} e ; 1}^{a_{1}} \otimes \mathcal{Y}_{a_{2}^{\prime} a_{2} ; 1}^{e}\right) \cdot \\
\left.\cdot E\left(\operatorname{Tr}_{W^{a_{1}}{ } \mathcal{Y}_{e a_{1} ; 1}^{a_{1}}\left(\mathcal{U}\left(e^{2 \pi i z_{2}}\right) \cdot\right.} \cdot \mathcal{Y}_{a_{2} a_{2}^{\prime} ; 1}^{e}\left(w_{a_{2}}, z_{1}-z_{2}\right) w_{a_{2}^{\prime}}, e^{2 \pi i z_{2}}\right) q_{\tau}^{L(0)-\frac{c}{24}}\right) \\
=e^{-2 \pi i h_{a_{2}}}\left(B^{(-1)}\right)^{2}\left(\mathcal{Y}_{a_{1} e ; 1}^{a_{1}} \otimes \mathcal{Y}_{a_{2}^{\prime} a_{2} ; 1}^{e} ; \mathcal{Y}_{a_{1} e ; 1}^{a_{1}} \otimes \mathcal{Y}_{a_{2}^{\prime} a_{2} ; 1}^{e}\right) \cdot \\
\cdot \Psi_{a_{1}, a_{2}, e}^{1,1}\left(w_{a_{2}}, w_{a_{2}^{\prime}} ; z_{1}, z_{2} ; \tau\right),
\end{gathered}
$$

proving (4.18). have

Using the definitions of $\beta, \pi$ and $\Psi_{a_{1}, a_{2}, e}^{1,1}\left(w_{a_{2}}, w_{a_{2}^{\prime}} ; z_{1}, z_{2} ; \tau\right)$, (4.11), we

$$
\begin{aligned}
& \left(\beta\left(\Psi_{a_{1}, a_{2}, e}^{1,1}\right)\right)\left(w_{a_{2}}, w_{a_{2}^{\prime}} ; z_{1}, z_{2} ; \tau\right) \\
& =\pi\left(\Psi_{a_{1}, a_{2}, e}^{1,1}\left(w_{a_{2}}, w_{a_{2}^{\prime}} ; z_{1}, z_{2}+\tau ; \tau\right)\right) \\
& =\sum_{a_{3} \in \mathcal{A}} \sum_{i=1}^{N_{a_{2} a_{3}}^{a_{1} a_{3}}} \sum_{j=1}^{N_{a_{2}^{\prime} a_{1}}^{a_{3}}} \sum_{a_{4} \in \mathcal{A}} \sum_{k=1}^{N_{a_{4} a_{3}}^{a_{3}}} \sum_{l=1}^{N_{a_{2} a_{2}^{\prime}}^{a_{4}}} e^{\pi i\left(-2 h_{a_{2}}+h_{a_{4}}\right)} \\
& \cdot F^{-1}\left(\mathcal{Y}_{e a_{1} ; 1}^{a_{1}} \otimes \mathcal{Y}_{a_{2} a_{2}^{\prime} ; 1}^{e} ; \sigma_{23}\left(\mathcal{Y}_{a_{2} a_{1}^{\prime} ; i}^{a^{\prime}}\right) \otimes \sigma_{13}\left(\mathcal{Y}_{a_{3}^{\prime} a_{1} ; j}^{a_{2}}\right)\right) \cdot \\
& \cdot F\left(\mathcal{Y}_{a_{2} a_{1}^{\prime} ; i}^{a_{1}^{\prime}} \otimes \sigma_{123}\left(\mathcal{Y}_{a_{3}^{\prime} a_{1} ; j}^{a_{2}}\right) ; \mathcal{Y}_{a_{4} a_{3}^{\prime} ; k}^{a_{1}^{\prime}} \otimes \mathcal{Y}_{a_{2} a_{2}^{\prime} ; l}^{a_{4}}\right) \cdot \\
& \cdot \pi\left(E\left(\operatorname{Tr}_{W^{a_{3}}} \mathcal{Y}_{a_{4} a_{3} ; k}^{a_{3}}\left(\mathcal{U}\left(e^{2 \pi i z_{2}}\right) \mathcal{Y}_{a_{2} a_{2}^{\prime} ; l}^{a_{4}}\left(w_{a_{2}}, z_{1}-z_{2}\right) w_{a_{2}^{\prime}}, e^{2 \pi i z_{2}}\right) q_{\tau}^{L(0)-\frac{c}{24}}\right)\right) \\
& =e^{-2 \pi i h_{a_{2}}} \sum_{a_{3} \in \mathcal{A}} \sum_{i=1}^{N_{a_{2} a_{3}}^{a_{1}}} \sum_{j=1}^{N_{a_{2}^{\prime} a_{1}}^{a_{3}}} F^{-1}\left(\mathcal{Y}_{e a_{1} ; 1}^{a_{1}} \otimes \mathcal{Y}_{a_{2} a_{2}^{\prime} ; 1}^{e} ; \sigma_{23}\left(\mathcal{Y}_{a_{2} a_{1}^{\prime} ; i}^{a_{3}^{\prime}}\right) \otimes \sigma_{13}\left(\mathcal{Y}_{a_{3}^{\prime} a_{1} ; j}^{a_{2}}\right)\right) \text {. } \\
& \cdot F\left(\mathcal{Y}_{a_{2} a_{1}^{\prime} ; i}^{a_{3}^{\prime}} \otimes \sigma_{123}\left(\mathcal{Y}_{a_{3}^{\prime} a_{1} ; j}^{a_{2}}\right) ; \mathcal{Y}_{e a_{3}^{\prime} ; 1}^{a_{3}^{\prime}} \otimes \mathcal{Y}_{a_{2} a_{2}^{\prime} ; 1}^{e}\right) \text {. } \\
& \cdot E\left(\operatorname{Tr}_{W^{a_{3}}} \mathcal{Y}_{e a_{3} ; 1}^{a_{3}}\left(\mathcal{U}\left(e^{2 \pi i z_{2}}\right) \mathcal{Y}_{a_{2} a_{2}^{\prime} ; 1}^{e}\left(w_{a_{2}}, z_{1}-z_{2}\right) w_{a_{2}^{\prime}}, e^{2 \pi i z_{2}}\right) q_{\tau}^{L(0)-\frac{c}{24}}\right) \\
& =e^{-2 \pi i h_{a_{2}}} \sum_{a_{3} \in \mathcal{A}} \sum_{i=1}^{N_{a_{2} a_{3}}^{a_{1}}} \sum_{j=1}^{N_{a_{2}^{\prime} a_{1}}^{a_{3}}} F^{-1}\left(\mathcal{Y}_{e a_{1} ; 1}^{a_{1}} \otimes \mathcal{Y}_{a_{2} a_{2}^{\prime} ; 1}^{e} ; \sigma_{23}\left(\mathcal{Y}_{a_{2} a_{1}^{\prime} ; i}^{a_{3}^{\prime}}\right) \otimes \sigma_{13}\left(\mathcal{Y}_{a_{3}^{\prime} a_{1} ; j}^{a_{2}}\right)\right) \text {. } \\
& \cdot F\left(\mathcal{Y}_{a_{2} a_{1}^{\prime} ; i}^{a_{3}^{\prime}} \otimes \sigma_{123}\left(\mathcal{Y}_{a_{3}^{\prime} a_{1} ; j}^{a_{2}}\right) ; \mathcal{Y}_{e a_{3}^{\prime} ; k}^{a_{3}^{\prime}} \otimes \mathcal{Y}_{a_{2} a_{2}^{\prime} ; l}^{e}\right) \cdot \\
& \text { - } \Psi_{a_{3}, a_{2}, e}^{1,1}\left(w_{a_{2}}, w_{a_{2}^{\prime}} ; z_{1}, z_{2} ; \tau\right) .
\end{aligned}
$$

Thus we obtain

$\beta\left(\Psi_{a_{1}, a_{2}, e}^{1,1}\right)$ 


$$
\begin{aligned}
=e^{-2 \pi i h_{a_{2}}} \sum_{a_{3} \in \mathcal{A}} \sum_{i=1}^{N_{a_{2} a_{3}}^{a_{1}}} \sum_{j=1}^{N_{a_{2}^{\prime} a_{1}}^{a_{3}}} F^{-1}\left(\mathcal{Y}_{e a_{1} ; 1}^{a_{1}} \otimes \mathcal{Y}_{a_{2} a_{2}^{\prime} ; 1}^{e} ; \sigma_{23}\left(\mathcal{Y}_{a_{2} a_{1}^{\prime} ; i}^{a_{3}^{\prime}}\right) \otimes \sigma_{13}\left(\mathcal{Y}_{a_{3}^{\prime} a_{1} ; j}^{a_{2}}\right)\right) . \\
\cdot F\left(\mathcal{Y}_{a_{2} a_{1}^{\prime} ; i}^{a_{\prime}^{\prime}} \otimes \sigma_{123}\left(\mathcal{Y}_{a_{3}^{\prime} a_{1} ; j}^{a_{2}}\right) ; \mathcal{Y}_{e a_{3}^{\prime} ; k}^{a_{3}^{\prime}} \otimes \mathcal{Y}_{a_{2} a_{2}^{\prime} ; l}^{e}\right) \Psi_{a_{3}, a_{2}, e}^{1,1}
\end{aligned}
$$

Now using (3.5), (3.12) and the relations $\sigma_{12} \sigma_{23}=\sigma_{123}, \sigma_{12} \sigma_{13}=\sigma_{132}$ and $\sigma_{123} \sigma_{132}=\sigma_{132} \sigma_{123}=1$, we have

$$
\begin{aligned}
F^{-1} & \left(\mathcal{Y}_{e a_{1} ; 1}^{a_{1}} \otimes \mathcal{Y}_{a_{2} a_{2}^{\prime} ; 1}^{e} ; \sigma_{23}\left(\mathcal{Y}_{a_{2} a_{1}^{\prime} ; i}^{a_{3}^{\prime}}\right) \otimes \sigma_{13}\left(\mathcal{Y}_{a_{3}^{\prime} a_{1} ; j}^{a_{2}}\right)\right) \\
& =F\left(\sigma_{12}\left(\mathcal{Y}_{e a_{1} ; 1}^{a_{1}}\right) \otimes \sigma_{12}\left(\mathcal{Y}_{a_{2} a_{2}^{\prime} ; 1}^{e}\right) ; \sigma_{12}\left(\sigma_{23}\left(\mathcal{Y}_{a_{2} a_{1}^{\prime} ; i}^{a_{3}^{\prime}}\right)\right) \otimes \sigma_{12}\left(\sigma_{13}\left(\mathcal{Y}_{a_{3}^{\prime} a_{1} ; j}^{a_{2}}\right)\right)\right) \\
& =F\left(\mathcal{Y}_{a_{1} e ; 1}^{a_{1}} \otimes \mathcal{Y}_{a_{2}^{\prime} a_{2} ; 1}^{e} ; \sigma_{12}\left(\sigma_{23}\left(\mathcal{Y}_{a_{2} a_{1}^{\prime} ; i}^{a_{1}^{\prime}}\right)\right) \otimes \sigma_{12}\left(\sigma_{13}\left(\mathcal{Y}_{a_{3}^{\prime} a_{1} ; j}^{a_{2}}\right)\right)\right) \\
& =F\left(\mathcal{Y}_{a_{2} e ; 1}^{a_{2}} \otimes \mathcal{Y}_{a_{1}^{\prime} a_{1} ; 1}^{e} ; \sigma_{123}\left(\sigma_{132}\left(\mathcal{Y}_{a_{3}^{\prime} a_{1} ; j}^{a_{2}}\right)\right) \otimes \sigma_{132}\left(\sigma_{123}\left(\mathcal{Y}_{a_{2} a_{1}^{\prime} ; i}^{a_{3}^{\prime}}\right)\right)\right) \\
& =F\left(\mathcal{Y}_{a_{2} e ; 1}^{a_{2}} \otimes \mathcal{Y}_{a_{1}^{\prime} a_{1} ; 1}^{e} ; \mathcal{Y}_{a_{3}^{\prime} a_{1} ; j}^{a_{2}} \otimes \mathcal{Y}_{a_{2} a_{1}^{\prime} ; i}^{a_{3}^{\prime}}\right)
\end{aligned}
$$

and

$$
\begin{aligned}
& F\left(\mathcal{Y}_{a_{2} a_{1}^{\prime} ; i}^{a_{3}^{\prime}} \otimes \sigma_{123}\left(\mathcal{Y}_{a_{3}^{\prime} a_{1} ; j}^{a_{2}}\right) ; \mathcal{Y}_{e a_{3}^{\prime} ; k}^{a_{3}^{\prime}} \otimes \mathcal{Y}_{a_{2} a_{2}^{\prime} ; l}^{e}\right) \\
& \quad=F\left(\sigma_{132}\left(\sigma_{123}\left(\mathcal{Y}_{a_{3}^{\prime} a_{1} ; j}^{a_{2}}\right)\right) \otimes \sigma_{123}\left(\mathcal{Y}_{a_{2} a_{1}^{\prime} ;}^{a_{3}^{\prime}}\right) ; \sigma_{123}\left(\mathcal{Y}_{a_{2} a_{2}^{\prime} ; l}^{e}\right) \otimes \sigma_{132}\left(\mathcal{Y}_{e a_{3}^{\prime} ; k}^{a_{3}^{\prime}}\right)\right) \\
& \quad=F\left(\mathcal{Y}_{a_{3}^{\prime} a_{1} ; j}^{a_{2}} \otimes \sigma_{123}\left(\mathcal{Y}_{a_{2} a_{1}^{\prime} ; i}^{a_{3}^{\prime}}\right) ; \mathcal{Y}_{e a_{2} ; l}^{a_{2}} \otimes \mathcal{Y}_{a_{3}^{\prime} a_{3} ; k}^{e}\right) .
\end{aligned}
$$

From (4.20)-(4.22), we obtain (4.19).

By Proposition 2.11, $\Psi_{a_{1}, a_{2}, e}^{1,1}, a_{1} \in \mathcal{A}$ form a basis of $\mathcal{F}_{1 ; 2}^{e}$. For fixed $a_{2} \in \mathcal{A}$, we use $\alpha_{a_{1}}^{a_{3}}\left(a_{2}\right)$ and $\beta_{a_{1}}^{a_{3}}\left(a_{2}\right), a_{1}, a_{3} \in \mathcal{A}$, to denote the matrix elements of $\alpha$ and $\beta$, respectively, under the basis $\Psi_{a_{1}, a_{2}, e}^{1,1}, a_{1} \in \mathcal{A}$.

Corollary 4.4 The matrix elements $\alpha_{a_{1}}^{a_{3}}\left(a_{2}\right)$ and $\beta_{a_{1}}^{a_{3}}\left(a_{2}\right), a_{1}, a_{3} \in \mathcal{A}$, are given by

$$
\alpha_{a_{1}}^{a_{3}}\left(a_{2}\right)=\delta_{a_{1} a_{3}}\left(B^{(-1)}\right)^{2}\left(\mathcal{Y}_{a_{1} e ; 1}^{a_{1}} \otimes \mathcal{Y}_{a_{2}^{\prime} a_{2} ; 1}^{e} ; \mathcal{Y}_{a_{1} e ; 1}^{a_{1}} \otimes \mathcal{Y}_{a_{2}^{\prime} a_{2} ; 1}^{e}\right)
$$

and

$$
\begin{aligned}
\beta_{a_{1}}^{a_{3}}\left(a_{2}\right)=e^{-2 \pi i h_{a_{2}}} \sum_{i=1}^{N_{a_{2} a_{3}}^{a_{1}}} \sum_{j=1}^{N_{a_{2}^{\prime} a_{1}}^{a_{3}}} F\left(\mathcal{Y}_{a_{2} e ; 1}^{a_{2}} \otimes \mathcal{Y}_{a_{1}^{\prime} a_{1} ; 1}^{e} ; \mathcal{Y}_{a_{3}^{\prime} a_{1} ; j}^{a_{2}} \otimes \mathcal{Y}_{a_{2} a_{1}^{\prime} ; i}^{a_{3}^{\prime}}\right) . \\
\cdot F\left(\mathcal{Y}_{a_{3}^{\prime} a_{1} ; j}^{a_{2}} \otimes \sigma_{123}\left(\mathcal{Y}_{a_{2} a_{1}^{\prime} ; i}^{a_{3}^{\prime}}\right) ; \mathcal{Y}_{e a_{2} ; l}^{a_{2}} \otimes \mathcal{Y}_{a_{3}^{\prime} a_{3} ; k}^{e}\right) .
\end{aligned}
$$


Proof. This corollary follows directly from the definition of $\alpha_{a_{1}}^{a_{3}}\left(a_{2}\right)$ and $\beta_{a_{1}}^{a_{3}}\left(a_{2}\right), a_{1}, a_{3} \in \mathcal{A}$, (4.18) and (4.19).

It is also easy to establish the relationship between $\alpha$ and $\beta$ :

Proposition 4.5 We have the following formula:

$$
S \alpha S^{-1}=\beta .
$$

Proof. We have

$$
\begin{aligned}
&\left(\beta\left(S\left(\Psi_{a_{1}, a_{2}, e}^{1,1}\right)\right)\right)\left(w_{a_{2}}, w_{a_{2}^{\prime}} ; z_{1}, z_{2} ; \tau\right)=\pi\left(S\left(\Psi_{a_{1}, a_{2}, e}^{1,1}\right)\left(w_{a_{2}}, w_{a_{2}^{\prime}} ; z_{1}, z_{2}+\tau ; \tau\right)\right) \\
&= \pi\left(E \left(\operatorname { T r } _ { W ^ { a _ { 1 } } \mathcal { Y } _ { e a _ { 1 } ; 1 } } \left(\mathcal{U}\left(e^{-2 \pi i \frac{z_{2}+\tau}{\tau}}\right)\left(-\frac{1}{\tau}\right)^{L(0)} \cdot\right.\right.\right. \\
&\left.\left.\left.\cdot \mathcal{Y}_{a_{2} a_{2}^{\prime} ; 1}^{e}\left(w_{a_{2}}, z_{1}-z_{2}+\tau\right) w_{a_{2}^{\prime}}, e^{-2 \pi i \frac{z_{2}+\tau}{\tau}}\right) q_{-\frac{1}{\tau}}^{L(0)-\frac{c}{24}}\right)\right) \\
&=\pi\left(E \left(\operatorname{Tr}_{W^{a_{1}} \mathcal{Y}_{e a_{1} ; 1}^{a_{1}}\left(\mathcal{U}\left(e^{2 \pi i\left(-\frac{z_{2}}{\tau}-1\right)}\right) \cdot\right.} \cdot \mathcal{Y}_{a_{2} a_{2}^{\prime} ; 1}^{e}\left(\left(-\frac{1}{\tau}\right)^{L(0)} w_{a_{2}},-\frac{1}{\tau} z_{1}-\left(-\frac{1}{\tau} z_{2}-1\right)\right) \cdot\right.\right. \\
&\left.\left.\left.\quad \cdot\left(-\frac{1}{\tau}\right)^{L(0)} w_{a_{2}^{\prime}}, e^{2 \pi i\left(-\frac{z_{2}}{\tau}-1\right)}\right)^{L(0)-\frac{c}{24}}\right)\right) \\
&=\pi\left(\Psi_{a_{1}, a_{2}, e}^{1,1}\left(\left(-\frac{1}{\tau}\right)^{L(0)} w_{a_{2}},\left(-\frac{1}{\tau}\right)^{L(0)} w_{a_{2}^{\prime}} ;-\frac{1}{\tau} z_{1},-\frac{1}{\tau} z_{2}-1 ;-\frac{1}{\tau}\right)\right) \\
&=\left(\alpha\left(\Psi_{a_{1}, a_{2}, e}^{1,1}\right)\right)\left(\left(-\frac{1}{\tau}\right)^{L(0)} w_{a_{2}},\left(-\frac{1}{\tau}\right)^{L(0)} w_{a_{2}^{\prime}} ;-\frac{1}{\tau} z_{1},-\frac{1}{\tau} z_{2} ;-\frac{1}{\tau}\right) \\
&=\left(S\left(\alpha\left(\Psi_{a_{1}, a_{2}, e}^{1,1}\right)\right)\left(w_{a_{2}}, w_{a_{2}^{\prime}} ; z_{1}, z_{2} ; \tau\right) .\right.
\end{aligned}
$$

Thus we obtain

$$
\beta S=S \alpha
$$

or equivalently (4.25).

With the bases of the spaces of intertwining operators we choose in the beginning of this section, we have

$$
\Psi_{a_{1}}(u ; \tau)=\operatorname{Tr}_{W^{a_{1}}} \mathcal{Y}_{e a_{1} ; 1}^{a_{1}}\left(\mathcal{U}\left(e^{2 \pi i z}\right) u, e^{2 \pi i z}\right) q_{\tau}^{L(0)-\frac{c}{24}}
$$




$$
=\operatorname{Tr}_{W^{a_{1}}} Y_{W^{a_{1}}}\left(\mathcal{U}\left(e^{2 \pi i z}\right) u, e^{2 \pi i z}\right) q_{\tau}^{L(0)-\frac{c}{24}}
$$

for $a_{1} \in \mathcal{A}, u \in V$ and

$$
\begin{aligned}
& \Psi_{a_{1}, a_{2}, e}^{1,1}\left(w_{a_{2}}, w_{a_{2}^{\prime}} ; z_{1}, z_{2} ; \tau\right) \\
& \quad=E\left(\operatorname{Tr}_{W^{a_{1}}} \mathcal{Y}_{e a_{1} ; 1}^{a_{1}}\left(\mathcal{U}\left(e^{2 \pi i z_{2}}\right) \mathcal{Y}_{a_{2} a_{2}^{\prime} ; 1}^{e}\left(w_{a_{2}}, z_{1}-z_{2}\right) w_{a_{2}^{\prime}}, e^{2 \pi i z_{2}}\right) q_{\tau}^{L(0)-\frac{c}{24}}\right) \\
& \quad=E\left(\operatorname{Tr}_{W^{a_{1}}} Y_{W^{a_{1}}}\left(\mathcal{U}\left(e^{2 \pi i z_{2}}\right) \mathcal{Y}_{a_{2} a_{2}^{\prime} ; 1}^{e}\left(w_{a_{2}}, z_{1}-z_{2}\right) w_{a_{2}^{\prime}}, e^{2 \pi i z_{2}}\right) q_{\tau}^{L(0)-\frac{c}{24}}\right)
\end{aligned}
$$

for $a_{1}, a_{2} \in \mathcal{A}, w_{a_{2}} \in W^{a_{2}}$ and $w_{a_{2}^{\prime}} \in W^{a_{2}^{\prime}}$.

Since $\Psi_{a_{1}}$ for $a_{1} \in \mathcal{A}$ are linear independent, they form a basis of $\mathcal{F}_{1 ; 1}^{e}$. Thus we know that there exist unique $S_{a_{1}}^{a_{3}} \in \mathbb{C}$ for $a_{1}, a_{3} \in \mathcal{A}$ such that

$$
\begin{aligned}
\left(S\left(\Psi_{a_{1}}\right)\right)(u ; \tau) & =\sum_{a_{3} \in \mathcal{A}} S_{a_{3}}^{a_{1}} \Psi_{a_{3}}(u ; \tau), \\
S\left(\Psi_{a_{1}}\right) & =\sum_{a_{3} \in \mathcal{A}} S_{a_{3}}^{a_{1}} \Psi_{a_{3}} .
\end{aligned}
$$

Clearly we also have

$$
S\left(\Psi_{a_{1}, a_{2}, e}^{1,1}\right)=\sum_{a_{3} \in \mathcal{A}} S_{a_{3}}^{a_{1}} \Psi_{a_{3}, a_{2}, e}^{1,1}
$$

for $a_{1}, a_{2} \in \mathcal{A}$.

We can identify the space spanned by $\Psi_{a}$ for $a \in \mathcal{A}$ with the vector space $\coprod_{a \in \mathcal{A}} \mathbb{C}\left[W^{a}\right]$ where $\left[W^{a}\right]$ is the equivalence class of $W^{a}$ and is actually equal to $a$. Then we can view $S$ as a linear operator on this vector space spanned by $\mathcal{A}$. In terms of the basis $\left[W^{a}\right], a \in \mathcal{A}$, we have

$$
S\left(\left[W^{a_{1}}\right]\right)=\sum_{a_{2} \in \mathcal{A}} S_{a_{2}}^{a_{1}}\left[W^{a_{2}}\right]
$$

Let $u=\mathbf{1}$ in (4.26). Then we see that $S_{a_{1}}^{a_{2}}, a_{1}, a_{2} \in \mathcal{A}$, gives an action of the modular transformation $\tau \mapsto-1 / \tau$ on the space spanned by shifted graded dimensions (vacuum characters) of irreducible $V$-modules.

The following theorem gives the second Moore-Seiberg formula in [MS1]:

Theorem 4.6 For $a_{1}, a_{2}, a_{3} \in \mathcal{A}$, we have

$$
\sum_{a_{4} \in \mathcal{A}} S_{a_{1}}^{a_{4}}\left(B^{(-1)}\right)^{2}\left(\mathcal{Y}_{a_{4} e ; 1}^{a_{4}} \otimes \mathcal{Y}_{a_{2}^{\prime} a_{2} ; 1}^{e} ; \mathcal{Y}_{a_{4} e ; 1}^{a_{4}} \otimes \mathcal{Y}_{a_{2}^{\prime} a_{2} ; 1}^{e}\right)\left(S^{-1}\right)_{a_{4}}^{a_{3}}
$$




$$
\begin{aligned}
=\sum_{i=1}^{N_{a_{1} a_{2}}^{a_{3}}} \sum_{k=1}^{N_{a_{1}^{\prime} a_{3}}^{a_{2}}} F\left(\mathcal{Y}_{a_{2} e ; 1}^{a_{2}} \otimes \mathcal{Y}_{a_{3}^{\prime} a_{3} ; 1}^{e} ; \mathcal{Y}_{a_{1}^{\prime} a_{3} ; k}^{a_{2}} \otimes \mathcal{Y}_{a_{2} a_{3}^{\prime} ; i}^{a_{1}^{\prime}}\right) \\
\cdot F\left(\mathcal{Y}_{a_{1}^{\prime} a_{3} ; k}^{a_{2}} \otimes \sigma_{123}\left(\mathcal{Y}_{a_{2} a_{3}^{\prime} ; i}^{a_{1}^{\prime}}\right) ; \mathcal{Y}_{e a_{2} ; 1}^{a_{2}} \otimes \mathcal{Y}_{a_{1}^{\prime} a_{1} ; 1}^{e}\right)
\end{aligned}
$$

Proof. This follows from (4.25), (4.23) and (4.24) immediately.

Corollary 4.7 For $a_{1}, a_{2}, a_{3} \in \mathcal{A}$, we have

$$
\begin{gathered}
\sum_{a_{4} \in \mathcal{A}} S_{a_{1}}^{a_{4}}\left(B^{(-1)}\right)^{2}\left(\mathcal{Y}_{a_{4} e ; 1}^{a_{4}} \otimes \mathcal{Y}_{a_{2}^{\prime} a_{2} ; 1}^{e} ; \mathcal{Y}_{a_{4} e ; 1}^{a_{4}} \otimes \mathcal{Y}_{a_{2}^{\prime} a_{2} ; 1}^{e}\right)\left(S^{-1}\right)_{a_{4}}^{a_{3}} \\
=N_{a_{1} a_{2}}^{a_{3}} F\left(\mathcal{Y}_{a_{2} e ; 1}^{a_{2}} \otimes \mathcal{Y}_{a_{2}^{\prime} a_{2} ; 1}^{e} ; \mathcal{Y}_{e a_{2} ; 1}^{a_{2}} \otimes \mathcal{Y}_{a_{2} a_{2}^{\prime} ; 1}^{e}\right) .
\end{gathered}
$$

Proof. This follows immediately from (4.9) and (4.28).

\section{The main theorem and the Verlinde for- mula}

In this section, using the results obtained in the preceding section, we prove the main theorem, the Verlinde conjecture, of the present paper, derive the Verlinde formula for fusion rules and prove that $\left(S_{a_{1}}^{a_{2}}\right)$ is symmetric.

First, we have the following:

Proposition 5.1 For $a_{2} \in \mathcal{A}$,

$$
F\left(\mathcal{Y}_{a_{2} e ; 1}^{a_{2}} \otimes \mathcal{Y}_{a_{2}^{\prime} a_{2} ; 1}^{e} ; \mathcal{Y}_{e a_{2} ; 1}^{a_{2}} \otimes \mathcal{Y}_{a_{2} a_{2}^{\prime} ; 1}^{e}\right) \neq 0
$$

Proof. If

$$
F\left(\mathcal{Y}_{a_{2} e ; 1}^{a_{2}} \otimes \mathcal{Y}_{a_{2}^{\prime} a_{2} ; 1}^{e} ; \mathcal{Y}_{e a_{2} ; 1}^{a_{2}} \otimes \mathcal{Y}_{a_{2} a_{2}^{\prime} ; 1}^{e}\right)=0
$$

then by (4.29),

$$
\left(B^{(-1)}\right)^{2}\left(\mathcal{Y}_{a_{4} e ; 1}^{a_{4}} \otimes \mathcal{Y}_{a_{2}^{\prime} a_{2} ; 1}^{e} ; \mathcal{Y}_{a_{4} e ; 1}^{a_{4}} \otimes \mathcal{Y}_{a_{2}^{\prime} a_{2} ; 1}^{e}\right)=0
$$

for $a_{4} \in \mathcal{A}$. But we know that

$$
\left(B^{(-1)}\right)^{2}\left(\mathcal{Y}_{e e ; 1}^{e} \otimes \mathcal{Y}_{a_{2}^{\prime} a_{2} ; 1}^{e} ; \mathcal{Y}_{e e ; 1}^{e} \otimes \mathcal{Y}_{a_{2}^{\prime} a_{2} ; 1}^{e}\right)=1
$$


Contradiction.

For $a_{2} \in \mathcal{A}$, let $\mathbb{N}\left(a_{2}\right)$ be the matrices whose entries are $N_{a_{1} a_{2}}^{a_{3}}=N_{a_{2} a_{1}}^{a_{3}}$ for $a_{1}, a_{3} \in \mathcal{A}$, that is,

$$
\mathcal{N}\left(a_{2}\right)=\left(N_{a_{1} a_{2}}^{a_{3}}\right)=\left(N_{a_{2} a_{1}}^{a_{3}}\right) .
$$

Then we have the main result of the present paper:

Theorem 5.2 Let $V$ be a simple vertex operator algebra satisfying the conditions in Section 1. Then we have

$$
\sum_{a_{1}, a_{3} \in \mathcal{A}}\left(S^{-1}\right)_{a_{4}}^{a_{1}} N_{a_{1} a_{2}}^{a_{3}} S_{a_{3}}^{a_{5}}=\delta_{a_{4}}^{a_{5}} \frac{\left(B^{(-1)}\right)^{2}\left(\mathcal{Y}_{a_{4} e ; 1}^{a_{4}} \otimes \mathcal{Y}_{a_{2}^{\prime} a_{2} ; 1}^{e} ; \mathcal{Y}_{a_{4} e ; 1}^{a_{4}} \otimes \mathcal{Y}_{a_{2}^{\prime} a_{2} ; 1}^{e}\right)}{F\left(\mathcal{Y}_{a_{2} e ; 1}^{a_{2}} \otimes \mathcal{Y}_{a_{2}^{\prime} a_{2} ; 1}^{e} ; \mathcal{Y}_{e a_{2} ; 1}^{a_{2}} \otimes \mathcal{Y}_{a_{2} a_{2}^{\prime} ; 1}^{e}\right)}
$$

In particular, the matrix $S$ diagonalizes the matrices $\mathcal{N}\left(a_{2}\right)$ for all $a_{2} \in \mathcal{A}$.

Proof. By Proposition 5.1, we can rewrite (4.29) as (5.1). Since the righthand side of (5.1) are entries of diagonal matrices, $S$ diagonalizes the $\mathcal{N}\left(a_{2}\right)$ for all $a_{2} \in \mathcal{A}$.

We now prove the Verlinde formula for fusion rules. We first need the following:

Proposition 5.3 The square $S^{2}$ viewed as a linear operator on the vector space spanned by $\mathcal{A}$ is equal to the linear operator obtained from the map ' $: \mathcal{A} \rightarrow \mathcal{A}$.

Proof. By definition, we have

$$
\begin{aligned}
\left(S^{2}\left(\Psi_{a}\right)\right)(u ; \tau) & =\left(S\left(\Psi_{a}\right)\left(\left(-\frac{1}{\tau}\right)^{L(0)} u ;-\frac{1}{\tau}\right)\right. \\
& =\Psi_{a}\left(\left(-\frac{1}{-\frac{1}{\tau}}\right)^{L(0)}\left(-\frac{1}{\tau}\right)^{L(0)} u ;-\frac{1}{-\frac{1}{\tau}}\right) \\
& =\Psi_{a}\left(\tau^{L(0)}\left(-\frac{1}{\tau}\right)^{L(0)} u ; \tau\right) \\
& =\Psi_{a}\left(e^{\left(\log \tau+\log \left(-\frac{1}{\tau}\right)\right) L(0)} u ; \tau\right) .
\end{aligned}
$$


Note that both $\tau$ and $-\frac{1}{\tau}$ are in the upper half plane. So $0<\arg \tau, \arg \left(-\frac{1}{\tau}\right)<$ $\pi$. Thus by our convention,

$$
\arg \tau+\arg \left(-\frac{1}{\tau}\right)=\arg (-1)=\pi
$$

So we have

$$
\begin{aligned}
\log \tau+\log \left(-\frac{1}{\tau}\right) & =\log |\tau|+i \arg \tau \log \left|-\frac{1}{\tau}\right|+i \arg \left(-\frac{1}{\tau}\right) \\
& =\pi i
\end{aligned}
$$

Using (5.3) and (2.20), we see that the right-hand side of (5.2) is equal to

$$
\begin{aligned}
\Psi_{a}\left(e^{\pi i L(0)} u ; \tau\right) & =\operatorname{Tr}_{W^{a}} Y_{W^{a}}\left(\mathcal{U}\left(e^{-2 \pi i z}\right) e^{\pi i L(0)} u, e^{-2 \pi i z}\right) q_{\tau}^{L(0)-\frac{c}{24}} \\
& \left.=\operatorname{Tr}_{W^{a^{\prime}}} Y_{W^{a^{\prime}}}\left(\mathcal{U}\left(e^{2 \pi i z_{2}}\right) u, e^{2 \pi i z}\right) q_{\tau}^{L(0)-\frac{c}{24}}\right) \\
& =\Psi_{a^{\prime}}(u ; \tau)
\end{aligned}
$$

Combining (5.2) and (5.4), we obtain

$$
S^{2}\left(\Psi_{a}\right)=\Psi_{a^{\prime}}
$$

proving the conclusion.

An immediate consequence of the proposition above is the following:

Corollary 5.4 The inverse $S^{-1}$ of $S$ is equal to $S \circ^{\prime}=^{\prime} \circ S$. In particular, we have

$$
\begin{aligned}
\left(S^{-1}\right)_{a_{1}}^{a_{2}} & =S_{a_{1}}^{a_{2}^{\prime}} \\
& =S_{a_{1}^{\prime}}^{a_{2}}
\end{aligned}
$$

for $a_{1}, a_{2} \in \mathcal{A}$.

Now we have the following Verlinde formula for fusion rules:

Theorem 5.5 Let $V$ be a vertex operator algebra satisfying the conditions in Section 1. Then we have $S_{e}^{a} \neq 0$ for $a \in \mathcal{A}$ and

$$
N_{a_{1} a_{2}}^{a_{3}}=\sum_{a_{4} \in \mathcal{A}} \frac{S_{a_{1}}^{a_{4}} S_{a_{2}}^{a_{4}} S_{a_{4}}^{a_{3}^{\prime}}}{S_{e}^{a_{4}}} .
$$


Proof. Let

$$
\lambda_{a_{2}}^{a_{4}}=\frac{\left(B^{(-1)}\right)^{2}\left(\mathcal{Y}_{a_{4} e ; 1}^{a_{4}} \otimes \mathcal{Y}_{a_{2}^{\prime} a_{2} ; 1}^{e} ; \mathcal{Y}_{a_{4} e ; 1}^{a_{4}} \otimes \mathcal{Y}_{a_{2}^{\prime} a_{2} ; 1}^{e}\right)}{F\left(\mathcal{Y}_{a_{2} e ; 1}^{a_{2}} \otimes \mathcal{Y}_{a_{2}^{\prime} a_{2} ; 1}^{e} ; \mathcal{Y}_{e a_{2} ; 1}^{a_{2}} \otimes \mathcal{Y}_{a_{2} a_{2}^{\prime} ; 1}^{e}\right)}
$$

for $a_{2}, a_{4} \in \mathcal{A}$. Then by (5.1), we have

$$
\sum_{a_{1}, a_{3} \in \mathcal{A}}\left(S^{-1}\right)_{a_{4}}^{a_{1}} N_{a_{1} a_{2}}^{a_{3}} S_{a_{3}}^{a_{5}}=\delta_{a_{4}}^{a_{5}} \lambda_{a_{2}}^{a_{4}}
$$

or equivalently

$$
N_{a_{1} a_{2}}^{a_{3}}=\sum_{a_{4} \in \mathcal{A}} S_{a_{1}}^{a_{4}} \lambda_{a_{2}}^{a_{4}}\left(S^{-1}\right)_{a_{4}}^{a_{3}}
$$

Using (5.5), we see that (5.8) becomes

$$
N_{a_{1} a_{2}}^{a_{3}}=\sum_{a_{4} \in \mathcal{A}} S_{a_{1}}^{a_{4}} \lambda_{a_{2}}^{a_{4}} S_{a_{4}}^{a_{3}^{\prime}}
$$

We know that $N_{e a_{2}}^{a_{3}}=\delta_{a_{2}}^{a_{3}}$. Combining this fact with (5.8), we obtain

$$
\delta_{a_{2}}^{a_{3}}=\sum_{a_{4} \in \mathcal{A}} S_{e}^{a_{4}} \lambda_{a_{2}}^{a_{4}}\left(S^{-1}\right)_{a_{4}}^{a_{3}} .
$$

Thus we have

$$
S_{e}^{a_{4}} \lambda_{a_{2}}^{a_{4}}=S_{a_{2}}^{a_{4}} .
$$

From (5.10) we see that if $S_{e}^{a_{4}}=0$ for some $a_{4} \in \mathcal{A}$, then there is one column of the matrix $S$ is 0 . Contradictary to the fact that $S$ is invertible. So $S_{e}^{a_{4}} \neq 0$ for $a_{4} \in \mathcal{A}$. Rewrite (5.10) as

$$
\lambda_{a_{2}}^{a_{4}}=\frac{S_{a_{2}}^{a_{4}}}{S_{e}^{a_{4}}}
$$

Substituting (5.11) into (5.9), we obtain (5.6).

Finally we have:

Theorem 5.6 The matrix $\left(S_{a_{1}}^{a_{2}}\right)$ is symmetric. 
Proof. Rewriting (5.8) as

$$
\sum_{a_{1}}\left(S^{-1}\right)_{a_{4}}^{a_{1}} N_{a_{1} a_{2}}^{a_{3}}=\lambda_{a_{2}}^{a_{4}}\left(S^{-1}\right)_{a_{4}}^{a_{3}}
$$

and then letting $a_{3}=a_{4}=e$ in $(\underline{5.12})$ and using $N_{a_{1} a_{2}}^{e}=\delta_{a_{1}}^{a_{2}^{\prime}}$, we obtain

$$
\left(S^{-1}\right)_{e}^{a_{2}^{\prime}}=\lambda_{a_{2}}^{e}\left(S^{-1}\right)_{e}^{e} .
$$

Using (5.5), (5.13) can be written as

$$
S_{e}^{a_{2}}=\lambda_{a_{2}}^{e} S_{e}^{e}
$$

By (5.11), (5.14) and (5.7),

$$
\begin{aligned}
S_{a_{2}}^{a_{4}} & =\lambda_{a_{4}^{\prime}}^{e} S_{e}^{e} \lambda_{a_{2}}^{a_{4}} \\
& =\frac{S_{e}^{e}\left(\left(B^{(-1)}\right)^{2}\left(\mathcal{Y}_{a_{4} e ; 1}^{a_{4}} \otimes \mathcal{Y}_{a_{2}^{\prime} a_{2} ; 1}^{e} ; \mathcal{Y}_{a_{4} e ; 1}^{a_{4}} \otimes \mathcal{Y}_{a_{2}^{\prime} a_{2} ; 1}^{e}\right)\right)}{F\left(\mathcal{Y}_{a_{2} e ; 1}^{a_{2}} \otimes \mathcal{Y}_{a_{2}^{\prime} a_{2} ; 1}^{e} ; \mathcal{Y}_{e a_{2} ; 1}^{a_{2}} \otimes \mathcal{Y}_{a_{2} a_{2}^{\prime} ; 1}^{e}\right) F\left(\mathcal{Y}_{a_{4} e ; 1}^{a_{4}} \otimes \mathcal{Y}_{a_{4}^{\prime} a_{4} ; 1}^{e} ; \mathcal{Y}_{e a_{4} ; 1}^{a_{4}} \otimes \mathcal{Y}_{a_{4} a_{4}^{\prime} ; 1}^{e}\right)} .
\end{aligned}
$$

By (5.15), we obtain

$$
S_{a_{2}}^{a_{4}^{\prime}}=\frac{S_{e}^{e}\left(\left(B^{(-1)}\right)^{2}\left(\mathcal{Y}_{a_{4}^{\prime} e ; 1}^{a_{4}^{\prime}} \otimes \mathcal{Y}_{a_{2}^{\prime} a_{2} ; 1}^{e} ; \mathcal{Y}_{a_{4}^{\prime} e ; 1}^{a_{4}^{\prime}} \otimes \mathcal{Y}_{a_{2}^{\prime} a_{2} ; 1}^{e}\right)\right)}{F\left(\mathcal{Y}_{a_{2} e ; 1}^{a_{2}} \otimes \mathcal{Y}_{a_{2}^{\prime} a_{2} ; 1}^{e} ; \mathcal{Y}_{e a_{2} ; 1}^{a_{2}} \otimes \mathcal{Y}_{a_{2} a_{2}^{\prime} ; 1}^{e}\right) F\left(\mathcal{Y}_{a_{4}^{\prime} e ; 1}^{a_{4}^{\prime}} \otimes \mathcal{Y}_{a_{4} a_{4}^{\prime} ; 1}^{e} ; \mathcal{Y}_{e a_{4}^{\prime} ; 1}^{a_{4}^{\prime}} \otimes \mathcal{Y}_{a_{4}^{\prime} a_{4} ; 1}^{e}\right)}
$$

From (3.1), (3.12), (3.5) and the choice of the bases of the spaces of intertwining operators when some of the modules involved are $V$, we have

$$
\begin{aligned}
& \left(B^{(-1)}\right)^{2}\left(\mathcal{Y}_{a_{4}^{\prime} e ; 1}^{a_{4}^{\prime}} \otimes \mathcal{Y}_{a_{2}^{\prime} a_{2} ; 1}^{e} ; \mathcal{Y}_{a_{4}^{\prime} e ; 1}^{a_{1}^{\prime}} \otimes \mathcal{Y}_{a_{2}^{\prime} a_{2} ; 1}^{e}\right) \\
& \quad=\left(B^{(-1)}\right)^{2}\left(\mathcal{Y}_{a_{2} e ; 1}^{a_{2}} \otimes \mathcal{Y}_{a_{4} a_{4}^{\prime} ; 1}^{e} ; \mathcal{Y}_{a_{2} e ; 1}^{a_{2}} \otimes \mathcal{Y}_{a_{4} a_{4}^{\prime} ; 1}^{e}\right) .
\end{aligned}
$$

Using (5.17) and (5.15), we see that the right-hand side of (5.16) is equal to

$$
\frac{S_{e}^{e}\left(\left(B^{(-1)}\right)^{2}\left(\mathcal{Y}_{a_{2} e ; 1}^{a_{2}} \otimes \mathcal{Y}_{a_{4} a_{4}^{\prime} ; 1}^{e} ; \mathcal{Y}_{a_{2} e ; 1}^{a_{2}} \otimes \mathcal{Y}_{a_{4} a_{4}^{\prime} ; 1}^{e}\right)\right)}{F\left(\mathcal{Y}_{a_{2}^{\prime} e ; 1}^{a_{2}^{\prime}} \otimes \mathcal{Y}_{a_{2} a_{2}^{\prime} ; 1}^{e} ; \mathcal{Y}_{e a_{2}^{\prime} ; 1}^{a_{2}^{\prime}} \otimes \mathcal{Y}_{a_{2}^{\prime} a_{2} ; 1}^{e}\right) F\left(\mathcal{Y}_{a_{4} e ; 1}^{a_{4}} \otimes \mathcal{Y}_{a_{4}^{\prime} a_{4} ; 1}^{e} ; \mathcal{Y}_{e a_{4}^{\prime} ; 1}^{a^{\prime}} \otimes \mathcal{Y}_{a_{4} a_{4}^{\prime} ; 1}^{e}\right)}=S_{a_{4}^{\prime}}^{a_{2}}
$$

The formulas (5.16) and (5.18) gives

$$
S_{a_{2}}^{a_{4}^{\prime}}=S_{a_{4}^{\prime}}^{a_{2}},
$$

proving that $\left(S_{a_{1}}^{a_{2}}\right)$ is symmetric. 


\section{References}

[ABD] T. Abe, G. Buhl, C. Dong, Rationality, Regularity, and $C_{2^{-}}$ cofiniteness, Trans. Amer. Math. Soc. 356 (2004), 3391-3402.

[AN] T. Abe and K. Nagatomo, Finiteness of conformal blocks over the projective line, in: Vertex operator algebras in mathematics and physics (Toronto, 2000), ed. S. Berman, Y. Billig, Y.-Z. Huang and J. Lepowsky, Fields Inst. Commun., Vol. 39, Amer. Math. Soc., Providence, 2003, 1-12.

[AM] G. Anderson and G. Moore, Rationality in conformal field theory, Comm. Math. Phys.117 (1988), 441-450.

[BL] A. Beauville and Y. Laszlo, Conformal blocks and generalized theta functions, Comm. Math. Phys. 164 (1994), 385-419.

[B] R. E. Borcherds, Vertex algebras, Kac-Moody algebras, and the Monster, Proc. Natl. Acad. Sci. USA 83 (1986), 3068-3071.

[DLM] C. Dong, H. Li and G. Mason, Modular-invariance of trace functions in orbifold theory and generalized Moonshine, Comm. Math. Phys. 214 (2000), 1-56.

[F] G. Faltings, A proof for the Verlinde formula, J. Alg. Geom. 3 (1994), 347-374.

[FHL] I. B. Frenkel, Y.-Z. Huang and J. Lepowsky, On axiomatic approaches to vertex operator algebras and modules, preprint, 1989; Memoirs Amer. Math. Soc. 104, 1993.

[FLM] I. B. Frenkel, J. Lepowsky, and A. Meurman, Vertex operator algebras and the Monster, Pure and Appl. Math., Vol. 134, Academic Press, New York, 1988.

[GN] M. R. Gaberdiel and A. Neitzke, Rationality, quasirationality and finite W-algebras, Comm. Math. Phys. 238 (2003), 305-331.

[H1] Y.-Z. Huang, A theory of tensor products for module categories for a vertex operator algebra, IV, J. Pure Appl. Alg. 100 (1995), 173-216. 
[H2] Y.-Z. Huang, Virasoro vertex operator algebras, (nonmeromorphic) operator product expansion and the tensor product theory, J. Alg. 182 (1996), 201-234.

[H3] Y.-Z. Huang, Two-dimensional conformal geometry and vertex operator algebras, Progress in Mathematics, Vol. 148, 1997, Birkhäuser, Boston.

[H4] Y.-Z. Huang, Intertwining operator algebras, genus-zero modular functors and genus-zero conformal field theories, in: Operads: Proceedings of Renaissance Conferences, ed. J.-L. Loday, J. Stasheff, and A. A. Voronov, Contemporary Math., Vol. 202, Amer. Math. Soc., Providence, 1997, 335-355.

[H5] Y.-Z. Huang, Generalized rationality and a "Jacobi identity" for intertwining operator algebras, Selecta Math. (N. S.), 6 (2000), 225-267.

[H6] Y.-Z. Huang, Differential equations and intertwining operators, Comm. Contemp. Math. 7 (2005), 375-400.

[H7] Y.-Z. Huang, Differential equations, duality and modular invariance, Comm. Contemp. Math. 7 (2005), 649-706.

[H8] Y.-Z. Huang, Vertex operator algebras, the Verlinde conjecture and modular tensor categories, Proc. Natl. Acad. Sci. USA 102 (2005), $5352-5356$.

[H9] Y.-Z. Huang, Vertex operator algebras, fusion rules and modular transformations, in: Non-commutative Geometry and Representation Theory in Mathematical Physics, ed. J. Fuchs, J. Mickelsson, G. Rozenblioum and A. Stolin, Contemporary Math. Vol. 391, Amer. Math. Soc., Providence, 2005, 135-148.

[H10] Y.-Z. Huang, Rigidity and modularity of vertex tensor categories, Comm. Contemp. Math., to appear; math.QA/0502533.

[HK1] Y.-Z. Huang and L. Kong, Full field algebras, Comm. Math. Phys. 272 (2007), 345-396. 
[HK2] Y.-Z. Huang and L. Kong, Modular invariance for conformal full field algebras, Amer. J. Math., to appear.

[HL1] Y.-Z. Huang and J. Lepowsky, A theory of tensor products for module categories for a vertex operator algebra, I, Selecta Math. (N.S.) 1 (1995), 699-756.

[HL2] Y.-Z. Huang and J. Lepowsky, A theory of tensor products for module categories for a vertex operator algebra, II, Selecta Math. (N.S.) 1 (1995), 757-786.

[HL3] Y.-Z. Huang and J. Lepowsky, Tensor products of modules for a vertex operator algebra and vertex tensor categories, in: Lie Theory and Geometry, in honor of Bertram Kostant, ed. R. Brylinski, J.-L. Brylinski, V. Guillemin, V. Kac, Birkhäuser, Boston, 1994, 349383.

[HL4] Y.-Z. Huang and J. Lepowsky, A theory of tensor products for module categories for a vertex operator algebra, III, J. Pure Appl. Alg. 100 (1995), 141-171.

[K] M. Kontsevich, Rational conformal field theory and invariants of 3-dimensional manifolds, preprint CPT-88/P.2189, University of Marseille, 1988.

[KNR] S. Kumar, M. S. Narasimhan and A. Ramanathan, Infinite Grassmannians and moduli spaces of G-bundles, Math. Ann. 300 (1994), $41-75$.

[Le] J. Lepowsky, From the representation theory of vertex operator algebras to modular tensor categories in conformal field theory, Proc. Natl. Acad. Sci. USA 102 (2005), 5304-5305.

[LL] J. Lepowsky and H. Li, Introduction to vertex operator algebras and their representations, Progress in Mathematics, Vol. 227, 2004 Birkhüser, Boston.

[Li] H. Li, Some finiteness properties of regular vertex operator algebras, J. Alg. 212 (1999), 495-514. 
[M] M. Miyamoto, Intertwining operators and modular invariance, to appear; math.QA/0010180.

[MS1] G. Moore and N. Seiberg, Polynomial equations for rational conformal field theories, Phys. Lett. B 212 (1988), 451-460.

[MS2] G. Moore and N. Seiberg, Classical and quantum conformal field theory, Comm. Math. Phys. 123 (1989), 177-254.

[S1] G. Segal, The definition of conformal field theory, in: Differential geometrical methods in theoretical physics (Como, 1987), NATO Adv. Sci. Inst. Ser. C Math. Phys. Sci., 250, Kluwer Acad. Publ., Dordrecht, 1988, 165-171.

[S2] G. B. Segal, Two-dimensional conformal field theories and modular functors, in: Proceedings of the IXth International Congress on Mathematical Physics, Swansea, 1988, Hilger, Bristol, 1989, 2237.

[S3] G. Segal, The definition of conformal field theory, preprint, 1988; also in: Topology, geometry and quantum field theory, ed. U. Tillmann, London Math. Soc. Lect. Note Ser., Vol. 308. Cambridge University Press, Cambridge, 2004, 421-577.

[TUY] A. Tsuchiya, K. Ueno and Y. Yamada, Conformal field theory on universal family of stable curves with gauge symmetries, in: $A d$ vanced Studies in Pure Math., Vol. 19, Kinokuniya Company Ltd., Tokyo, 1989, 459-565.

[V] E. Verlinde, Fusion rules and modular transformations in 2D conformal field theory, Nucl. Phys. B300 (1988), 360-376.

[Z] Y. Zhu, Modular invariance of characters of vertex operator algebras, J. Amer. Math. Soc. 9 (1996), 237-307.

Max Planck Institute of Mathematics, Vivatsgasse 7, D-53111 Bonn, GERMANY

Institute of Mathematics, Fudan University, Shanghai, China 
Department of Mathematics, Rutgers University, 110 Frelinghuysen RD., Piscataway, NJ 08854-8019 (PERManent ADDRESS)

E-mail address: yzhuang@math.rutgers.edu 